Supporting Information for

\title{
Pd-Catalyzed Etherification of Nitroarenes
}

Naoki Matsushita, Myuto Kashihara, Michele Formica, and Yoshiaki Nakao*

Department of Material Chemistry, Graduate School of Engineering, Kyoto University, Kyoto 6158510, Japan. 
1. General, Apparatus, and Chemicals S2

$\begin{array}{ll}\text { 2. Ligand synthesis } & \text { S3 }\end{array}$

$\begin{array}{ll}\text { 3. Substrate scope studies } & \text { S6 }\end{array}$

4. Stoichiometric reactions $\quad$ S11

5. X-ray analysis $\quad$ S15

$\begin{array}{ll}\text { 6. Computational details } & \text { S21 }\end{array}$

7. NMR spectra $\quad$ S22

$\begin{array}{ll}\text { 8. References } & \text { S50 }\end{array}$ 


\section{General, Apparatus, and Chemicals}

General. All manipulations of oxygen- and moisture-sensitive materials were conducted with a standard Schlenk technique under an argon atmosphere or in a glove box under a nitrogen atmosphere. Medium pressure liquid chromatography (MPLC) was performed using Kanto Chemical silica gel 60 (spherical, 40-50 $\mu \mathrm{m}$ ) or Biotage ${ }^{\circledR}$ SNAP Ultra. Analytical thin layer chromatography (TLC) was performed on Merck TLC silica gel $60 \mathrm{~F}_{254}(0.25 \mathrm{~mm})$ plates. Visualization was accomplished with UV light (254 nm).

Apparatus. Proton, carbon, fluorine, and phosphorus nuclear magnetic resonance spectra $\left({ }^{1} \mathrm{H}\right.$, ${ }^{13} \mathrm{C},{ }^{19} \mathrm{~F}$, and ${ }^{31} \mathrm{P}$ NMR) were recorded on a JEOL ECS-400 $\left({ }^{1} \mathrm{H}\right.$ NMR, $400 \mathrm{MHz} ;{ }^{13} \mathrm{C}$ NMR, 101 $\mathrm{MHz} ;{ }^{19} \mathrm{~F}$ NMR, $376 \mathrm{MHz} ;{ }^{31} \mathrm{P} \mathrm{NMR}, 162 \mathrm{MHz}$ ) spectrometer with solvent resonance as the internal standard $\left({ }^{1} \mathrm{H} \mathrm{NMR}, \mathrm{CDCl}_{3}\right.$ at $7.26 \mathrm{ppm}, 1$,4-dioxane- $d_{8}$ at $3.50 ;{ }^{13} \mathrm{C} \mathrm{NMR} \mathrm{CDCl}_{3}$ at $77.0 \mathrm{ppm}, \mathrm{C}_{6} \mathrm{D}_{6}$ at $128.0 \mathrm{ppm})$. NMR data are reported as follows: chemical shift, multiplicity ( $\mathrm{s}=$ singlet, $\mathrm{d}=$ doublet, $\mathrm{t}=$ triplet, $\mathrm{q}=$ quartet, quint $=$ quintet, hept $=$ heptet, $\mathrm{m}=$ multiplet $)$, coupling constants $(\mathrm{Hz})$, and integration. High-resolution mass spectra were obtained with Thermo Fisher Scientific MS: Exactive Plus HPLC: UltiMate 3000 (ESI). Medium pressure liquid chromatography (MPLC) was performed with a Yamazen EPLC-W-Prep 2XY or SHOKO SCIENTIFIC Purif-espoir2. Gas chromatography (GC) analyses were performed on a Shimadzu GC-2014 equipped with a BP1 column (SGE Analytical Science, $0.25 \mathrm{~mm}$ x $30 \mathrm{~m}$, pressure $=149.0 \mathrm{kPa}$, detector $=\mathrm{FID}, 290^{\circ} \mathrm{C}$ ) with helium gas as a carrier. Elemental analyses were performed on a J-Science Micro corder JM10, JM11.

Chemicals. Unless otherwise noted, commercially available chemicals were distilled over $\mathrm{CaH}_{2}$ and degassed before use. When commercially available chemicals were solids, they were used without purification. Anhydrous hexane, toluene, tetrahydrofuran (THF) were purchased from Kanto Chemical and purified by passage through activated alumina under positive argon pressure as described by Grubbs et al. ${ }^{1}$ Superdehydrated 1,4-dioxane and chloroform were purchased from FUJIFILM Wako Pure Chemical, stored in a glovebox, and used without further purification. All the other commercially available reagents were purchased from common sources (e.g. Tokyo Chemical Industry, FUJIFILM Wako Pure Chemical, Sigma-Aldrich, Alfa-Aesar, Nacalai Tesque, etc.). 


\section{Ligand synthesis}

General procedure for the ligand synthesis. L3-L7 were synthesized according to the reported procedures. ${ }^{2,3}$

Scheme S1. Synthetic procedures of ligands.

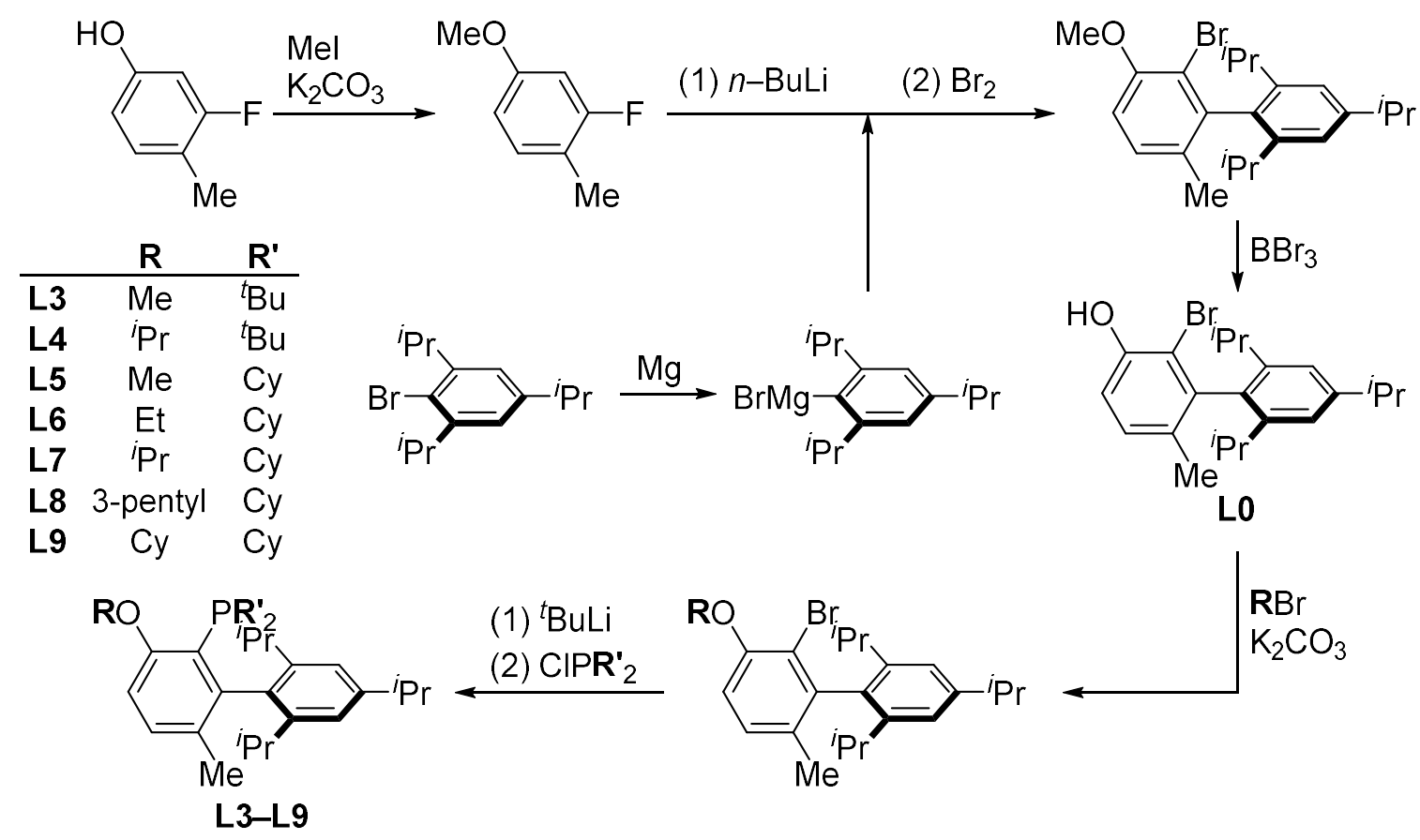

\section{2-Bromo-2',4',6'-triisopropyl-6-methyl-3-(3-pentyloxy)-1,1'-biphenyl.}

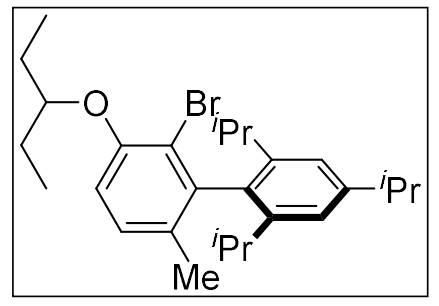

A $20 \mathrm{~mL}$ Schlenk flask was charged with a magnetic stir bar, 2-bromo-3hydroxy-2',4',6'-triisopropyl-6-methy-1,1'-biphenyl (LO) (0.58 g, 1.5 $\mathrm{mmol})$, and $\mathrm{K}_{2} \mathrm{CO}_{3}(0.66 \mathrm{~g}, 4.5 \mathrm{mmol})$. The flask was evacuated and backfilled with argon three times. DMF (3.0 ml) and 3-bomopentane (1.1 $\mathrm{mL}, 9.0 \mathrm{mmol}$ ) were added via syringe and the reaction mixture was stirred overnight at $100{ }^{\circ} \mathrm{C}$. After cooling to room temperature, $\mathrm{H}_{2} \mathrm{O}(20 \mathrm{~mL})$ was added and the reaction mixture was extracted with $\mathrm{Et}_{2} \mathrm{O}(20 \mathrm{~mL}$, three times). The combined organic layer was washed with brine $(50 \mathrm{~mL})$, dried over anhydrous $\mathrm{MgSO}_{4}$, filtered, and evaporated under reduced pressure. The crude product was purified by MPLC [1.5 g Kanto Chemical silica gel 60, $n$-hexane:EtOAc $=97: 3$ ] to afford the title compound $(0.50 \mathrm{~g}, 1.1 \mathrm{mmol}, 72 \%)$ as a colorless solid. $\mathrm{R}_{\mathrm{f}} 0.30$ ( $n$-hexane:EtOAc = 97:3). mp. 162.4-162.8 ${ }^{\circ} \mathrm{C} .{ }^{1} \mathrm{H}$ NMR (400 MHz, $\left.\mathrm{CDCl}_{3}\right): \delta 7.14(\mathrm{~d}, J=8.3 \mathrm{~Hz}, 1 \mathrm{H}), 7.09$ (s, 2H), $6.85(\mathrm{~d}, J=8.3 \mathrm{~Hz}, 1 \mathrm{H}), 4.20$ (quint, $J=4.6 \mathrm{~Hz}, 1 \mathrm{H}$ ), 2.98 (sept, $J=6.9 \mathrm{~Hz}, 1 \mathrm{H}$ ), 2.41 (sept, $J=6.9$ $\mathrm{Hz}, 2 \mathrm{H}), 1.94$ (s, 3H), 1.77 (quint, $J=7.3 \mathrm{~Hz}, 4 \mathrm{H}), 1.33$ (d, $J=6.9 \mathrm{~Hz}, 6 \mathrm{H}), 1.17$ (d, $J=7.0 \mathrm{~Hz}, 6 \mathrm{H}$ ), $1.09(\mathrm{~d}, J=6.9 \mathrm{~Hz}, 6 \mathrm{H}), 1.02(\mathrm{t}, J=7.5 \mathrm{~Hz}, 6 \mathrm{H}) .{ }^{13} \mathrm{C} \mathrm{NMR}\left(101 \mathrm{MHz}, \mathrm{CDCl}_{3}\right): \delta 153.6,148.1,145.4$, 142.5, 135.0, 130.7, 128.7, 120.9, 117.2, 113.9, 82.8, 34.1, 30.6, 26.1, 24.4, 24.3, 24.1, 20.6, 9.7. HRMS-ESI (+) (m/z): [M+Na] $]^{+}$calcd for $\mathrm{C}_{27} \mathrm{H}_{39} \mathrm{BrONa}, 481.2076$; found, 481.2099 . 
(L8).

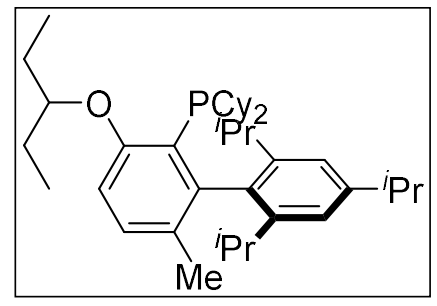

A $20 \mathrm{ml}$ Schlenk flask was charged with a magnetic stir bar and 2-bromo2',4',6'-triisopropyl-6-methyl-3-(3-pentyloxy)-1,1'-biphenyl $\quad(0.23 \mathrm{~g}$, $0.50 \mathrm{mmol})$. The flask was evacuated and backfilled with argon three times, and anhydrous THF $(2.0 \mathrm{~mL})$ was added via syringe. After the flask was cooled to $-78^{\circ} \mathrm{C}$ (acetone/dry ice), $t$-BuLi (1.5 M in pentane, $0.70 \mathrm{ml}, 1.1$ mmol) was added slowly and the reaction mixture was stirred for $30 \mathrm{~min}$ at $-78^{\circ} \mathrm{C}$. The solution was added with $\mathrm{ClPCy}_{2}(0.12 \mathrm{~mL}, 0.55 \mathrm{mmol})$ slowly, warmed to room temperature, and then stirred for $4 \mathrm{~h}$. The crude mixture was filtered through a short pad of Celite ${ }^{\circledR}$ and the filtrate was evaporated under reduced pressure. The obtained solid was recrystallized using hot acetone to afford the title compound (0.26 g, $4.5 \mathrm{mmol}, 90 \%)$ as a colorless solid. mp. 166.9-167.3 ${ }^{\circ} \mathrm{C} .{ }^{1} \mathrm{H} \mathrm{NMR}(400 \mathrm{MHz}$, $\left.\mathrm{CDCl}_{3}\right): \delta 7.14(\mathrm{~d}, J=8.4 \mathrm{~Hz}, 1 \mathrm{H}), 6.98(\mathrm{~s}, 2 \mathrm{H}), 6.68(\mathrm{~d}, J=8.6 \mathrm{~Hz}, 1 \mathrm{H}), 4.20$ (quint, $J=6.0 \mathrm{~Hz}$, 1H), 2.92 (hept, $J=7.5 \mathrm{~Hz}, 1 \mathrm{H}), 2.45$ (hept, $J=6.7 \mathrm{~Hz}, 2 \mathrm{H}), 2.35-2.28$ (m, 2H), 1.83 (q, $J=7.1 \mathrm{~Hz}$, $4 \mathrm{H}), 1.75-1.69(\mathrm{~m}, 7 \mathrm{H}), 1.65-1.58(\mathrm{~m}, 4 \mathrm{H}), 1.46(\mathrm{~d}, J=13.2 \mathrm{~Hz}, 2 \mathrm{H}), 1.29(\mathrm{~d}, J=6.4 \mathrm{~Hz}, 6 \mathrm{H}), 1.26-$ $1.10(\mathrm{~m}, 13 \mathrm{H}), 1.10-1.01(\mathrm{~m}, 7 \mathrm{H}), 1.00-0.93(\mathrm{~m}, 8 \mathrm{H}) .{ }^{13} \mathrm{C} \mathrm{NMR}\left(101 \mathrm{MHz}, \mathrm{CDCl}_{3}\right): \delta 158.7(\mathrm{~d}, J=$ $2.0 \mathrm{~Hz}), 150.4(\mathrm{~d}, J=36.7 \mathrm{~Hz}), 147.0,145.1,136.4(\mathrm{~d}, J=8.5 \mathrm{~Hz}), 131.7,129.4$ (d, $J=7.8 \mathrm{~Hz})$, $123.7(\mathrm{~d}, J=26.8 \mathrm{~Hz}), 120.8,108.9,78.8,37.6$, 37.5, 33.9, 33.6, 33.3, 30.1, 30.1, 27.8, 27.7, 27.7, 27.5, 26.4, 25.4, 25.1, 24.9, 24.0, 21.0, 10.2. ${ }^{31} \mathrm{P}$ NMR (162 MHz, $\left.\mathrm{CDCl}_{3}\right): \delta-2.0$. HRMS-ESI (+) $(\mathrm{m} / \mathrm{z}):[\mathrm{M}+\mathrm{Na}]^{+}$calcd for $\mathrm{C}_{39} \mathrm{H}_{61} \mathrm{OPNa}, 599.4352$; found, 599.4371 .

\section{2-Bromo-3-cyclohexyloxy-2',4',6'-triisopropyl-6-methyl-1,1'-biphenyl.}

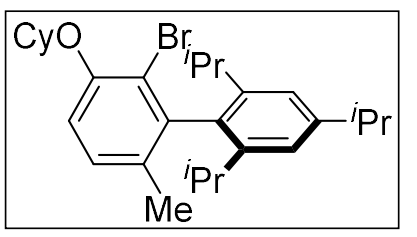

A $80 \mathrm{~mL}$ Schlenk flask was charged with a magnetic stir bar, 2-bromo-3hydroxy-2',4',6'-triisopropyl-6-methy-1,1'-biphenyl (L0) (3.5 g, 9.1 $\mathrm{mmol})$, and $\mathrm{K}_{2} \mathrm{CO}_{3}(3.7 \mathrm{~g}, 27 \mathrm{mmol})$. The flask was evacuated and backfilled with argon three times. DMF $(25 \mathrm{ml})$ and bromocyclohexane $(6.7 \mathrm{~mL}, 55 \mathrm{mmol})$ were added via syringe and the reaction mixture was stirred overnight at $100{ }^{\circ} \mathrm{C}$. After cooling to room temperature, $\mathrm{H}_{2} \mathrm{O}(100 \mathrm{~mL})$ was added and the reaction mixture was extracted with $\mathrm{Et}_{2} \mathrm{O}$ (50 mL, three times). The combined organic layer was washed with brine $(100 \mathrm{~mL})$, dried over anhydrous $\mathrm{MgSO}_{4}$, filtered, and evaporated under reduced pressure. The crude product was purified by MPLC [10 g Kanto Chemical silica gel 60, $n$-hexane:EtOAc $=99: 1$ to 97:3] to afford the title compound $(1.4 \mathrm{~g}, 3.0 \mathrm{mmol}, 33 \%)$ as a colorless solid. $\mathrm{R}_{\mathrm{f}} 0.35$ ( $n$-hexane:EtOAc $\left.=97: 3\right)$. mp. 98.4-98.6 ${ }^{\circ} \mathrm{C} .{ }^{1} \mathrm{H}$ NMR (400 MHz, $\mathrm{CDCl}_{3}$ ): $\delta 7.13(\mathrm{~d}, J=8.2 \mathrm{~Hz}, 1 \mathrm{H}), 7.08(\mathrm{~s}, 2 \mathrm{H}), 6.88(\mathrm{~d}, J=8.2$ $\mathrm{Hz}, 1 \mathrm{H}$ ), 4.32 (quint, $J=4.4 \mathrm{~Hz}, 1 \mathrm{H}$ ), 2.97 (hept, $J=7.1 \mathrm{~Hz}, 1 \mathrm{H}$ ), 2.39 (hept, $J=7.0 \mathrm{~Hz}, 2 \mathrm{H}$ ), 2.07$1.79(\mathrm{~m}, 7 \mathrm{H}), 1.75-1.67(\mathrm{~m}, 2 \mathrm{H}), 1.45-1.25(\mathrm{~m}, 10 \mathrm{H}), 1.16(\mathrm{~d}, J=6.9 \mathrm{~Hz}, 6 \mathrm{H}), 1.08(\mathrm{~d}, J=6.9 \mathrm{~Hz}$, $6 \mathrm{H}) .{ }^{13} \mathrm{C} \mathrm{NMR}\left(101 \mathrm{MHz}, \mathrm{CDCl}_{3}\right): \delta 152.6,148.1,145.4,142.5,135.0,131.1,128.7,120.9,117.6$, 114.8, 77.6, 34.1, 31.7, 30.6, 25.6, 24.4, 24.3, 24.1, 23.5, 20.6. HRMS-ESI $(+)(\mathrm{m} / \mathrm{z}):[\mathrm{M}+\mathrm{Na}]^{+}$calcd for $\mathrm{C}_{28} \mathrm{H}_{39} \mathrm{BrONa}, 493.2076$; found, 493.2098. 


\section{Di-cyclohexyl-(3-(cyclohexyloxy)-2',4',6'-triisopropyl-6-methyl-[1,1'-biphenyl]-2-yl)phosphine}

(L9).

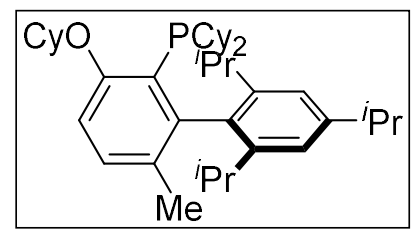

A $80 \mathrm{ml}$ Schlenk flask was charged with a magnetic stir bar and 2-bromo3-cyclohexyloxy-2',4',6'-triisopropyl-6-methyl-1,1'-biphenyl (0.83 g, 1.8 mmol). The flask was evacuated and backfilled with argon three times, and anhydrous THF (15 mL) was added via syringe. After the flask was cooled to $-78^{\circ} \mathrm{C}$ (acetone/dry ice), $t$ - $\mathrm{BuLi}(1.61 \mathrm{M}$ in pentane, $2.2 \mathrm{ml}, 3.5 \mathrm{mmol})$ was added slowly and the reaction mixture was stirred for $50 \mathrm{~min}$ at $-78^{\circ} \mathrm{C}$. The solution was added with $\mathrm{ClPCy}_{2}(0.47 \mathrm{~mL}, 2.1$ mmol) slowly, warmed to room temperature, and then stirred for 2 days at $80{ }^{\circ} \mathrm{C}$. The crude mixture was filtered through a short pad of Celite ${ }^{\circledR}$ and the filtrate was evaporated under reduced pressure. The obtained solid was recrystallized using hot acetone to afford the title compound $(0.67 \mathrm{~g}, 1.1 \mathrm{mmol}$, $65 \%$ ) as a colorless solid. mp. $183.9-184.4{ }^{\circ} \mathrm{C} .{ }^{1} \mathrm{H}$ NMR $\left(400 \mathrm{MHz}, \mathrm{CDCl}_{3}\right): \delta 7.14(\mathrm{~d}, J=8.2 \mathrm{~Hz}$, 1H), 6.99 (s, 2H), 6.72 (d, $J=8.7 \mathrm{~Hz}, 1 \mathrm{H}), 4.30$ (quint, $J=5.0 \mathrm{~Hz}, 1 \mathrm{H}$ ), 2.93 (hept, $J=7.1 \mathrm{~Hz}, 1 \mathrm{H}$ ), 2.45 (hept, $J=7.1 \mathrm{~Hz}, 2 \mathrm{H}), 2.38-2.24$ (m, 2H), 2.20-2.12 (m, 2H), 2.00-1.76 (m, 4H), 1.79-1.52 (m, $11 \mathrm{H}), 1.52-1.08(\mathrm{~m}, 26 \mathrm{H}), 0.98(\mathrm{~d}, J=6.9 \mathrm{~Hz}, 8 \mathrm{H}) .{ }^{13} \mathrm{C} \mathrm{NMR}\left(101 \mathrm{MHz}, \mathrm{CDCl}_{3}\right): \delta 158.2(\mathrm{~d}, J=2.9$ Hz), 150.2 (d, $J=35.9 \mathrm{~Hz}), 147.0,145.1,136.4$ (d, $J=8.1 \mathrm{~Hz}), 131.7,129.3$ (d, $J=7.2 \mathrm{~Hz}), 123.5$ $(\mathrm{d}, J=26.4 \mathrm{~Hz}), 120.8,108.5,74.4,38.2,38.1,33.9,33.6,33.4,32.1,30.2,30.1,30.0,28.1,28.0$, 27.7, 27.6, 26.4 25.7, 25.0, 24.9, 24.3, 24.0, 21.0. ${ }^{31} \mathrm{P}$ NMR (162 MHz, $\left.\mathrm{CDCl}_{3}\right)$ : $\delta-2.7$. HRMS-ESI $(+)(\mathrm{m} / \mathrm{z}):[\mathrm{M}+\mathrm{H}]^{+}$calcd for $\mathrm{C}_{40} \mathrm{H}_{62} \mathrm{OP}, 589.4533$; found, 589.4554 . 


\section{Substrate scope studies}

General procedure for substrate scope. A $15 \mathrm{~mL}$ vial was charged with nitroarene $(0.90 \mathrm{mmol})$, arenol (0.60 mmol), Pd(acac) $2(9.1 \mathrm{mg}, 0.030 \mathrm{mmol}), \mathbf{L 9}(71 \mathrm{mg}, 0.120 \mathrm{mmol})$, and a magnetic stir bar. In a glovebox, $\mathrm{K}_{3} \mathrm{PO}_{4}(382 \mathrm{mg}, 1.8 \mathrm{mmol})$ and 1,4-dioxane $(6.0 \mathrm{~mL})$ were added to the vial (liquid nitroarenes and arenols were added in a glove box). The resulting mixture was taken outside and stirred for $24 \mathrm{~h}$ at $160{ }^{\circ} \mathrm{C}$. After completion of the reaction, the mixture was filtered through a short pad of Celite ${ }^{\circledR}$. The filtrate was added with $\mathrm{H}_{2} \mathrm{O}_{2}\left(30 \mathrm{wt} \%\right.$ in $\left.\mathrm{H}_{2} \mathrm{O}, 5 \mathrm{~mL}\right)$ and $\mathrm{H}_{2} \mathrm{O}(20 \mathrm{~mL})$, and stirred for $5 \mathrm{~min}$ at room temperature. The organic layer was separated. The remained aqueous layer was washed with EtOAc $(10 \mathrm{~mL})$ three times and the combined organic layer was dried over anhydrous $\mathrm{MgSO}_{4}$, and filtered. All volatiles were removed in vacuo and the residue was purified by MPLC using Biotage ${ }^{\circledR}$ SNAP Ultra to afford the corresponding product.

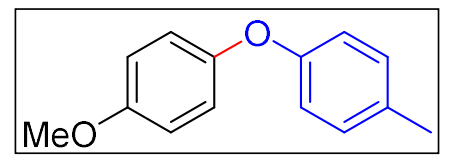

1-Methoxy-4-(p-tolyloxy)benzene (3b). The reaction of 4-nitroanisole (1a) $(0.14 \mathrm{~g}, 0.90 \mathrm{mmol})$ and $p$-cresol (2b) $(65 \mathrm{mg}, 0.60 \mathrm{mmol})$ followed by purification by MPLC ( $n$-hexane/EtOAc $=100: 0$ to $95: 5)$ afforded the title compound (0.12 g, $0.54 \mathrm{mmol}, 90 \%)$ as a colorless solid. ${ }^{1} \mathrm{H} \mathrm{NMR}\left(400 \mathrm{MHz}, \mathrm{CDCl}_{3}\right): \delta 7.10(\mathrm{~d}$, $J=7.7 \mathrm{~Hz}, 2 \mathrm{H}), 6.95(\mathrm{~d}, J=9.3 \mathrm{~Hz}, 2 \mathrm{H}), 6.90-6.81(\mathrm{~m}, 4 \mathrm{H}), 3.80(\mathrm{~s}, 3 \mathrm{H}), 2.31(\mathrm{~s}, 3 \mathrm{H}) .{ }^{13} \mathrm{C} \mathrm{NMR}$ $\left(101 \mathrm{MHz}, \mathrm{CDCl}_{3}\right): \delta 156.1,155.6,150.7,132.0,130.1,120.3,117.8,114.8,55.6,20.6$. All resonances of ${ }^{1} \mathrm{H}$ and ${ }^{13} \mathrm{C}$ NMR spectra were consistent with the reported values. ${ }^{4}$

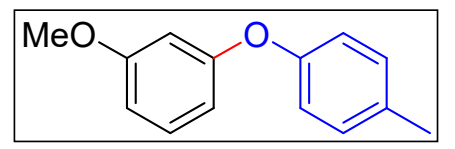

1-Methoxy-3-(p-tolyloxy)benzene (3c). The reaction of 3-nitroanisole (1b) $(0.14 \mathrm{~g}, 0.90 \mathrm{mmol})$ and $p$-cresol (2b) $(65 \mathrm{mg}, 0.60 \mathrm{mmol})$ followed by purification by MPLC ( $n$-hexane/EtOAc $=97: 3$ to $90: 10)$ afforded the title compound (99 mg, $0.46 \mathrm{mmol}, 77 \%$ ) as a colorless oil. ${ }^{1} \mathrm{H}$ NMR (400 MHz, $\left.\mathrm{CDCl}_{3}\right): \delta 7.21(\mathrm{t}, J$ $=8.4 \mathrm{~Hz}, 1 \mathrm{H}), 7.15(\mathrm{~d}, J=7.8 \mathrm{~Hz}, 2 \mathrm{H}), 6.95(\mathrm{~d}, J=7.3 \mathrm{~Hz}, 2 \mathrm{H}), 6.64(\mathrm{~d}, J=8.3 \mathrm{~Hz}, 1 \mathrm{H}), 6.60-6.54$ (m, 2H), 3.78 (s, 3H), 2.35 (s, 3H). $\left.{ }^{13} \mathrm{C} \mathrm{NMR} \mathrm{(101} \mathrm{MHz,} \mathrm{CDCl}_{3}\right): \delta 160.9,159.1,154.4,133.0,130.2$, 130.0, 119.3, 110.4, 108.4, 104.3, 55.3, 20.7. All resonances of ${ }^{1} \mathrm{H}$ and ${ }^{13} \mathrm{C}$ NMR spectra were consistent with the reported values. ${ }^{5}$

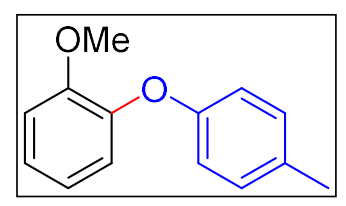

1-Methoxy-2-(p-tolyloxy)benzene (3d). The reaction of 2-nitroanisole (1c) $(0.14 \mathrm{~g}, 0.90 \mathrm{mmol})$ and $p$-cresol (2b) $(65 \mathrm{mg}, 0.60 \mathrm{mmol})$ followed by purification by MPLC ( $n$-hexane/EtOAc $=97: 3$ to $90: 10$ ) afforded the title compound (92 mg, $0.43 \mathrm{mmol}, 72 \%$ ) as a colorless solid. ${ }^{1} \mathrm{H}$ NMR (400 MHz, $\left.\mathrm{CDCl}_{3}\right): \delta 7.10(\mathrm{~d}, J=8.0 \mathrm{~Hz}, 3 \mathrm{H}), 7.00(\mathrm{~d}, J=8.0 \mathrm{~Hz}, 1 \mathrm{H}), 6.97-6.84(\mathrm{~m}, 4 \mathrm{H}), 3.86(\mathrm{~s}, 3 \mathrm{H}), 2.32(\mathrm{~s}$, $3 \mathrm{H}) .{ }^{13} \mathrm{C} \mathrm{NMR}\left(101 \mathrm{MHz}, \mathrm{CDCl}_{3}\right): \delta 155.4,151.2,145.7,132.0,130.0,124.3,121.0,120.3,117.5$, 112.7, 56.0, 20.6. All resonances of ${ }^{1} \mathrm{H}$ and ${ }^{13} \mathrm{C}$ NMR spectra were consistent with the reported values. $^{6}$ 


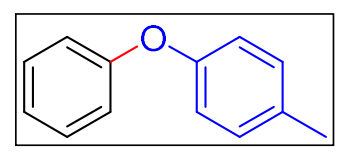

1-Methyl-4-phenoxybenzene (3e). The reaction of nitrobenzene (1d) $(92 \mu \mathrm{L}$, $0.90 \mathrm{mmol})$ and $p$-cresol (2b) $(65 \mathrm{mg}, 0.60 \mathrm{mmol})$ followed by purification by MPLC $(n$-hexane/EtOAc $=97: 3)$ afforded the title compound $(96 \mathrm{mg}, 0.52$ mmol, 87\%) as a colorless oil. ${ }^{1} \mathrm{H}$ NMR $\left(400 \mathrm{MHz}^{\mathrm{CDCl}} 3\right): \delta 7.32(\mathrm{t}, J=7.8 \mathrm{~Hz}, 2 \mathrm{H}), 7.15(\mathrm{~d}, J=$ $8.0 \mathrm{~Hz}, 2 \mathrm{H}), 7.08$ (t, $J=7.3 \mathrm{~Hz}, 1 \mathrm{H}), 6.99$ (d, $J=8.1 \mathrm{~Hz}, 2 \mathrm{H}), 6.93$ (d, $J=7.9 \mathrm{~Hz}, 2 \mathrm{H}), 2.35$ (s, 3H). ${ }^{13} \mathrm{C}$ NMR (101 MHz, $\left.\mathrm{CDCl}_{3}\right): \delta 157.8,154.7,132.9,130.2,129.6,122.8,119.1,118.3,20.7$. All resonances of ${ }^{1} \mathrm{H}$ and ${ }^{13} \mathrm{C}$ NMR spectra were consistent with the reported values. ${ }^{7}$

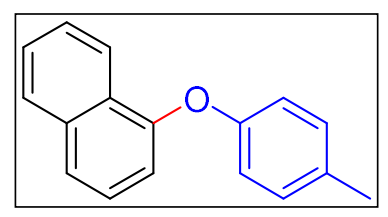

1-(p-Tolyloxy)naphthalene (3f). The reaction of 1-nitronaphthalene (1e) $(0.16 \mathrm{~g}, 0.90 \mathrm{mmol})$ and $p$-cresol $(\mathbf{2 b})(65 \mathrm{mg}, 0.60 \mathrm{mmol})$ followed by purification by MPLC $(n$-hexane/EtOAc $=97: 3)$ afforded the title compound (0.13 g, $0.53 \mathrm{mmol}, 89 \%$ ) as a colorless solid. ${ }^{1} \mathrm{H}$ NMR (400 $\left.\mathrm{MHz}, \mathrm{CDCl}_{3}\right)$ : $\delta 8.37(\mathrm{~d}, J=7.6 \mathrm{~Hz}, 1 \mathrm{H}), 7.95(\mathrm{~d}, J=7.5 \mathrm{~Hz}, 1 \mathrm{H}), 7.67(\mathrm{~d}, J=8.2 \mathrm{~Hz}, 1 \mathrm{H}), 7.64-7.54(\mathrm{~m}, 2 \mathrm{H}), 7.44$ (t, $J=8.0 \mathrm{~Hz}, 1 \mathrm{H}), 7.24(\mathrm{~d}, J=7.9 \mathrm{~Hz}, 2 \mathrm{H}), 7.07$ (d, $J=7.8 \mathrm{~Hz}, 2 \mathrm{H}), 7.00(\mathrm{~d}, J=7.5 \mathrm{~Hz}, 1 \mathrm{H}), 2.43$ (s, 3H). ${ }^{13} \mathrm{C}$ NMR $\left(101 \mathrm{MHz}, \mathrm{CDCl}_{3}\right): \delta 155.3,153.6,134.9,132.8,130.3,127.7,126.7,126.5,125.8$, $125.7,122.8,122.1,118.8,112.5,20.7$. All resonances of ${ }^{1} \mathrm{H}$ and ${ }^{13} \mathrm{C}$ NMR spectra were consistent with the reported values. ${ }^{8}$

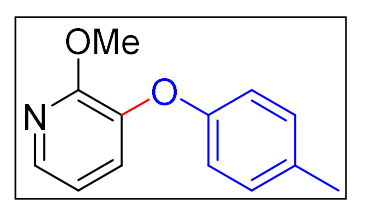

2-Methoxy-3-(p-tolyloxy)pyridine (3g). The reaction of 2-methoxy-3nitropyridine (1f) (0.14 g, $0.90 \mathrm{mmol})$ and $p$-cresol (2b) $(65 \mathrm{mg}, 0.60 \mathrm{mmol})$ followed by purification by MPLC $(n$-hexane/EtOAc $=97: 3)$ afforded the title compound (86 mg, $0.40 \mathrm{mmol}, 67 \%)$ as a colorless solid. $\mathrm{R}_{\mathrm{f}} 0.40$ ( $n$ hexane:EtOAc =97:3). mp. 50.1-50.7 ${ }^{\circ} \mathrm{C} .{ }^{1} \mathrm{H} \mathrm{NMR}\left(400 \mathrm{MHz}, \mathrm{CDCl}_{3}\right): \delta 7.90(\mathrm{~d}, J=4.8 \mathrm{~Hz}, 1 \mathrm{H})$, $7.13(\mathrm{~d}, J=8.0 \mathrm{~Hz}, 2 \mathrm{H}), 7.09(\mathrm{~d}, J=7.7 \mathrm{~Hz}, 1 \mathrm{H}), 6.88(\mathrm{~d}, J=8.0 \mathrm{~Hz}, 2 \mathrm{H}), 6.82$ (t, $J=6.2 \mathrm{~Hz}, 1 \mathrm{H})$, $4.01(\mathrm{~s}, 3 \mathrm{H}), 2.33(\mathrm{~s}, 3 \mathrm{H}) .{ }^{13} \mathrm{C} \mathrm{NMR}\left(101 \mathrm{MHz} \mathrm{CDCl}_{3}\right): \delta 156.0,154.1,141.5,140.4,133.2,130.3$, 125.7, 118.3, 116.9, 53.7, 20.7. HRMS-ESI $(+)(\mathrm{m} / \mathrm{z}):[\mathrm{M}+\mathrm{Na}]^{+}$calcd for $\mathrm{C}_{13} \mathrm{H}_{13} \mathrm{NO}_{2} \mathrm{Na}, 238.0838$; found, 238.0847 .

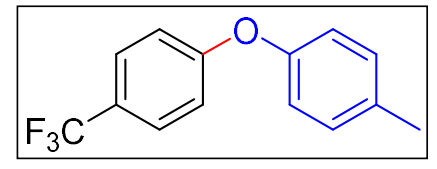

1-Methyl-4-(4-trifluoromethylphenoxy)benzene (3h). The reaction of 4-nitrobenzotrifluoride (1g) $(0.17 \mathrm{~g}, 0.90 \mathrm{mmol})$ and $p$-cresol (2b) $(65 \mathrm{mg}$, $0.60 \mathrm{mmol})$ followed by purification by MPLC $(n$-hexane/EtOAc $=95: 5)$ afforded the title compound ( $80 \mathrm{mg}, 0.32 \mathrm{mmol}, 53 \%)$ as a colorless solid. ${ }^{1} \mathrm{H}$ NMR (400 MHz, $\left.\mathrm{CDCl}_{3}\right): \delta 7.56(\mathrm{~d}, J=8.3 \mathrm{~Hz}, 2 \mathrm{H}), 7.20(\mathrm{~d}, J=8.0 \mathrm{~Hz}, 2 \mathrm{H}), 7.02(\mathrm{~d}, J=8.4 \mathrm{~Hz}, 2 \mathrm{H}), 6.97(\mathrm{~d}, J=8.0$ $\mathrm{Hz}, 2 \mathrm{H}), 2.38(\mathrm{~s}, 3 \mathrm{H}) .{ }^{13} \mathrm{C} \mathrm{NMR}\left(101 \mathrm{MHz}, \mathrm{CDCl}_{3}\right): \delta 161.0,153.2,134.4,130.5,127.0$ (q, $J=4.6$ $\mathrm{Hz}), 124.4$ (q, $J=32.5 \mathrm{~Hz}) 124.2(\mathrm{q}, J=273.0 \mathrm{~Hz}), 120.0,117.3,20.7 .{ }^{19} \mathrm{~F} \mathrm{NMR}\left(376 \mathrm{MHz}, \mathrm{CDCl}_{3}\right)$ : $\delta-62.0$. All resonances of ${ }^{1} \mathrm{H}$ and ${ }^{13} \mathrm{C}$ NMR spectra were consistent with the reported values. ${ }^{9}$

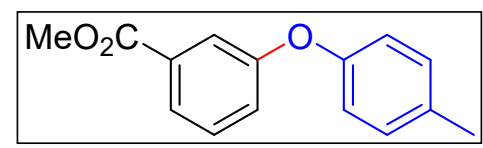

Methyl 3-(p-tolyloxy)benzoate (3i). The reaction of methyl 3nitrobenzoate (1h) $(0.16 \mathrm{~g}, 0.90 \mathrm{mmol})$ and $p$-cresol (2b) $(65 \mathrm{mg}, 0.60$ 
mmol) followed by purification by MPLC $(n$-hexane/EtOAc $=97: 3)$ afforded the title compound (52 $\mathrm{mg}, 0.21 \mathrm{mmol}, 36 \%)$ as a colorless oil. $\mathrm{R}_{\mathrm{f}} 0.30$ ( $n$-hexane:EtOAc $\left.=97: 3\right) .{ }^{1} \mathrm{H}$ NMR $(400 \mathrm{MHz}$, $\left.\mathrm{CDCl}_{3}\right): \delta 7.75(\mathrm{~d}, J=7.6 \mathrm{~Hz}, 1 \mathrm{H}), 7.64(\mathrm{~s}, 1 \mathrm{H}), 7.38(\mathrm{t}, J=7.9 \mathrm{~Hz}, 1 \mathrm{H}), 7.22-7.13(\mathrm{~m}, 3 \mathrm{H}), 6.92(\mathrm{~d}$, $J=6.7 \mathrm{~Hz}, 2 \mathrm{H}), 3.89(\mathrm{~s}, 3 \mathrm{H}), 2.35(\mathrm{~s}, 3 \mathrm{H}) .{ }^{13} \mathrm{C} \mathrm{NMR}\left(101 \mathrm{MHz}, \mathrm{CDCl}_{3}\right): \delta 166.5,158.0,154.1,133.4$, 131.8, 130.4, 129.6, 123.9, 122.8, 119.2, 118.9, 52.2, 20.7. HRMS-ESI (+) (m/z): $[\mathrm{M}+\mathrm{Na}]^{+}$calcd for $\mathrm{C}_{15} \mathrm{H}_{14} \mathrm{O}_{3} \mathrm{Na}$, 265.0835; found, 265.0834.

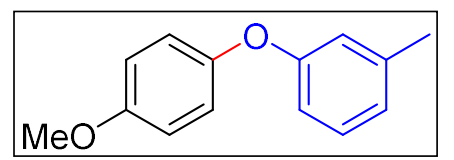

1-(4-Methoxyphenoxy)-3-methylbenzene (3j). The reaction of 4nitroanisole (1a) $(0.14 \mathrm{~g}, 0.90 \mathrm{mmol})$ and $m$-cresol $(\mathbf{2 c})(65 \mathrm{mg}, 0.60$ mmol) followed by purification by MPLC $(n$-hexane/EtOAc $=97: 3)$ afforded the title compound (96 mg, $0.45 \mathrm{mmol}, 74 \%$ ) as a colorless solid. ${ }^{1} \mathrm{H}$ NMR (400 MHz, $\left.\mathrm{CDCl}_{3}\right): \delta 7.18(\mathrm{t}, J=7.7 \mathrm{~Hz}, 1 \mathrm{H}), 6.98(\mathrm{~d}, J=8.6 \mathrm{~Hz}, 2 \mathrm{H}), 6.92-6.83(\mathrm{~m}, 3 \mathrm{H}), 6.75(\mathrm{~d}, 2 \mathrm{H}), 3.81(\mathrm{~s}$, $3 \mathrm{H}), 2.31(\mathrm{~s}, 3 \mathrm{H}) .{ }^{13} \mathrm{C} \mathrm{NMR}\left(101 \mathrm{MHz}, \mathrm{CDCl}_{3}\right): \delta 158.5,155.8,150.2,139.8,129.3,123.2,120.8$, 118.2, 114.8, 114.6, 55.6, 21.4. All resonances of ${ }^{1} \mathrm{H}$ and ${ }^{13} \mathrm{C}$ NMR spectra were consistent with the reported values. ${ }^{10}$

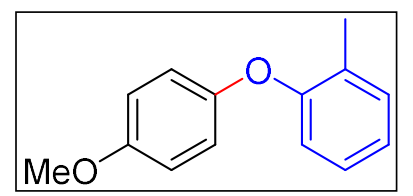

1-(4-Methoxyphenoxy)-2-methylbenzene (3k). The reaction of 4nitroanisole (1a) $(0.14 \mathrm{~g}, 0.90 \mathrm{mmol})$ and $o$-cresol (2d) $(65 \mathrm{mg}, 0.60 \mathrm{mmol})$ followed by purification by MPLC ( $n$-hexane/EtOAc $=97: 3$ to $95: 5$ ) afforded the title compound $(0.10 \mathrm{~g}, 0.49 \mathrm{mmol}, 81 \%)$ as a colorless oil. ${ }^{1} \mathrm{H}$ NMR (400 MHz, $\left.\mathrm{CDCl}_{3}\right): \delta 7.24(\mathrm{~d}, J=7.5 \mathrm{~Hz}, 1 \mathrm{H}), 7.13(\mathrm{t}, J=7.8 \mathrm{~Hz}, 1 \mathrm{H}), 7.02(\mathrm{t}, J=7.4 \mathrm{~Hz}, 1 \mathrm{H})$, 6.94-6.84 (m, 4H), $6.81(\mathrm{~d}, J=8.0 \mathrm{~Hz}, 1 \mathrm{H}), 3.80$ (s, 3H), 2.29 (s, 3H). ${ }^{13} \mathrm{C} \mathrm{NMR}\left(101 \mathrm{MHz}, \mathrm{CDCl}_{3}\right)$ : $\delta 155.8,155.3,151.1,131.3,129.0,126.9,123.0,119.3,118.0,114.8,55.6,16.2$. All resonances of ${ }^{1} \mathrm{H}$ and ${ }^{13} \mathrm{C}$ NMR spectra were consistent with the reported values. ${ }^{7}$

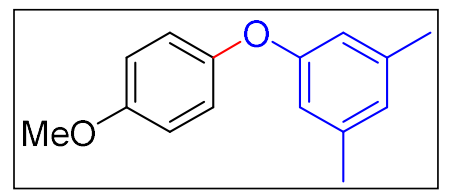

1-(4-Methoxyphenoxy)-3,5-dimethylbenzene (3I). The reaction of 4nitroanisole (1a) (0.14 g, $0.90 \mathrm{mmol})$ and 3,5-dimethylphenol (2e) $(73 \mathrm{mg}$, $0.60 \mathrm{mmol})$ followed by purification by MPLC $(n$-hexane/EtOAc $=97: 3)$ afforded the title compound $(0.10 \mathrm{~g}, 0.46 \mathrm{mmol}, 77 \%)$ as a colorless solid. ${ }^{1} \mathrm{H}$ NMR (400 MHz, $\left.\mathrm{CDCl}_{3}\right): \delta$ 7.01-6.93 (m, 2H), 6.91-6.85 (m, 2H), $6.69(\mathrm{~s}, 1 \mathrm{H}), 6.56(\mathrm{~s}, 2 \mathrm{H})$, $3.81(\mathrm{~s}, 3 \mathrm{H}), 2.27(\mathrm{~s}, 6 \mathrm{H}) .{ }^{13} \mathrm{C}$ NMR $\left(101 \mathrm{MHz}, \mathrm{CDCl}_{3}\right): \delta 158.5,155.7,150.3,139.4,124.2,120.8$, 115.3, 114.8, 55.6, 21.3. All resonances of ${ }^{1} \mathrm{H}$ and ${ }^{13} \mathrm{C}$ NMR spectra were consistent with the reported values. $^{7}$

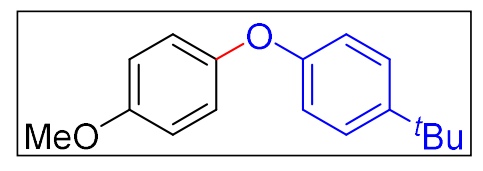

1-(4-tert-Butylphenoxy)-4-methoxybenzene (3m). The reaction of 4nitroanisole (1a) $(0.14 \mathrm{~g}, 0.90 \mathrm{mmol})$ and 4-tert-butylphenol (2f) $(90$ $\mathrm{mg}, 0.60 \mathrm{mmol})$ followed by purification by MPLC $(n$-hexane/EtOAc $=$ 100:0 to 90:10) afforded the title compound ( $0.12 \mathrm{~g}, 0.54 \mathrm{mmol}, 90 \%)$ as a colorless solid. ${ }^{1} \mathrm{H}$ NMR $\left(400 \mathrm{MHz}, \mathrm{CDCl}_{3}\right): \delta 7.31(\mathrm{~d}, J=8.7 \mathrm{~Hz}, 2 \mathrm{H}), 6.98(\mathrm{~d}, J=9.2 \mathrm{~Hz}, 2 \mathrm{H}), 6.88(\mathrm{~d}, J=8.0 \mathrm{~Hz}, 4 \mathrm{H}), 3.80$ 
(s, 3H), 1.31 (s, 9H). $\left.{ }^{13} \mathrm{C} \mathrm{NMR} \mathrm{(101} \mathrm{MHz,} \mathrm{CDCl}_{3}\right): \delta$ 156.0, 155.7, 150.5, 145.2, 126.4, 120.6, 117.1, 114.8, 55.6, 34.2, 31.5. All resonances of ${ }^{1} \mathrm{H}$ and ${ }^{13} \mathrm{C}$ NMR spectra were consistent with the reported values. $^{4}$

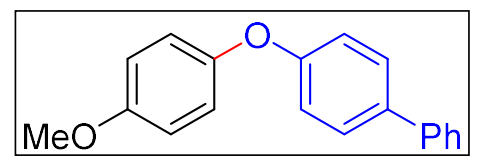

4-(4-Methoxyphenoxy)-1,1'-biphenyl (3n). The reaction of 4nitroanisole (1a) (0.14 g, $0.90 \mathrm{mmol})$ and 4-phenylphenol (2g) $(0.10 \mathrm{mg}$, $0.60 \mathrm{mmol})$ followed by purification by MPLC $(n$-hexane/EtOAc $=97: 3$ to $90: 10)$ afforded the title compound (98 $\mathrm{mg}, 0.35 \mathrm{mmol}, 59 \%)$ as a colorless solid. ${ }^{1} \mathrm{H}$ NMR (400 $\left.\mathrm{MHz} \mathrm{CDCl}_{3}\right): \delta 7.59-7.49(\mathrm{~m}, 4 \mathrm{H}), 7.42(\mathrm{t}, J=7.5 \mathrm{~Hz}, 2 \mathrm{H}), 7.36-7.28(\mathrm{~m}, 1 \mathrm{H}), 7.06-6.98(\mathrm{~m}, 4 \mathrm{H})$, $6.91(\mathrm{~d}, J=8.0 \mathrm{~Hz}, 2 \mathrm{H}), 3.82(\mathrm{~s}, 3 \mathrm{H}) .{ }^{13} \mathrm{C} \mathrm{NMR}\left(101 \mathrm{MHz}, \mathrm{CDCl}_{3}\right): \delta 158.1,156.0,150.0,140.6$, 135.5, 128.7, 128.3, 126.9, 126.8, 120.9, 117.7, 114.9, 77.3, 77.0, 76.7, 55.7. All resonances of ${ }^{1} \mathrm{H}$ and ${ }^{13} \mathrm{C}$ NMR spectra were consistent with the reported values. ${ }^{4}$

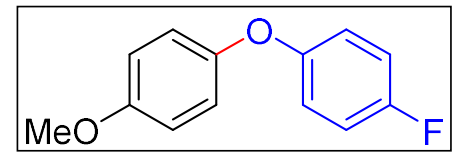

1-Fluoro-4-(4-methoxyphenoxy)benzene (30). The reaction of 4nitroanisole (1a) $(0.14 \mathrm{~g}, 0.90 \mathrm{mmol})$ and 4-fluorophenol (2h) $(67 \mathrm{mg}$, $0.60 \mathrm{mmol})$ followed by purification by MPLC $(n$-hexane/EtOAc $=97: 3)$ afforded the title compound $(61 \mathrm{mg}, 0.28 \mathrm{mmol}, 47 \%)$ as a colorless solid. ${ }^{1} \mathrm{H}$ NMR (400 MHz, $\left.\mathrm{CDCl}_{3}\right): \delta 7.02-6.82(\mathrm{~m}, 8 \mathrm{H}), 3.79(\mathrm{~s}, 3 \mathrm{H}) .{ }^{13} \mathrm{C} \mathrm{NMR}\left(101 \mathrm{MHz}, \mathrm{CDCl}_{3}\right): \delta 158.3(\mathrm{~d}, J=240.4 \mathrm{~Hz})$, 155.8, 154.2 (d, $J=2.0 \mathrm{~Hz}), 150.6,120.2,119.1$ (d, $J=8.1 \mathrm{~Hz}), 116.1$ (d, $J=23.2 \mathrm{~Hz}), 114.9$, 55.6. ${ }^{19} \mathrm{~F}$ NMR (376 MHz, $\mathrm{CDCl}_{3}$ ): $\delta$-121.6. All resonances of ${ }^{1} \mathrm{H},{ }^{13} \mathrm{C}$, and ${ }^{19} \mathrm{~F} \mathrm{NMR} \mathrm{spectra} \mathrm{were}$ consistent with the reported values. ${ }^{7}$

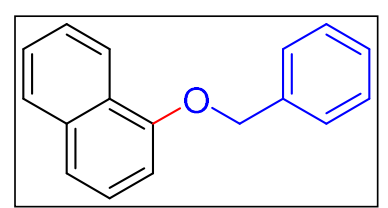

1-(Benzyloxy)naphthalene (3p). The reaction of 1-nitronaphthalene (1e) $(0.10 \mathrm{~g}, 0.60 \mathrm{mmol})$ and benzyl alcohol $(2 \mathbf{i})(93 \mu \mathrm{L}, 0.90 \mathrm{mmol})$ followed by purification by MPLC ( $n$-hexane/EtOAc $=100: 0$ to 95:5) afforded the title compound (39 mg, $0.17 \mathrm{mmol}, 28 \%$ ) as a pale red oil. ${ }^{1} \mathrm{H}$ NMR (400 MHz, $\left.\mathrm{CDCl}_{3}\right): \delta 8.37(\mathrm{~d}, J=7.8 \mathrm{~Hz}, 1 \mathrm{H}), 7.82(\mathrm{~d}, J=8.2 \mathrm{~Hz}, 1 \mathrm{H}), 7.58-7.34(\mathrm{~m}, 9 \mathrm{H}), 6.91(\mathrm{~d}, J=7.8 \mathrm{~Hz}$, 1H), $5.27(\mathrm{~s}, 2 \mathrm{H}) .{ }^{13} \mathrm{C}$ NMR $\left(101 \mathrm{MHz}, \mathrm{CDCl}_{3}\right): \delta 154.5,137.1,134.5,128.6,127.9,127.4,127.4$, $126.4,125.8,125.7,125.2,122.2,120.5,105.2,70.1$. All resonances of ${ }^{1} \mathrm{H}$ and ${ }^{13} \mathrm{C}$ NMR spectra were consistent with the reported values. ${ }^{11}$

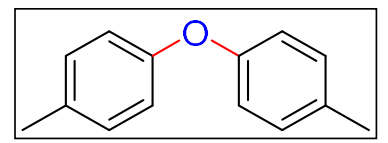

4,4'-Dimethyldiphenyl ether (3q). The reaction of 4-nitrotoluene (1i) (82 $\mathrm{mg}, 0.60 \mathrm{mmol})$ and $\mathrm{H}_{2} \mathrm{O}(16 \mu \mathrm{L}, 0.90 \mathrm{mmol})$ followed by purification by MPLC ( $n$-hexane/EtOAc $=100: 0$ to 95:5) afforded the title compound (32 $\mathrm{mg}, 0.16 \mathrm{mmol}, 54 \%$ ) as a pale red solid. $\left.{ }^{1} \mathrm{H} \mathrm{NMR} \mathrm{(400} \mathrm{MHz,} \mathrm{CDCl}_{3}\right): \delta 7.12(\mathrm{~d}, J=8.2 \mathrm{~Hz}, 4 \mathrm{H})$, 6.89 (d, $J=6.4 \mathrm{~Hz}, 4 \mathrm{H}), 2.33$ (s, 6H). $\left.{ }^{13} \mathrm{C} \mathrm{NMR} \mathrm{(101} \mathrm{MHz,} \mathrm{CDCl}_{3}\right): \delta 155.3,132.4,130.1,118.6$, 20.6. All resonances of ${ }^{1} \mathrm{H}$ and ${ }^{13} \mathrm{C}$ NMR spectra were consistent with the reported values. ${ }^{5}$ 


\section{Nitroarenes}<smiles>[X]c1ccc([N+](=O)[O-])cc1</smiles>

$\mathrm{X}=\mathrm{Br}, \mathrm{Cl}$<smiles>N#Cc1ccc([N+](=O)[O-])cc1</smiles>

3,4 position<smiles>O=[N+]([O-])c1cccc2ncccc12</smiles>

\section{Arenols}

$\mathrm{HO}$<smiles>FC1C=CCCC1</smiles>

3,4 position<smiles>Oc1cnc2ccccc2c1</smiles>

2,4 position<smiles>Nc1ccc([N+](=O)[O-])cc1</smiles><smiles>O=[N+]([O-])c1ccc(O)cc1</smiles><smiles>[R]C(=O)c1ccc([N+](=O)[O-])cc1</smiles>

$\mathrm{R}=\mathrm{H}, \mathrm{OH}, \mathrm{Me}, \mathrm{NH}_{2}$<smiles>O=[N+]([O-])c1ccc(CO)cc1</smiles><smiles>Cc1ccccc1[N+](=O)[O-]</smiles><smiles>O=[N+]([O-])c1cccc2[nH]ccc12</smiles><smiles>O=[N+]([O-])c1cccc2ccncc12</smiles><smiles>O=[N+]([O-])c1cccc2cccnc12</smiles><smiles>Oc1c(F)c(F)c(F)c(F)c1F</smiles><smiles>Cc1cccc(O)n1</smiles><smiles>Cc1cccnc1O</smiles><smiles>CN(C)c1cccc(O)c1</smiles>

Figure S1. Unsuccessful substrates. 


\section{Stoichiometric reactions}

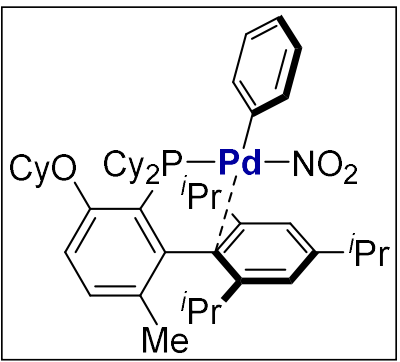

Reaction of (cod)Pd( $\left.\mathrm{CH}_{2} \mathrm{SiMe}_{3}\right)_{2}$ with nitrobenzene (1d) in the presence of L9: Synthesis of (L9)Pd(Ph)(NO charged with $\mathbf{L 9}$ (97 $\mathrm{mg}, 0.25 \mathrm{mmol}$ ) and a magnetic stir bar. In a glovebox, nitrobenzene (1d) $(0.15 \mu \mathrm{L}, 1.5 \mathrm{mmol}),(\mathrm{cod}) \mathrm{Pd}\left(\mathrm{CH}_{2} \mathrm{SiMe}_{3}\right)_{2}(0.14 \mathrm{~g}, 0.25$ $\mathrm{mmol})$ and THF $(8.0 \mathrm{~mL})$ were added to the vial and allowed to stir for 10 min at ambient temperature. The resulting mixture was stirred for $48 \mathrm{~h}$ at $60{ }^{\circ} \mathrm{C}$. After completion of the reaction, removal of the solvent under reduced pressure gave a black solid, which was washed with hexane ( $2 \mathrm{~mL}, 5$ times) to remove unreacted $\mathbf{L 9}$ and $1 \mathrm{~d}$. The residue was extracted with $10 \mathrm{~mL}$ of $\mathrm{CHCl}_{3}$. The obtained solution was evaporated under reduced pressure to afford the title compound as a yellow solid (60 mg, $73 \mu \mathrm{mol}, 29 \%)$. Crystals suitable for X-ray crystallography were obtained by slow diffusion of hexane into a saturated $\mathrm{CHCl}_{3}$ solution at room temperature. mp. $183.0{ }^{\circ} \mathrm{C}$ (dec.). ${ }^{1} \mathrm{H}$ NMR $\left(400 \mathrm{MHz}, \mathrm{CDCl}_{3}\right): \delta 7.26-7.07(\mathrm{~m}, 5 \mathrm{H}), 6.78(\mathrm{~d}, J=7.0$ $\mathrm{Hz}, 4 \mathrm{H}), 4.44-4.28(\mathrm{~m}, 1 \mathrm{H}), 2.96-2.71(\mathrm{~m}, 3 \mathrm{H}), 2.60$ (hept, $J=7.1 \mathrm{~Hz}, 2 \mathrm{H}), 2.19(\mathrm{~d}, J=11.7 \mathrm{~Hz}$, 2H), $1.95(\mathrm{~d}, J=6.6 \mathrm{~Hz}, 5 \mathrm{H}), 1.88(\mathrm{~d}, J=12.8 \mathrm{~Hz}, 2 \mathrm{H}), 1.78(\mathrm{~d}, J=9.5 \mathrm{~Hz}, 2 \mathrm{H}), 1.74-1.63(\mathrm{~m}, 11 \mathrm{H})$, $1.58-1.35(\mathrm{~m}, 5 \mathrm{H}), 1.33-1.04(\mathrm{~m}, 18 \mathrm{H}), 0.96(\mathrm{~d}, J=6.6 \mathrm{~Hz}, 6 \mathrm{H}) .{ }^{13} \mathrm{C} \mathrm{NMR}\left(101 \mathrm{MHz}, \mathrm{CDCl}_{3}\right): \delta$ $157.5,153.9,149.6,148.3,148.1,140.8,136.9$, 134.9, 131.0, 130.9, 126.1 125.0, 123.6, 122.3, 120.8, 111.0, 76.1, 35.8, 35.5, 34.0, 32.0, 31.7, 29.6, 29.2, 27.7, 27.6, 26.6, 26.0, 25.5, 24.6, 24.4, 24.3, 23.0, 19.3. Observed complexity is due to C-P coupling. ${ }^{31} \mathrm{P} \mathrm{NMR}\left(162 \mathrm{MHz}, \mathrm{CDCl}_{3}\right)$ : $\delta 35.5$ (34.7 in 1,4dioxane- $\left.d_{8}\right)$. HRMS-ESI $(+)(\mathrm{m} / \mathrm{z})$ : $\left[\mathrm{M}-\mathrm{NO}_{2}\right]^{+}$calcd for $\mathrm{C}_{46} \mathrm{H}_{66} \mathrm{OPPd}, 771.3881$; found, 771.3894 . Anal. Calcd for $\mathrm{C}_{46} \mathrm{H}_{66} \mathrm{O}_{3} \mathrm{NPPd} \cdot 0.8 \mathrm{CHCl}_{3}: \mathrm{C}, 61.51 ; \mathrm{H}, 7.37 ; \mathrm{N}, 1.53$. Found: $\mathrm{C}, 61.42 ; \mathrm{H}, 7.34 ; \mathrm{N}$, 1.46. Elementral analysis was carried out using crystals of $\mathbf{4 a}$, which were prepared as well as those for X-ray crystallographic analysis. The X-ray analysis showed that the obtained crystals include the equimolar amount of $\mathrm{CHCl}_{3}$ molecule (Fig. S6). It was difficult to remove $\mathrm{CHCl}_{3}$ completely in spite of long-time evacuation. Elemental analysis suggested only $20 \%$ of $\mathrm{CHCl}_{3}$ was removed.

Reaction of ( $\mathrm{L9}) \mathrm{Pd}(\mathrm{Ph})\left(\mathrm{NO}_{2}\right)$ (4a) with $p$-cresol $(2 \mathrm{~b})$ in the presence of $\mathrm{K}_{3} \mathrm{PO}_{4}$ : Synthesis of $(\mathrm{L9}) \mathrm{Pd}(\mathrm{Ph})(\mathrm{O}-\mathrm{p}$-tol) (5) and reductive elimination to afford diaryl ether $3 \mathrm{e}$.

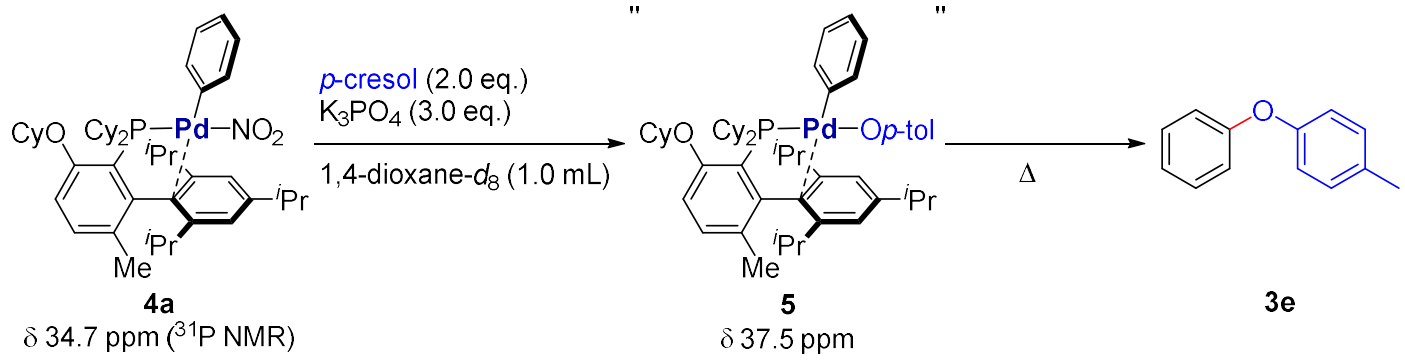

A $4 \mathrm{~mL}$ vial (A) was charged with $(\mathbf{L 9}) \mathrm{Pd}(\mathrm{Ph})\left(\mathrm{NO}_{2}\right)(4 \mathbf{a})(25 \mathrm{mg}, 18 \mu \mathrm{mol}), 1,4$-dioxane- $d_{8}(0.50$ $\mathrm{mL}$ ) and a stirring bar. A separate $4 \mathrm{~mL}$ vial (B) was charged with $p$-cresol (2b) $(3.9 \mathrm{mg}, 36 \mu \mathrm{mol})$, $\mathrm{K}_{3} \mathrm{PO}_{4}(12 \mathrm{mg}, 54 \mu \mathrm{mol}), 1,4-$ dioxane- $d_{8}(0.50 \mathrm{~mL})$ and a stirring bar. After the reaction mixture in vial (A) was stirred for 30 min at ambient temperature, the contents of vial (B) was transferred to vial (A) using a pipet. The resulting mixture was stirred for $24 \mathrm{~h}$ at ambient temperature, then filtered with 
glass fiber filter and eluted with 1,4-dioxane into a J. Young NMR tube. ${ }^{31} \mathrm{P}$ NMR (162 MHz, 1,4dioxane- $d_{8}$ ): $\delta 37.5$ (Fig. S2). Although we failed to fully characterize the obtained complex probably because of residual $\mathbf{2 b}$, we assume this would be a transmetalation product $(\mathbf{L 9}) \mathrm{Pd}(\mathrm{Ph})(\mathrm{O}-p$-tol) (5) based on ${ }^{1} \mathrm{H}$ NMR spectrum compared with an analogous complex found in the literature. ${ }^{2}$

The reaction mixture in the NMR tube was then heated at $60{ }^{\circ} \mathrm{C}$ for $1 \mathrm{~h}$, at $80{ }^{\circ} \mathrm{C}$ for $1 \mathrm{~h}$, at $100{ }^{\circ} \mathrm{C}$ for $1 \mathrm{~h}$. The reaction was monitored by ${ }^{1} \mathrm{H}$ NMR to observe the formation of $\mathbf{3 e}$ upon heating at higher temperature than $80{ }^{\circ} \mathrm{C}$ (Fig. S3). To determine the yield of 3e, another $4 \mathrm{~mL}$ vial was charged with (L9) $\mathrm{Pd}(\mathrm{Ph})\left(\mathrm{NO}_{2}\right)$ (4a) (25 mg, $\left.18 \mu \mathrm{mol}\right), p$-cresol (2b) (3.9 mg, $\left.36 \mu \mathrm{mol}\right), \mathrm{K}_{3} \mathrm{PO}_{4}(12 \mathrm{mg}, 54 \mu \mathrm{mol})$, and 1,4-dioxane $(1.0 \mathrm{~mL})$ and a stirring bar, and the resulting mixture was stirred for $24 \mathrm{~h}$ at $150{ }^{\circ} \mathrm{C}$. The yield of $3 \mathrm{e}$ based on $4 \mathrm{a}$ was calculated to be $65 \%$ by GC analysis of reaction mixture using a calibration curve (Fig. S5).

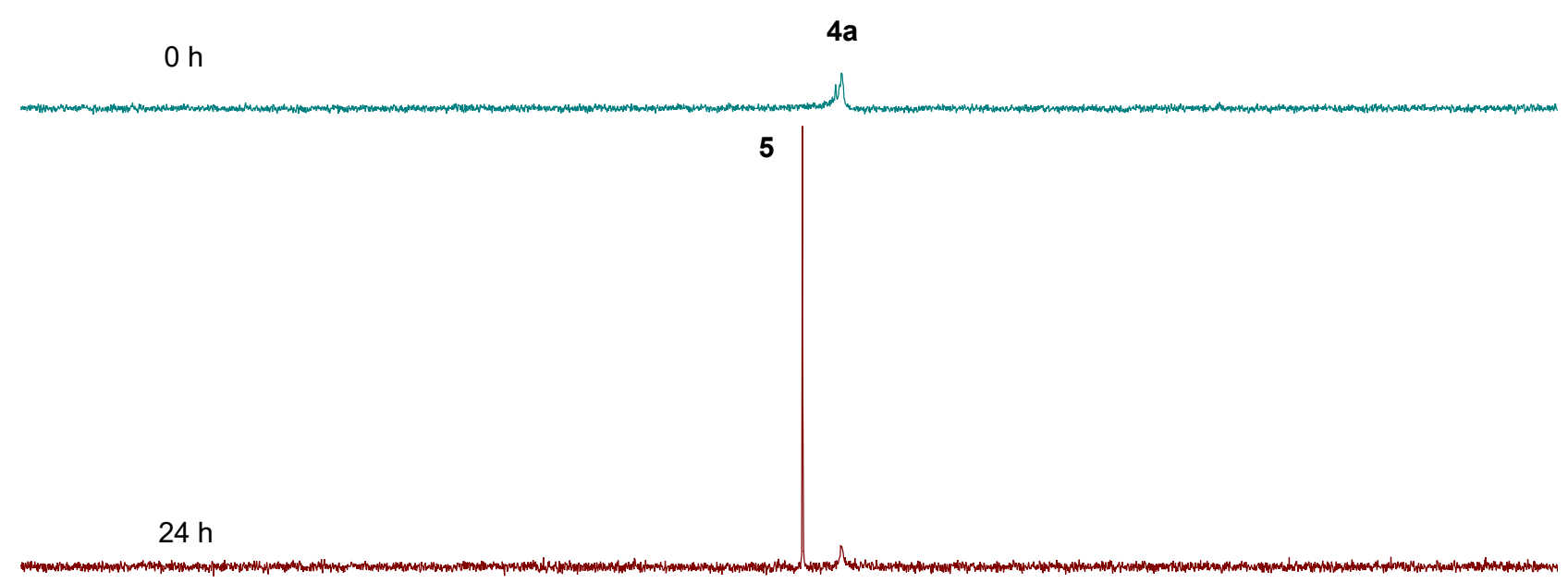

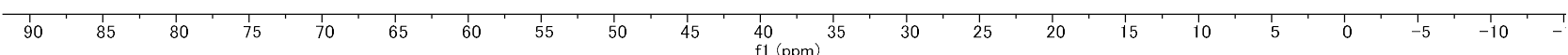

Figure S2. ${ }^{31} \mathrm{P}$ NMR spectra before and after the reaction of $\mathbf{4 a}$ and $\mathbf{2} \mathbf{b}$ at ambient temperature. 


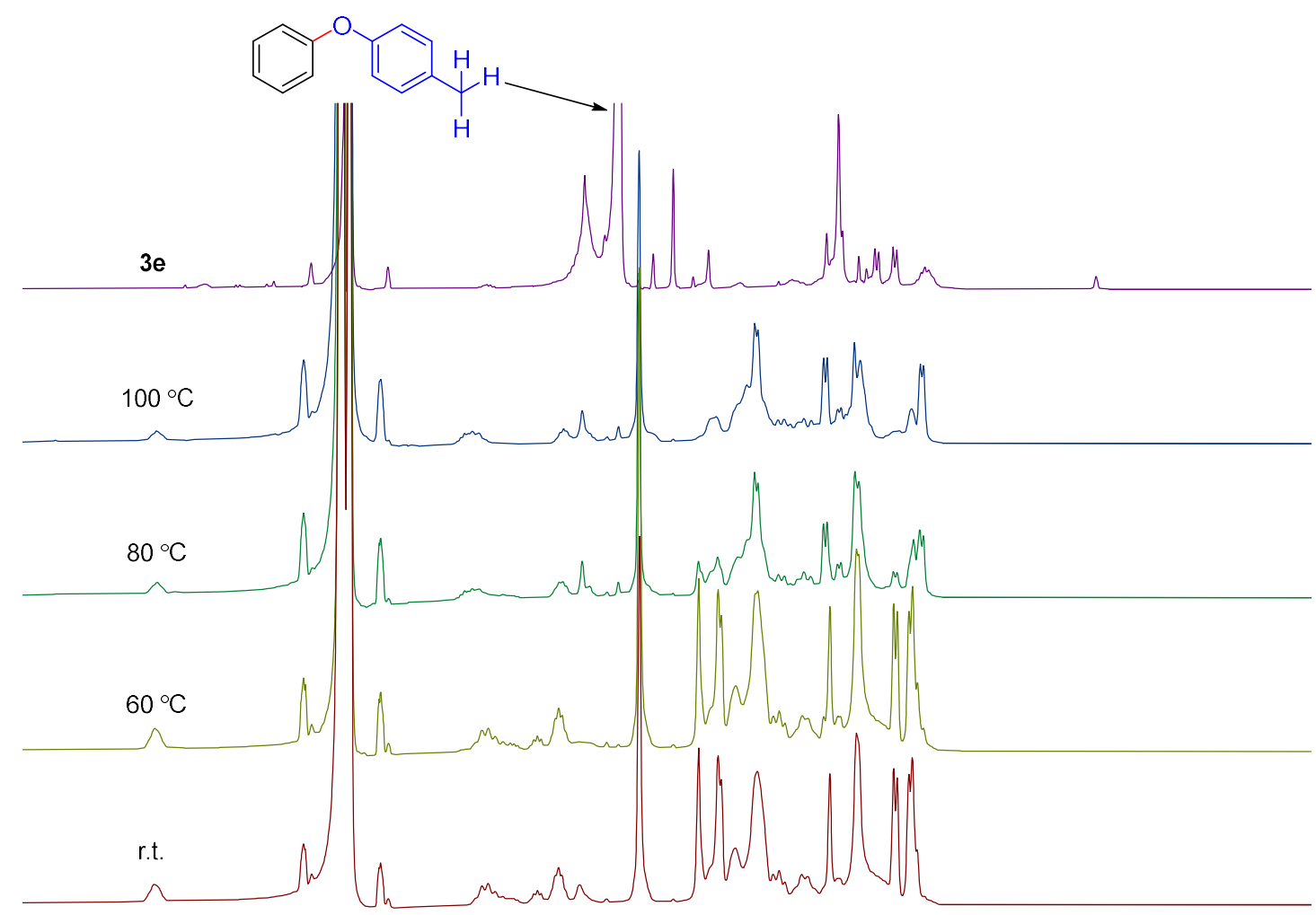

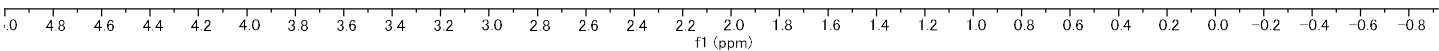

Figure S3. ${ }^{1} \mathrm{H}$ NMR monitoring upon heating of " 5 ".

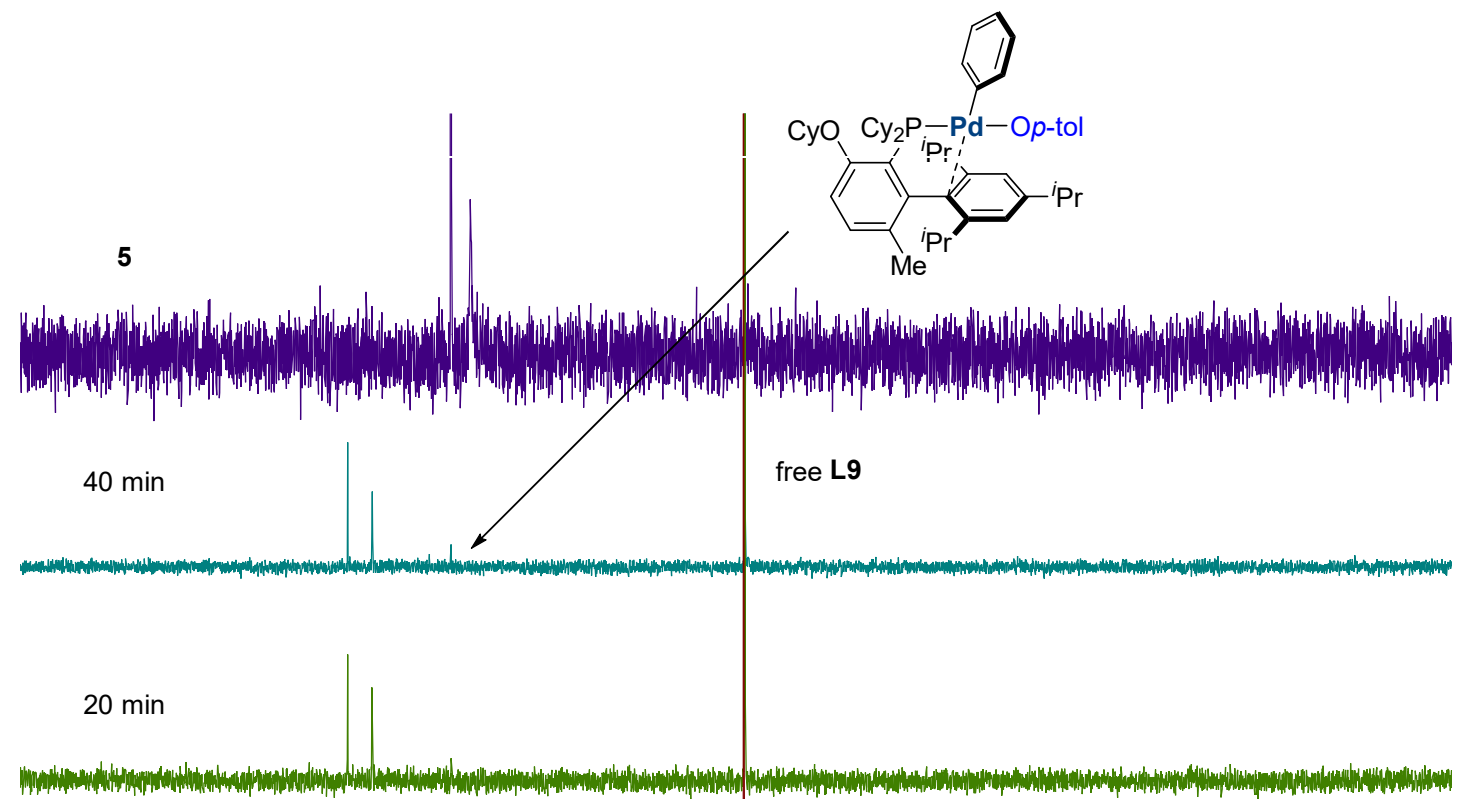

$\mathrm{Oh}$
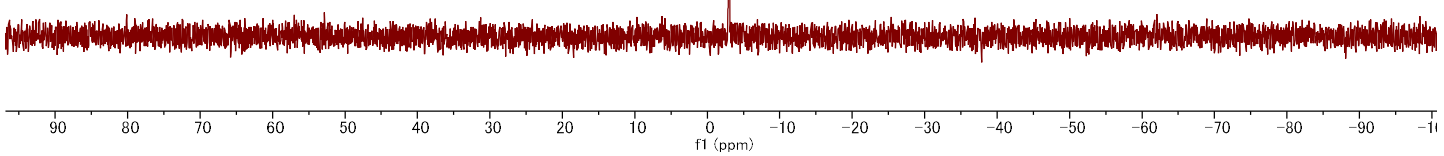

Figure S4. ${ }^{31} \mathrm{P}$ NMR monitoring during the reaction of $\mathbf{1 d}$ and $\mathbf{2 b}$ under catalytic standard conditions. The identical signal to estimated $\mathbf{5}$ appeared around $40 \mathrm{~min}$, indicating this species can be the resting state in the catalytic cycle. 
Table S1. Data for the GC calibration curve obtained using an authentic sample of 1-methyl-4phenoxybenzene (3e)

\begin{tabular}{|c|c|c|c|c|c|}
\hline \multicolumn{2}{|c|}{ mass (mg) } & \multirow{2}{*}{$\frac{\text { mass of } \mathbf{3 e}}{\text { mass of } \mathrm{C}_{13} \mathrm{H}_{28}}$} & \multicolumn{2}{|c|}{ GC area } & \multirow{2}{*}{$\begin{array}{c}\mathrm{GC} \text { area of } \mathbf{3 e} \\
\mathrm{GC} \text { area of } \mathrm{C}_{13} \mathrm{H}_{28}\end{array}$} \\
\hline $3 \mathbf{e}$ & $\mathrm{C}_{13} \mathrm{H}_{28}$ & & $3 e$ & $\mathrm{C}_{13} \mathrm{H}_{28}$ & \\
\hline 5.8 & 7.5 & 0.773333 & 6199458 & 8844709 & 0.700923 \\
\hline 9.9 & 7.4 & 1.337838 & 10873101 & 9583978 & 1.134508 \\
\hline 12.7 & 7.5 & 1.693333 & 13265164 & 9307997 & 1.425136 \\
\hline 15.2 & 7.6 & 2.000000 & 16192853 & 9491905 & 1.705964 \\
\hline 18.8 & 7.5 & 2.506667 & 18910052 & 9709914 & 1.947499 \\
\hline 23.8 & 7.4 & 3.216216 & 23941517 & 9243468 & 2.590101 \\
\hline
\end{tabular}

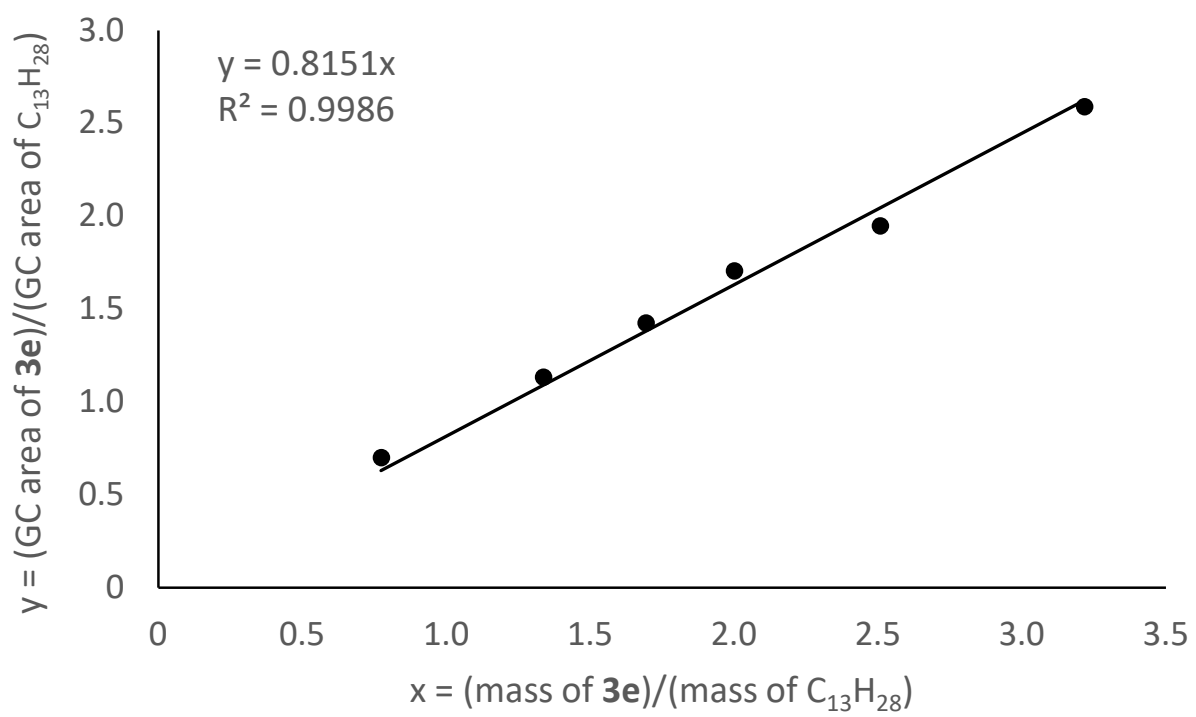

Figure S5. GC calibration curve to determine the yield of 1-methyl-4-phenoxybenzene (3e). 


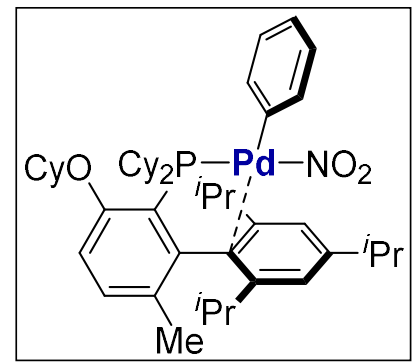

Crystals suitable for X-ray crystallography were obtained by slow diffusion of hexane into a saturated $\mathrm{CHCl}_{3}$ solution at room temperature.

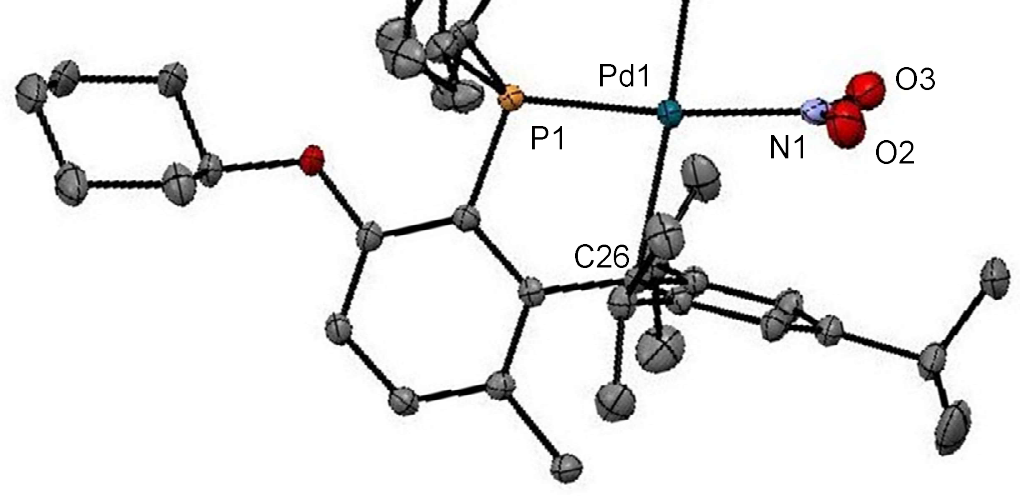

Figure S6. Crystal structure of $(\mathbf{L 9}) \mathrm{Pd}(\mathrm{Ph})\left(\mathrm{NO}_{2}\right)$ (4a) (hydrogen atoms and $\mathrm{CHCl}_{3}$ solvate molecules omitted for clarity; only selected atoms are labeled). Selected bond length ( $)$ : Pd1-N1 2.1114(18), Pd1-C42 2.017(2), Pd1-C26 2.4416(18), Pd1-P1 2.2875(6). Selected bond angles ( $\left.{ }^{\circ}\right)$ : C42-Pd1-N1 79.79(7), C42-Pd1-P1 94.09(6), P1-Pd1-C26 83.38(4), N1-Pd1-C26 102.79(6).

\section{General procedure for the synthesis of Au complexes.}
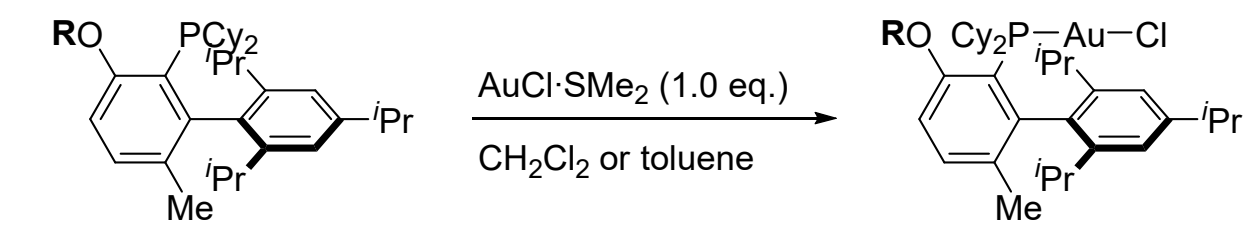

$$
\mathbf{R}=\operatorname{Me}(\mathbf{L 5}),{ }^{i} \operatorname{Pr} \text { (L7), Cy (L9) }
$$

A $4 \mathrm{~mL}$ vial was charged with $\mathbf{L 5}, \mathbf{L} 7$ or $\mathbf{L 9}(50 \mu \mathrm{mol})$ and a stirring bar. In a grove box, $\mathrm{AuCl} \cdot \mathrm{SMe}_{2}$ (26 mg, $50 \mu \mathrm{mol})$ and toluene $(1.0 \mathrm{~mL})$ were added to the vial. (In the case of $\mathbf{L 5}$, dichloromethane was used as a solvent.) The resulting mixture was stirred for $5 \mathrm{~h}$ at room temperature. The solvent was removed under reduced pressure and the residue was triturated with hexane to give the desired gold complex as a colorless solid. 


\section{Analytical data of (L5)AuCl (6b).}

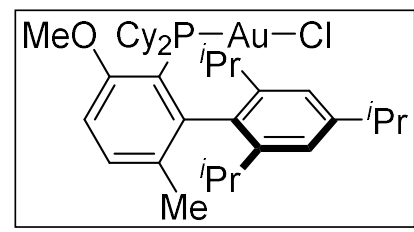

${ }^{1} \mathrm{H}$ NMR (400 MHz, $\mathrm{CDCl}_{3}$ ): $\delta 7.37$ (d, $\left.J=8.5 \mathrm{~Hz}, 1 \mathrm{H}\right), 7.06(\mathrm{~s}, 2 \mathrm{H}), 6.89$ $(\mathrm{d}, J=8.5 \mathrm{~Hz}, 1 \mathrm{H}), 3.92(\mathrm{~s}, 3 \mathrm{H}), 2.97$ (hept, $J=8.5,7.9 \mathrm{~Hz}, 1 \mathrm{H}), 2.58-2.43$ (m, 2H), 2.28 (hept, $J=6.9 \mathrm{~Hz}, 2 \mathrm{H}), 2.03-1.92(\mathrm{~m}, 2 \mathrm{H}), 1.85-1.68(\mathrm{~m}, 7 \mathrm{H})$, $1.67-1.52(\mathrm{~m}, 4 \mathrm{H}), 1.52-1.41(\mathrm{~m}, 2 \mathrm{H}), 1.36(\mathrm{~d}, J=6.8 \mathrm{~Hz}, 6 \mathrm{H}), 1.34-1.26$ $(\mathrm{m}, 8 \mathrm{H}), 1.24-1.06(\mathrm{~m}, 6 \mathrm{H}), 0.95(\mathrm{~d}, J=6.6 \mathrm{~Hz}, 6 \mathrm{H}) .{ }^{13} \mathrm{C} \mathrm{NMR}\left(101 \mathrm{MHz}, \mathrm{CDCl}_{3}\right): \delta 159.6,150.2$, $148.8(\mathrm{~d}, J=13.7 \mathrm{~Hz}), 144.8,134.8,134.6,134.5,132.8,132.7,122.3,116.2,115.8,109.4(\mathrm{~d}, J=$ $4.8 \mathrm{~Hz}), 55.4,39.4,39.1,34.5,34.5$, 34.2, 30.4, 29.6, 27.5, 27.4, 26.9, 26.7, 25.8, 25.5, 24.8, 24.5, 21.5. ${ }^{31} \mathrm{P}$ NMR (162 MHz, $\left.\mathrm{CDCl}_{3}\right): \delta$ 41.6. mp. $269.0{ }^{\circ} \mathrm{C}\left(\right.$ dec.). HRMS-ESI $(+)(\mathrm{m} / \mathrm{z}):[\mathrm{M}+\mathrm{Na}]^{+}$ calcd for $\mathrm{C}_{35} \mathrm{H}_{53} \mathrm{AuClOPNa}, 775.3080$; found, 775.3082. Anal. Calcd for $\mathrm{C}_{35} \mathrm{H}_{53} \mathrm{AuClOP}$ : C, 55.81; H, 7.09. Found: C, 55.55; H, 7.10. Crystals suitable for X-ray crystallography were obtained by slow diffusion of hexane into a saturated dichloromethane solution at room temperature.

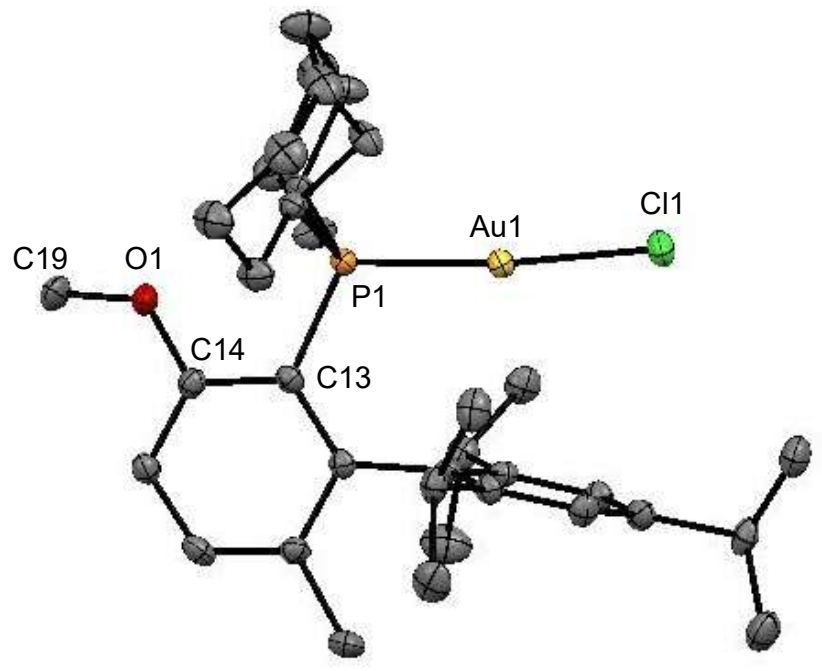

Figure S7. Crystal structure of (L5) $\mathrm{AuCl}(\mathbf{6 b})$ (hydrogen atoms omitted for clarity; only selected atoms are labeled). Selected bond length (A): Au1-P1 2.2375(8), Au1-C11 2.2950(8), P1-C13 1.841(3). Selected bond angles $\left(^{\circ}\right)$ : P1-Au1-Cl1 172.55(3), C13-P1-Au1 113.57(10). Selected dihedral angle $\left(^{\circ}\right)$ : C19-O1-C14-C13 157.3(3).

\section{Analytical data of (L7)AuCl (6c).}

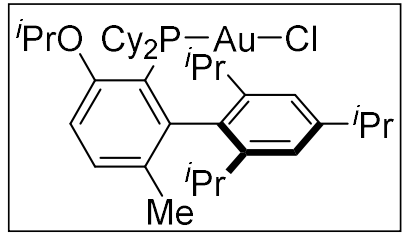

${ }^{1} \mathrm{H}$ NMR (400 MHz, $\left.\mathrm{CDCl}_{3}\right): \delta 7.32(\mathrm{~d}, J=8.2 \mathrm{~Hz}, 1 \mathrm{H}), 7.06(\mathrm{~s}, 2 \mathrm{H}), 6.82$ $(\mathrm{d}, J=8.7 \mathrm{~Hz}, 1 \mathrm{H}), 4.75$ (hept, $J=5.4 \mathrm{~Hz}, 1 \mathrm{H}), 3.04-2.90(\mathrm{~m}, 1 \mathrm{H}), 2.68-$ $2.53(\mathrm{~m}, 2 \mathrm{H}), 2.33-2.23(\mathrm{~m}, 2 \mathrm{H}), 2.04-1.91(\mathrm{~m}, 2 \mathrm{H}), 1.87-1.41(\mathrm{~m}, 20 \mathrm{H})$, $1.36(\mathrm{~d}, J=6.9 \mathrm{~Hz}, 6 \mathrm{H}), 1.33(\mathrm{~d}, J=7.0 \mathrm{~Hz}, 6 \mathrm{H}), 1.29-1.25(\mathrm{~m}, 1 \mathrm{H}), 1.24$ $1.10(\mathrm{~m}, 6 \mathrm{H}), 0.96(\mathrm{~d}, J=6.6 \mathrm{~Hz}, 6 \mathrm{H}) .{ }^{13} \mathrm{C} \mathrm{NMR}\left(101 \mathrm{MHz}, \mathrm{CDCl}_{3}\right): \delta 157.3,150.1,149.2(\mathrm{~d}, J=$ $14.1 \mathrm{~Hz}), 144.8,134.7,134.6,131.9$ (d, $J=8.1 \mathrm{~Hz}), 122.2,115.8,115.3,109.5$ (d, $J=5.1 \mathrm{~Hz}), 69.5$, 39.6, 39.2, 34.9, 34.8, 34.2, 30.3, 29.1, 27.5, 27.4, 27.0, 26.8, 25.8, 25.6, 24.8, 24.5, 21.9, 21.4. ${ }^{31} \mathrm{P}$ NMR (162 MHz, $\left.\mathrm{CDCl}_{3}\right): \delta 40.9$. mp. $274.0{ }^{\circ} \mathrm{C}\left(\right.$ dec.). HRMS-ESI $(+)(\mathrm{m} / \mathrm{z}):[\mathrm{M}+\mathrm{Na}]^{+}$calcd for 
$\mathrm{C}_{37} \mathrm{H}_{57} \mathrm{AuClOPNa}$, 803.3393; found, 803.3386. Anal. Calcd for $\mathrm{C}_{35} \mathrm{H}_{53} \mathrm{AuClOP}: \mathrm{C}, 56.88 ; \mathrm{H}, 7.35$. Found: C, 56.93; H, 7.22. Crystals suitable for X-ray crystallography were obtained by slow diffusion of hexane into a saturated benzene solution at room temperature.

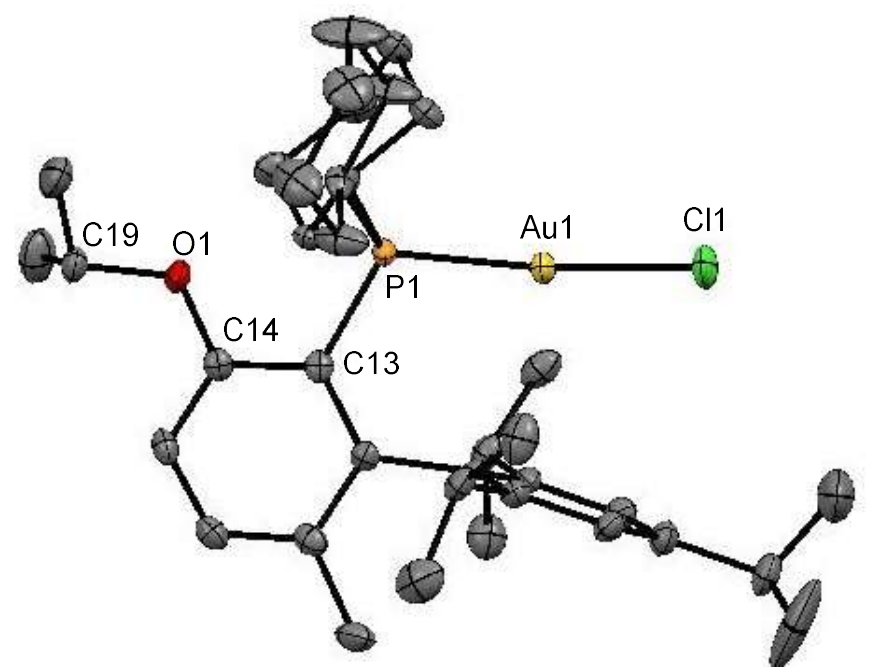

Figure S8. Crystal structure of (L7) $\mathrm{AuCl}(\mathbf{6 c})$. (hydrogen atoms omitted for clarity; only selected atoms are labeled). Selected bond length ( $)$ : Au1-P1 2.2342(13), Au1-C11 2.2851(14), P1-C13 1.839(6). Selected bond angles $\left(^{\circ}\right)$ : P1-Au1-Cl1 173.70(6), C13-P1-Au1 114.39(19). Selected dihedral angle $\left(^{\circ}\right)$ : C19-O1-C14-C13 179.3(5).

\section{Analytical data of (L9)AuCl.}

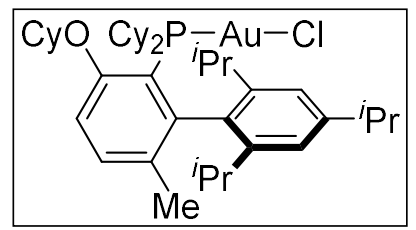

${ }^{1} \mathrm{H}$ NMR (400 MHz, CDCl3): $\delta 7.30(\mathrm{~d}, J=8.2 \mathrm{~Hz}, 1 \mathrm{H}), 7.06(\mathrm{~s}, 2 \mathrm{H}), 6.82$ $(\mathrm{d}, J=8.7 \mathrm{~Hz}, 1 \mathrm{H}), 4.45-4.33(\mathrm{~m}, 1 \mathrm{H}), 3.05-2.88(\mathrm{~m}, 1 \mathrm{H}), 2.72-2.55(\mathrm{~m}$, 2H), 2.30 (hept, $J=6.9 \mathrm{~Hz}, 2 \mathrm{H}), 2.23-2.14(\mathrm{~m}, 2 \mathrm{H}), 2.03-1.84(\mathrm{~m}, 4 \mathrm{H})$, $1.84-1.66(\mathrm{~m}, 8 \mathrm{H}), 1.66-1.41(\mathrm{~m}, 10 \mathrm{H}), 1.40-1.25(\mathrm{~m}, 15 \mathrm{H}), 1.25-1.07(\mathrm{~m}$, $6 \mathrm{H}), 0.95(\mathrm{~d}, J=6.4 \mathrm{~Hz}, 6 \mathrm{H}) .{ }^{13} \mathrm{C} \mathrm{NMR}\left(101 \mathrm{MHz}, \mathrm{CDCl}_{3}\right): \delta 150.1,149.2(\mathrm{~d}, J=14.0 \mathrm{~Hz}), 144.8$, 134.8, 134.6, 131.8 (d, $J=8.5 \mathrm{~Hz}), 122.2,115.6,115.1,109.5$ (d, $J=4.7 \mathrm{~Hz}), 75.7,39.3,38.9,34.8$, $34.7,34.2,32.0,30.3,29.1,27.5,27.4,27.0,26.8,25.7,25.6,25.5,24.8,24.5,24.2,21.5 .{ }^{31} \mathrm{P}$ NMR $\left(162 \mathrm{MHz}, \mathrm{CDCl}_{3}\right): \delta$ 41.2. mp. $276.0{ }^{\circ} \mathrm{C}$ (dec.). HRMS-ESI $(+)(\mathrm{m} / \mathrm{z}):[\mathrm{M}+\mathrm{Na}]^{+}$calcd for $\mathrm{C}_{40} \mathrm{H}_{61} \mathrm{AuClOPNa}$, 843.3706; found, 843.3704. Anal. Calcd. for $\mathrm{C}_{40} \mathrm{H}_{61} \mathrm{AuClOP}: \mathrm{C}, 58.50 ; \mathrm{H}, 7.49$. Found: C, 58.28; H, 7.39. Crystals suitable for X-ray crystallography were obtained by slow diffusion of hexane into a saturated toluene solution at room temperature. 


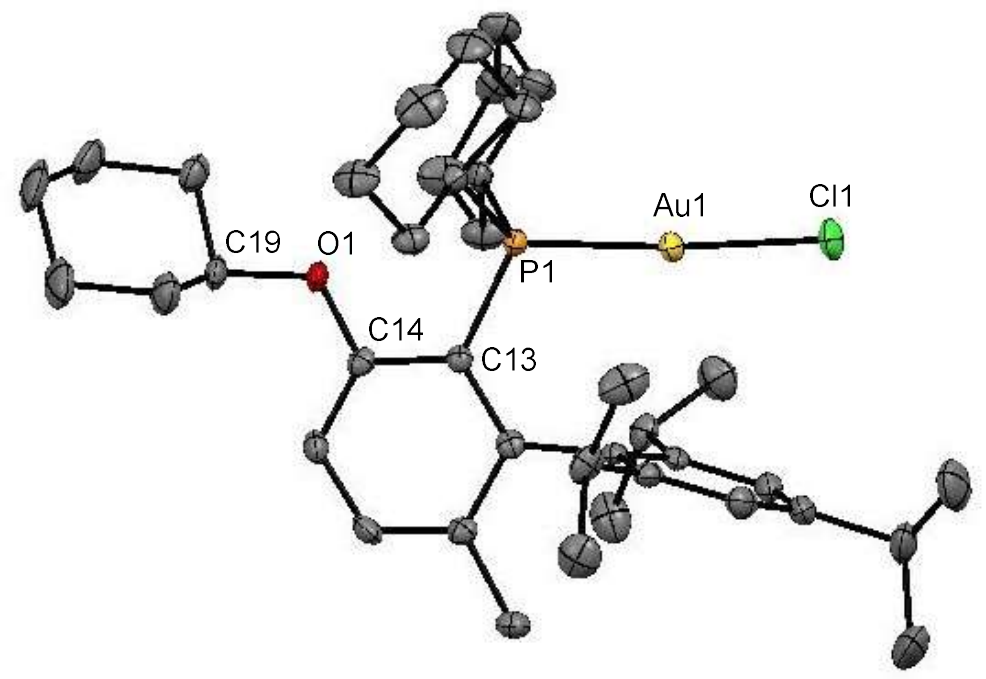

Figure S9. Crystal structure of ( $\mathbf{L 9}) \mathrm{AuCl}(\mathbf{6 d})$. (hydrogen atoms and $\mathrm{C}_{7} \mathrm{H}_{7}$ omitted for clarity; only selected atoms are labeled). Selected bond length (A): Au1-P1 2.2387(7), Au1-C11 2.2967(8), P1C13 1.833(3). Selected bond angles $\left(^{\circ}\right)$ : P1-Au1-C11 175.57(3), C13-P1-Au1 114.36(9). Selected dihedral angle $\left(^{\circ}\right)$ : C19-O1-C14-C13-175.7(2).

\section{X-ray diffraction study and X-ray crystallographic analysis.}

A crystal of $4 \mathbf{a}, \mathbf{6 b}, \mathbf{6 c}$, and $\mathbf{6 d}$ were mounted on the CryoLoop (Hampton Research Corp.) with a layer of light mineral oil and placed in a nitrogen stream at 143(1) K. The X-ray structural determination was performed on a Rigaku/Saturn724 CCD diffractometer using graphitemonochromated Mo K $\alpha$ radiation $(\lambda=0.71070 \AA)$ at $143 \mathrm{~K}$, and processed using CystalClear (Rigaku). ${ }^{12,13}$ The structures of $\mathbf{4 a}$ and $\mathbf{6 d}$ were solved by direct methods using SHELX ${ }^{14}$ and refined by full-matrix least-square refinement on $F^{2}$ with SHELXL. ${ }^{15}$ The structures of $\mathbf{6 b}$ and $\mathbf{6 c}$ were solved by direct methods using SIR92 ${ }^{16}$ and refined by full-matrix least-least-square refinement on $F^{2}$ with SHELXL. ${ }^{17}$ The non-hydrogen atoms were refined anisotropically. For all structures, the nonhydrogen atoms were refined anisotropically. All hydrogen atoms were located on the calculated positions. The function minimized was $\left[\Sigma w\left(F_{\mathrm{o}}{ }^{2}-{F_{\mathrm{c}}}^{2}\right)^{2}\right]\left(w=1 /\left[\sigma^{2}\left(F_{\mathrm{o}}{ }^{2}\right)+(a \mathrm{P})^{2}+b \mathrm{P}\right]\right)$, where $\mathrm{P}=$ $\left(\operatorname{Max}\left(F_{\mathrm{o}}^{2}, 0\right)+2{F_{\mathrm{c}}}^{2}\right) / 3$ with $\sigma^{2}\left({F_{\mathrm{o}}}^{2}\right)$ from counting statistics. The function $R 1$ and $w R 2$ were $\left(\Sigma|| F_{\mathrm{o}} \mid-\right.$ $\left.\left|F_{\mathrm{c}}\right| \mid\right) / \Sigma\left|F_{\mathrm{o}}\right|$ and $\left[\Sigma w\left(F_{\mathrm{o}}{ }^{2}-F_{\mathrm{c}}{ }^{2}\right)^{2} / \Sigma\left(w F_{\mathrm{o}}{ }^{4}\right)\right]^{1 / 2}$, respectively. CCDC 2067444, 2067443, 2060380, and 2057920 (Depositions Number) contain the supplementary crystallographic data. These data can be obtained from The Cambridge Crystallographic Data Centre. 
Table S2. Crystallographic data of 4a.

\begin{tabular}{|c|c|}
\hline Compound & $4 a$ \\
\hline empirical formula & $\mathrm{C}_{46} \mathrm{H}_{66} \mathrm{NO}_{3} \mathrm{PPd}, \mathrm{CHCl}_{3}$ \\
\hline formula weight & 937.73 \\
\hline crystal system & monoclinic \\
\hline space group & $P 2{ }_{1} / c(\# 14)$ \\
\hline$a, \AA$ & $17.041(2)$ \\
\hline$b, \AA$ & $10.8543(12)$ \\
\hline$c, \AA$ & $25.171(3)$ \\
\hline$\alpha$, deg. & 90 \\
\hline$\beta$, deg. & $98.532(2)$ \\
\hline$\gamma$, deg. & 9 \\
\hline$V, \AA^{3}$ & $4604.3(9)$ \\
\hline$Z$ & 4 \\
\hline$D_{\text {calcd. }}, \mathrm{g} / \mathrm{cm}^{-3}$ & 1.353 \\
\hline$\mu[\mathrm{Mo}-K \alpha], \mathrm{mm}^{-1}$ & 0.652 \\
\hline$T, \mathrm{~K}$ & 143 \\
\hline crystal size, $\mathrm{mm}$ & $0.120 \times 0.090 \times 0.060$ \\
\hline$\theta$ range, deg. & 3.10 to 27.50 \\
\hline reflections measured & 14327 \\
\hline unique data & 10543 \\
\hline restraints & 0 \\
\hline parameters & 523 \\
\hline$R 1(I>2.0 \sigma(I))$ & 0.0356 \\
\hline$w R 2(I>2.0 \sigma(I))$ & 0.0843 \\
\hline$R 1$ (all data) & 0.0405 \\
\hline$w R 2$ (all data) & 0.0874 \\
\hline GOF on $F^{2}$ & 1.0 \\
\hline
\end{tabular}

Table S3. Crystallographic data of $\mathbf{6 b}, \mathbf{6 c}$, and $\mathbf{6 d}$.

\begin{tabular}{|c|c|c|c|}
\hline Compound & $6 b$ & $6 c$ & 6d \\
\hline empirical formula & $\mathrm{C}_{35} \mathrm{H}_{53} \mathrm{AuClOP}$ & $\mathrm{C}_{37} \mathrm{H}_{57} \mathrm{AuClOP}$ & $2\left(\mathrm{C}_{40} \mathrm{H}_{61} \mathrm{AuClOP}\right), \mathrm{C}_{7} \mathrm{H}_{7}$ \\
\hline formula weight & 753.20 & 781.25 & 1733.67 \\
\hline crystal system & monoclinic & orthorhombic & triclinic \\
\hline space group & $P 2{ }_{1} / c(\# 14)$ & $P 22_{1} 2_{1} 2_{1}(\# 19)$ & $P \overline{1}$ \\
\hline$a, \AA$ & $9.9629(9)$ & $9.9243(14)$ & $11.5108(16)$ \\
\hline$b, \AA$ & $14.1957(12)$ & $15.578(2)$ & $12.398(2)$ \\
\hline$c, \AA$ & $23.797(3)$ & $23.092(3)$ & $16.701(3)$ \\
\hline$\alpha, \mathrm{deg}$. & 90 & 90 & $70.526(8)$ \\
\hline$\beta$, deg. & $98.246(5)$ & 90 & $70.491(6)$ \\
\hline$\gamma$, deg. & 90 & 90 & $71.5290(10)$ \\
\hline$V, \AA^{3}$ & $3330.8(6)$ & $3570.0(8)$ & $2060.7(6)$ \\
\hline$Z$ & 4 & 4 & 1 \\
\hline$D_{\text {calcd. }}, \mathrm{g} / \mathrm{cm}^{-3}$ & 1.502 & 1.453 & 1.397 \\
\hline$\mu[\mathrm{Mo}-K \alpha], \mathrm{mm}^{-1}$ & 4.585 & 4.281 & 3.704 \\
\hline$T, \mathrm{~K}$ & 143 & 293 & 143 \\
\hline crystal size, mm & $0.180 \times 0.160 \times 0.090$ & $0.120 \times 0.110 \times 0.070$ & $0.210 \times 0.130 \times 0.11$ \\
\hline$\theta$ range, deg. & 3.22 to 27.53 & 3.00 to 27.51 & 3.23 to 27.49 \\
\hline reflections measured & 10029 & 29183 & 16587 \\
\hline unique data & 7612 & 8116 & 8991 \\
\hline & & S19 & \\
\hline
\end{tabular}




$\begin{array}{llll}\text { restraints } & 0 & 0 & 9 \\ \text { parameters } & 352 & 370 & 436 \\ R 1(I>2.0 \sigma(I)) & 0.0286 & 0.0327 & 0.0279 \\ w R 2(I>2.0 \sigma(I)) & 0.0587 & 0.0484 & 0.0545 \\ R 1 \text { (all data) } & 0.0345 & 0.0361 & 0.0327 \\ w R 2 \text { (all data) } & 0.0609 & 0.0496 & 0.0560 \\ \text { GOF on } F^{2} & 1.046 & 0.977 & 0.990\end{array}$




\section{Computational details}

All geometry optimizations were performed by the density functional theory (DFT) with B3PW91 ${ }^{18}$. D3 functional ${ }^{19}$ in the gas phase because this functional reproduced the geometry of (L9)AuCl (6d) as shown below. The LANL2DZ basis sets were used for Au atom with Los Alamos effective core potential (ECP). ${ }^{20}$ The 6-31G(d) basis sets were used for all other atoms. ${ }^{21}$ All DFT calculations were performed using Gaussian $16 .^{22}$ The Gibbs energy change was evaluated at $298.15 \mathrm{~K}$ and $1 \mathrm{~atm}$.

\section{Selection of functionals.}

Based on the crystal structure of $(\mathbf{L 9}) \mathrm{AuCl}(\mathbf{6 d})$, several functionals were tested for the geometry optimization comparing with the experimental results. The $\mathrm{Au}-\mathrm{Cl}, \mathrm{Au}-\mathrm{P}, \mathrm{Au}-\mathrm{C} 1, \mathrm{P}-\mathrm{C} 20, \mathrm{P}-\mathrm{C} 31, \mathrm{P}-$ $\mathrm{C} 12, \mathrm{C} 11-\mathrm{O}$, and $\mathrm{C} 18-\mathrm{O}$ distance have been taken in this work.

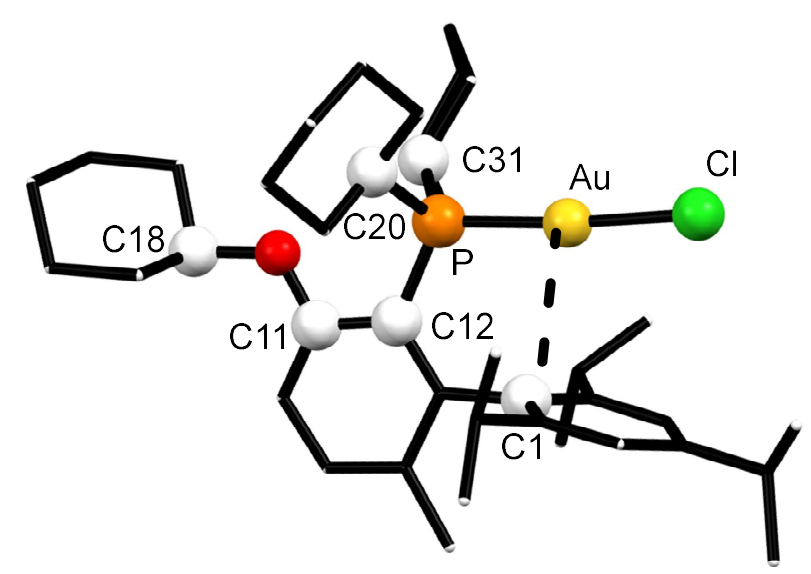

Figure S10. Crystal structure of $\mathbf{6 d}$ and definition of atom labels.

Table S4. Tests of functionals for geometry optimization.

\begin{tabular}{lcccccc} 
(in $\AA$ ) & crystal & $\omega B$ 97XD & $\omega$ B97XD-D3 & M06L & B3LYP-D3 & B3PW91-D3 \\
\hline $\mathrm{R}_{\mathrm{Au}-\mathrm{Cl}}$ & 2.297 & 2.325 & 2.350 & 2.375 & 2.369 & 2.348 \\
$\mathrm{R}_{\mathrm{Au}-\mathrm{P}}$ & 2.239 & 2.300 & 2.311 & 2.301 & 2.310 & 2.285 \\
$\mathrm{R}_{\mathrm{P}-\mathrm{C} 20}$ & 1.856 & 1.871 & 1.870 & 1.870 & 1.885 & 1.873 \\
$\mathrm{R}_{\mathrm{P}-\mathrm{C} 31}$ & 1.856 & 1.863 & 1.862 & 1.863 & 1.876 & 1.865 \\
$\mathrm{R}_{\mathrm{P}-\mathrm{C} 12}$ & 1.834 & 1.844 & 1.842 & 1.836 & 1.849 & 1.839 \\
$\mathrm{R}_{\mathrm{C} 11-\mathrm{O}}$ & 1.367 & 1.362 & 1.362 & 1.361 & 1.369 & 1.363 \\
$\mathrm{R}_{\mathrm{C} 18-\mathrm{O}}$ & 1.448 & 1.434 & 1.435 & 1.439 & 1.447 & 1.439 \\
$\mathrm{R}_{\mathrm{Au}-\mathrm{C} 1}$ & 3.040 & 3.138 & 3.145 & 3.089 & 3.152 & 3.082 \\
$\mathrm{RMSE}$ & & 0.043 & 0.049 & 0.040 & 0.551 & 0.030
\end{tabular}


7. NMR spectra
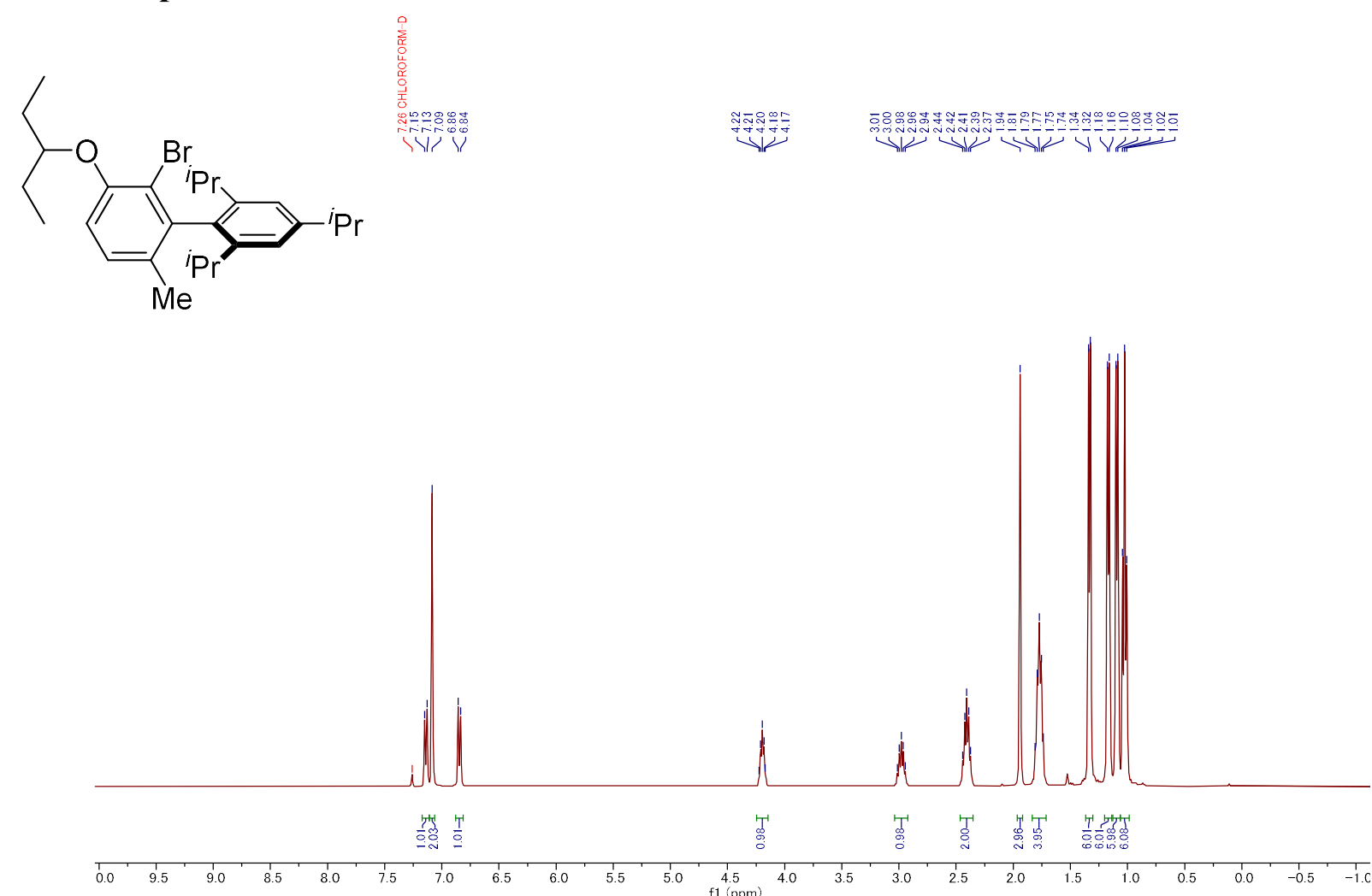

Figure S11. ' H NMR (400 MHz, $\mathrm{CDCl}_{3}$ ) of 2-bromo-2',4',6'-triisopropyl-6-methyl-3-(3-pentyloxy)1,1 '-biphenyl.
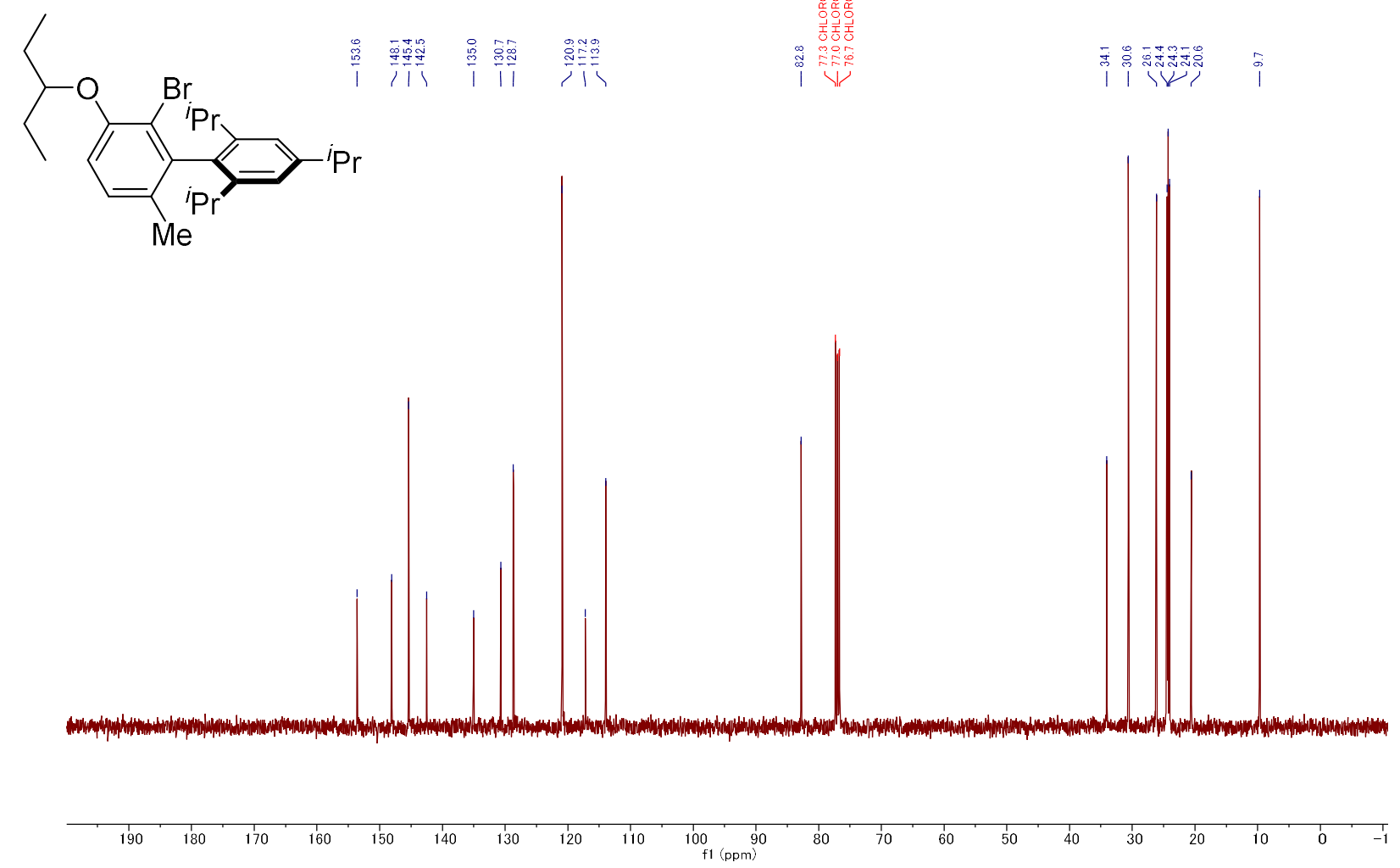

Figure S12. ${ }^{13} \mathrm{C}$ NMR (101 $\left.\mathrm{MHz}, \mathrm{CDCl}_{3}\right)$ of 2-bromo-2',4',6'-triisopropyl-6-methyl-3-(3pentyloxy)-1,1'-biphenyl. 

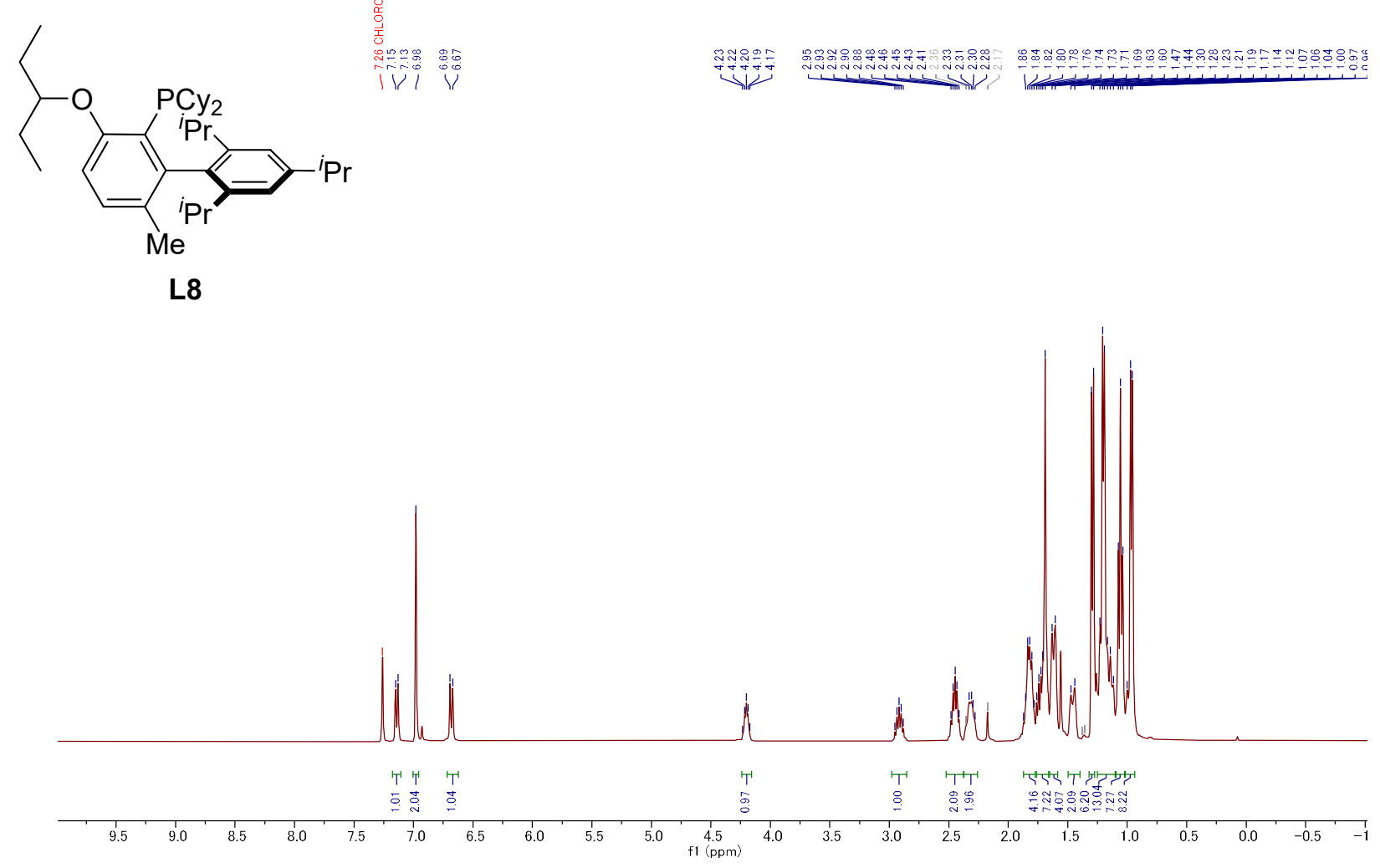

Figure S13. ${ }^{1} \mathrm{H}$ NMR (400 $\left.\mathrm{MHz}, \mathrm{CDCl}_{3}\right)$ of di-cyclohexyl-(2',4',6'-triisopropyl-6-methyl-3-(3pentyloxy)-[1,1'-biphenyl]-2-yl)phosphine (L8).
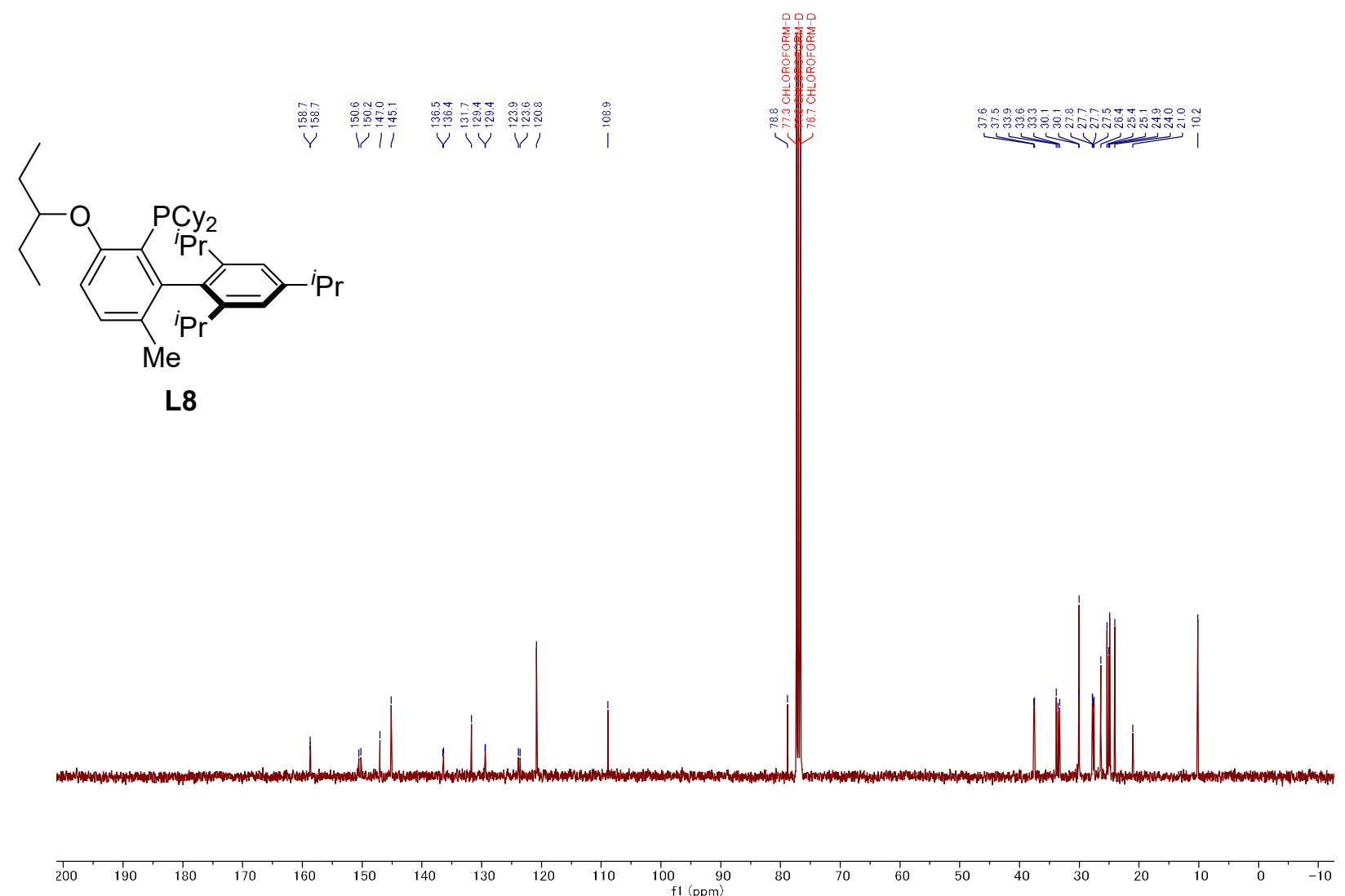

Figure S14. ${ }^{13} \mathrm{C}$ NMR (101 MHz, $\left.\mathrm{CDCl}_{3}\right)$ of di-cyclohexyl-(2',4',6'-triisopropyl-6-methyl-3-(3pentyloxy)-[1,1'-biphenyl]-2-yl)phosphine (L8). 


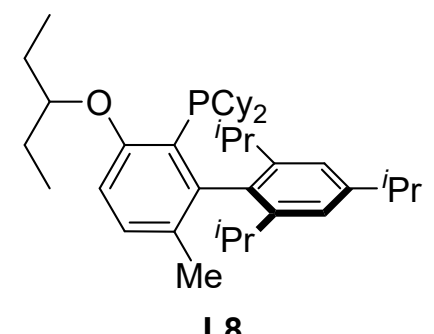

L8
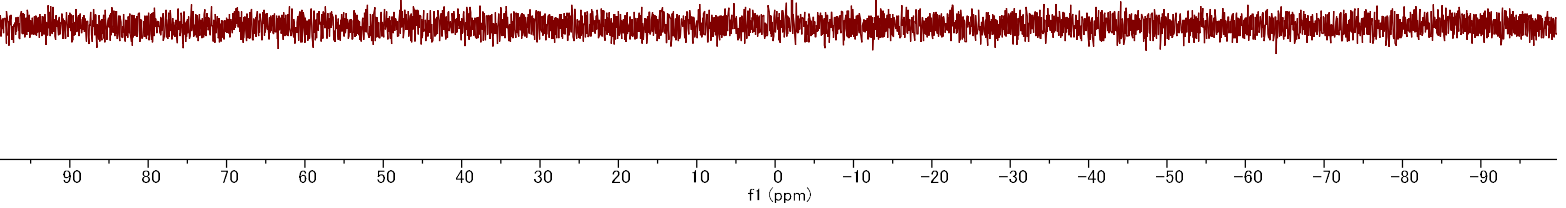

Figure S15. ${ }^{31} \mathrm{P}$ NMR (162 MHz, $\left.\mathrm{CDCl}_{3}\right)$ of di-cyclohexyl-(2',4',6'-triisopropyl-6-methyl-3-(3pentyloxy)-[1,1'-biphenyl]-2-yl)phosphine (L8)
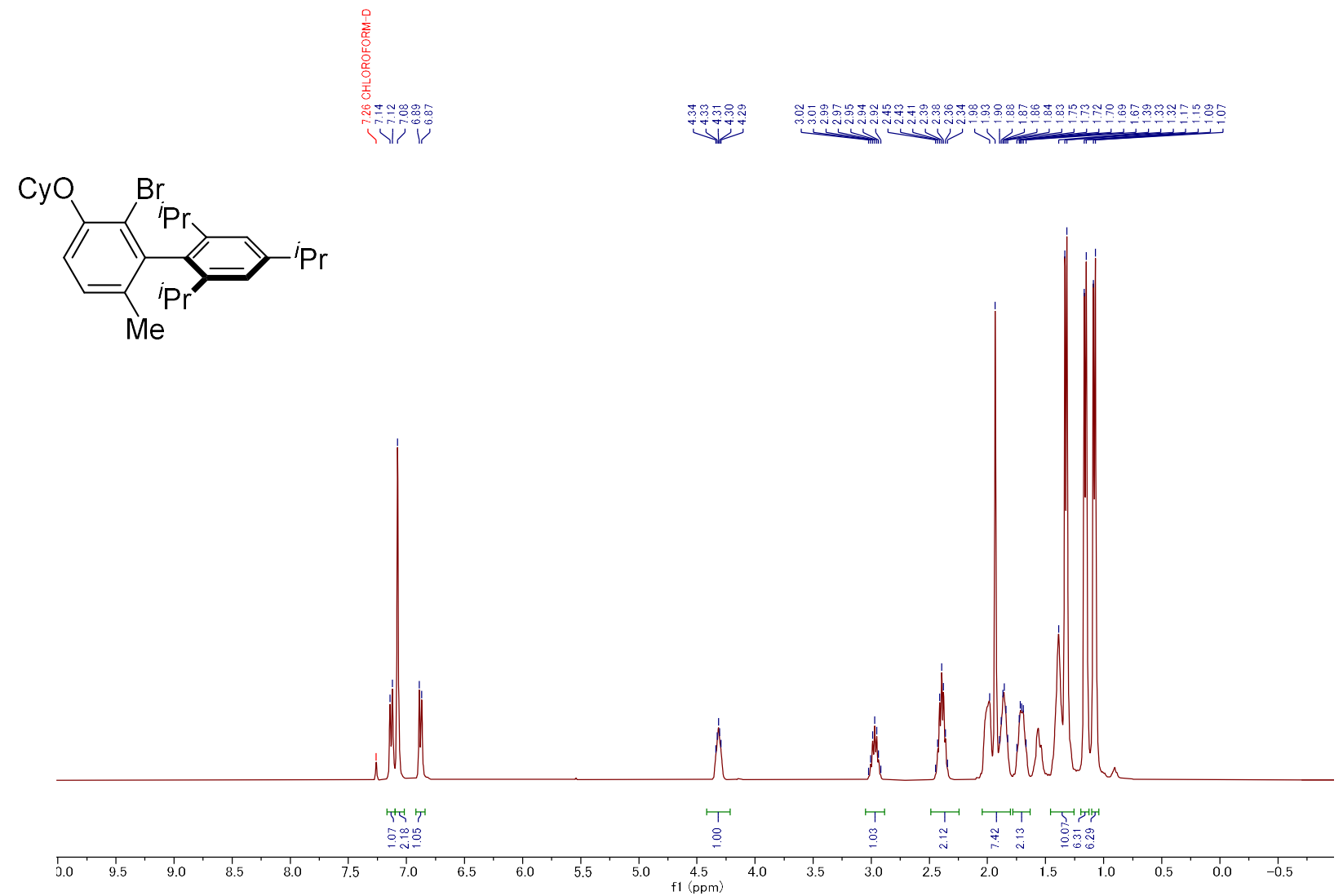

Figure S16. ${ }^{1} \mathrm{H} \mathrm{NMR}\left(400 \mathrm{MHz}, \mathrm{CDCl}_{3}\right)$ of 2-bromo-3-cyclohexyloxy-2',4',6'-triisopropyl-6methyl-1,1'-biphenyl. 


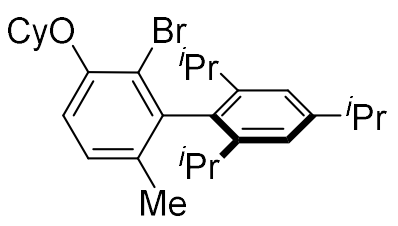
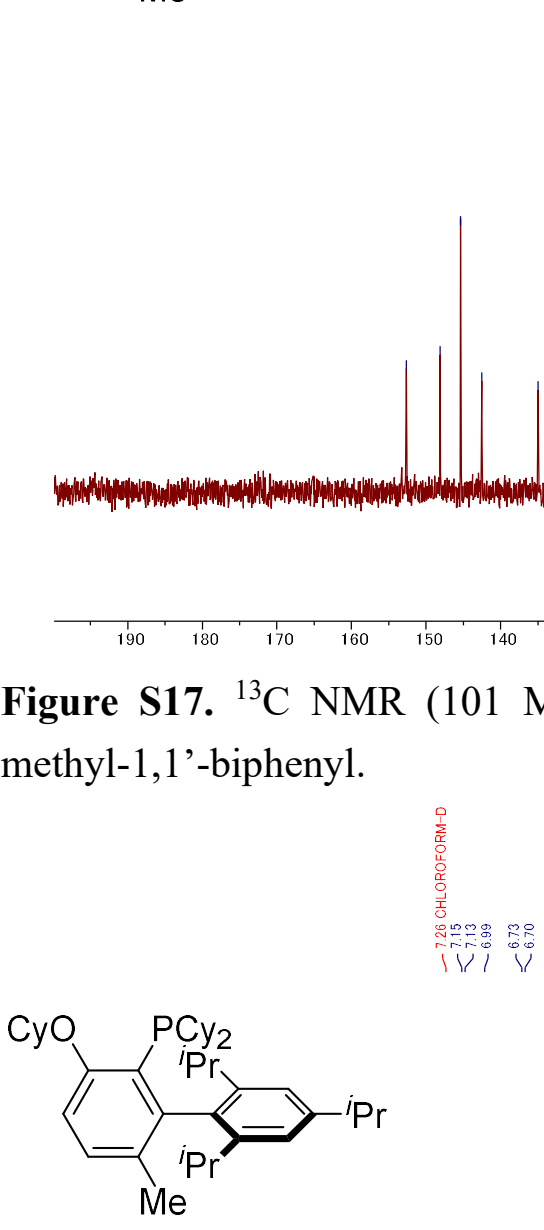

L9
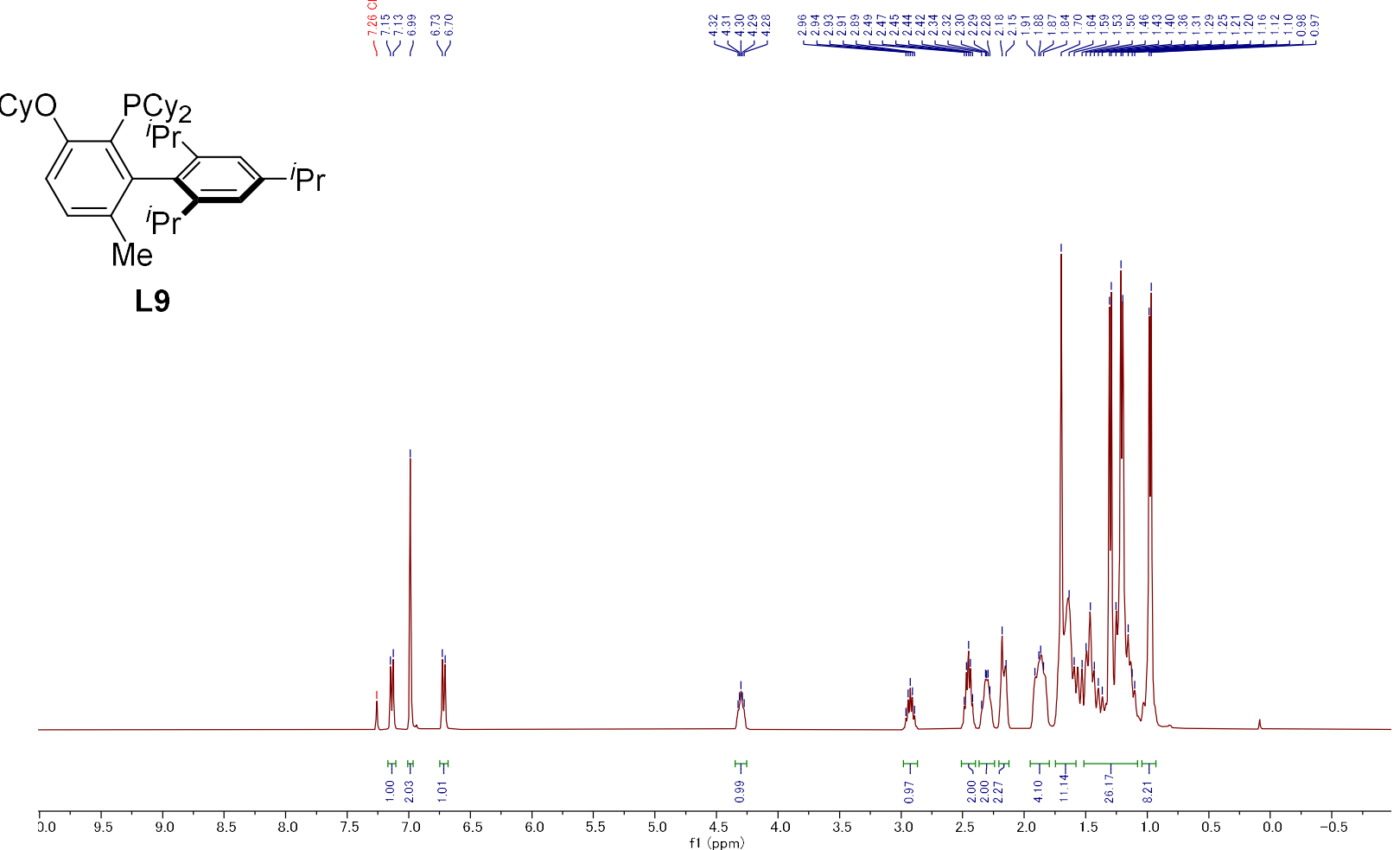

Figure S18. ' $\mathrm{H}$ NMR (400 MHz, $\mathrm{CDCl}_{3}$ ) of di-cyclohexyl-(3-(cyclohexyloxy)-2',4',6'-triisopropyl6-methyl-[1,1'-biphenyl]-2-yl)phosphine (L9). 

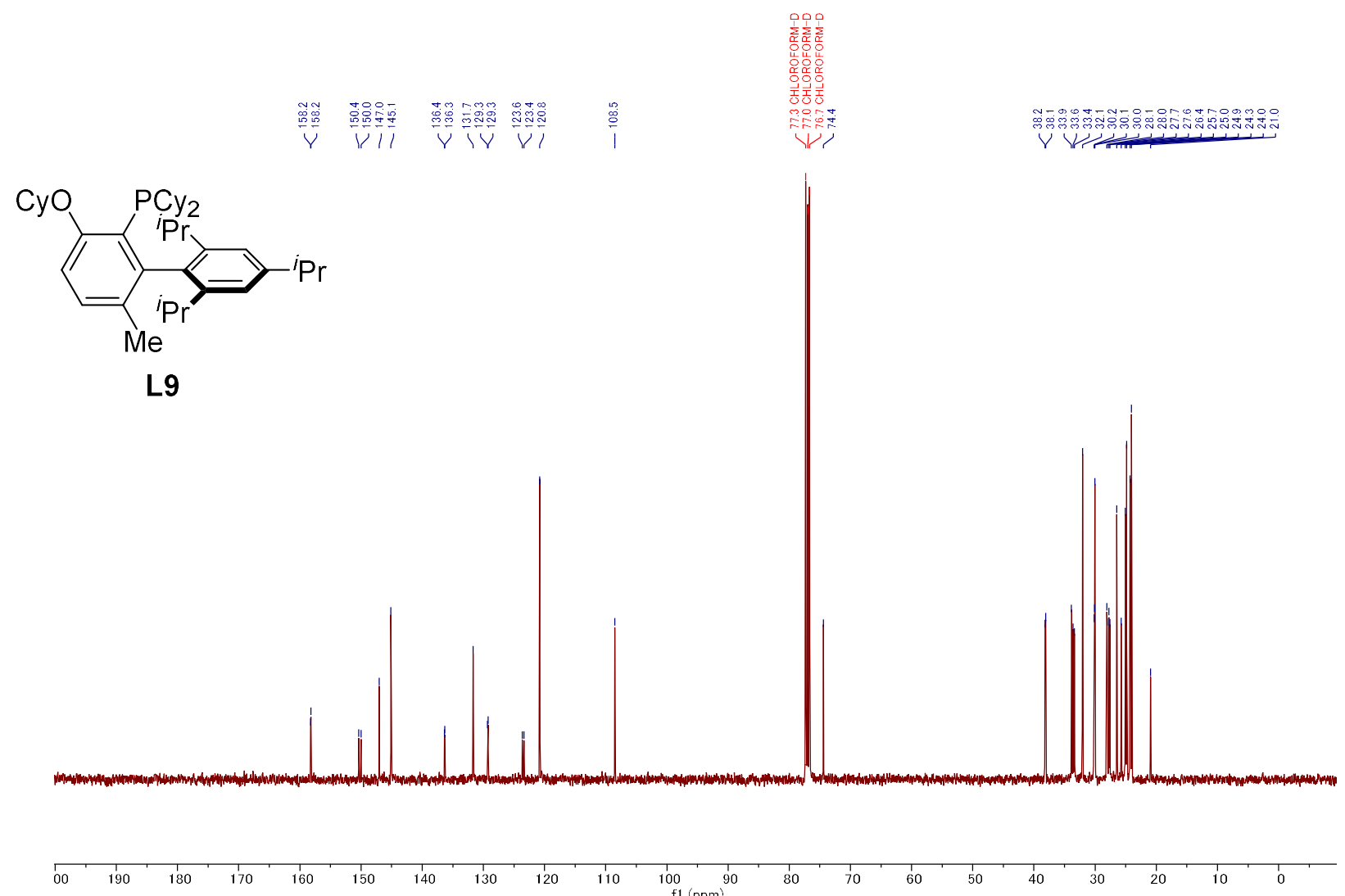

Figure S19. ${ }^{13} \mathrm{C}$ NMR (101 MHz, $\mathrm{CDCl}_{3}$ ) of di-cyclohexyl-(3-(cyclohexyloxy)-2',4',6'-triisopropyl6-methyl-[1,1'-biphenyl]-2-yl)phosphine (L9).

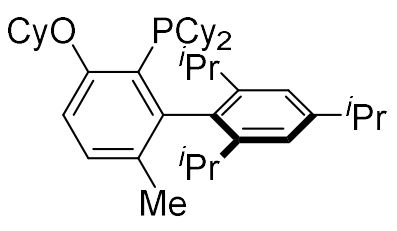

L9

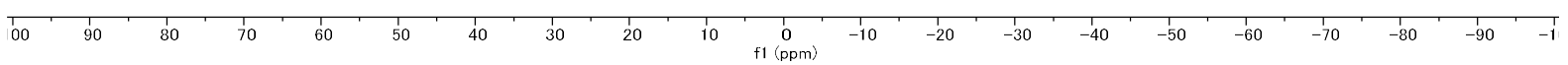

Figure S20. ${ }^{31} \mathrm{P}$ NMR (162 MHz, CDCl 3 ) of di-cyclohexyl-(3-(cyclohexyloxy)-2',4',6'-triisopropyl6-methyl-[1,1'-biphenyl]-2-yl)phosphine (L9). 


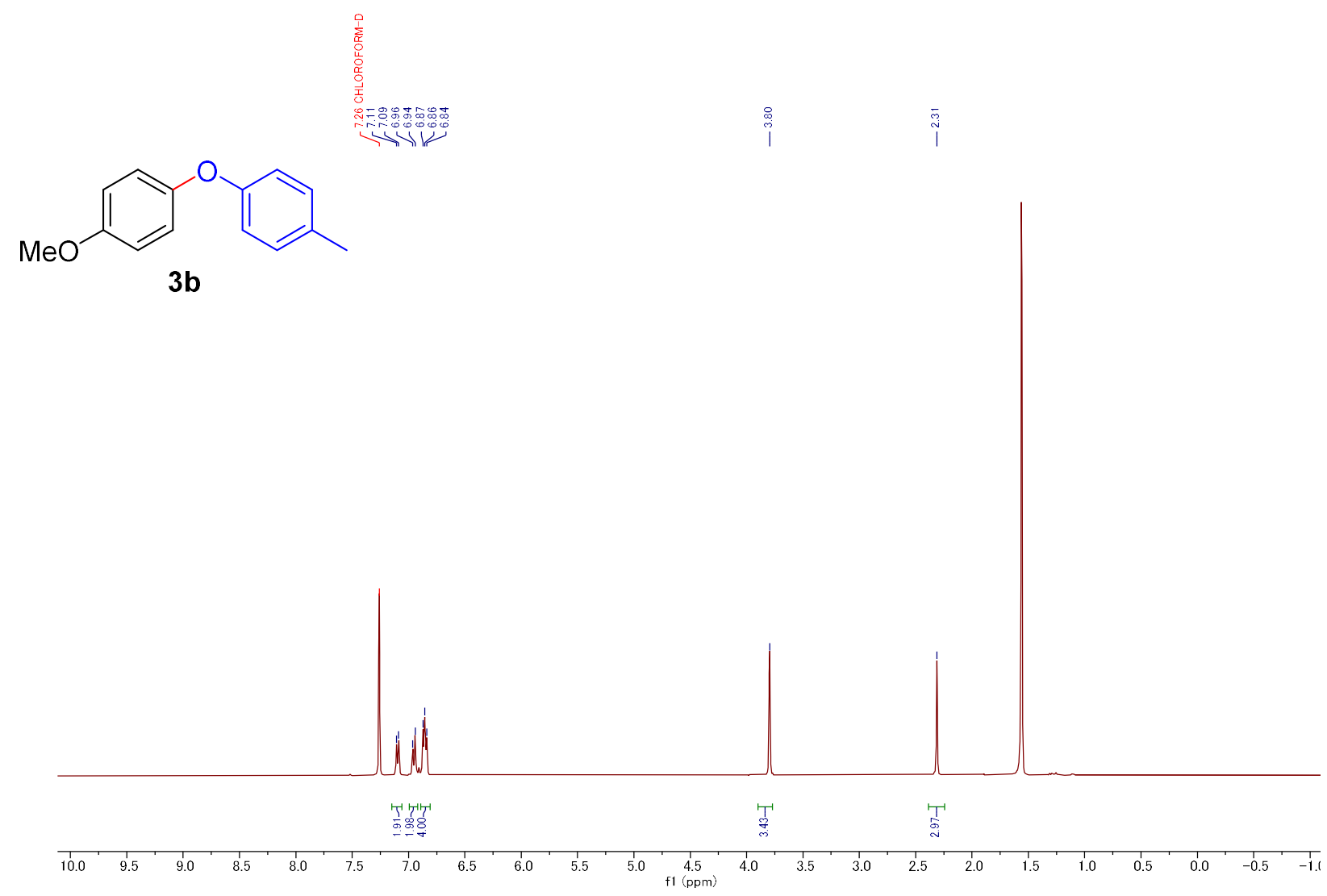

Figure S21. ${ }^{1} \mathrm{H}$ NMR (400 MHz, $\left.\mathrm{CDCl}_{3}\right)$ of 1-methoxy-4-(p-tolyloxy)benzene (3b).
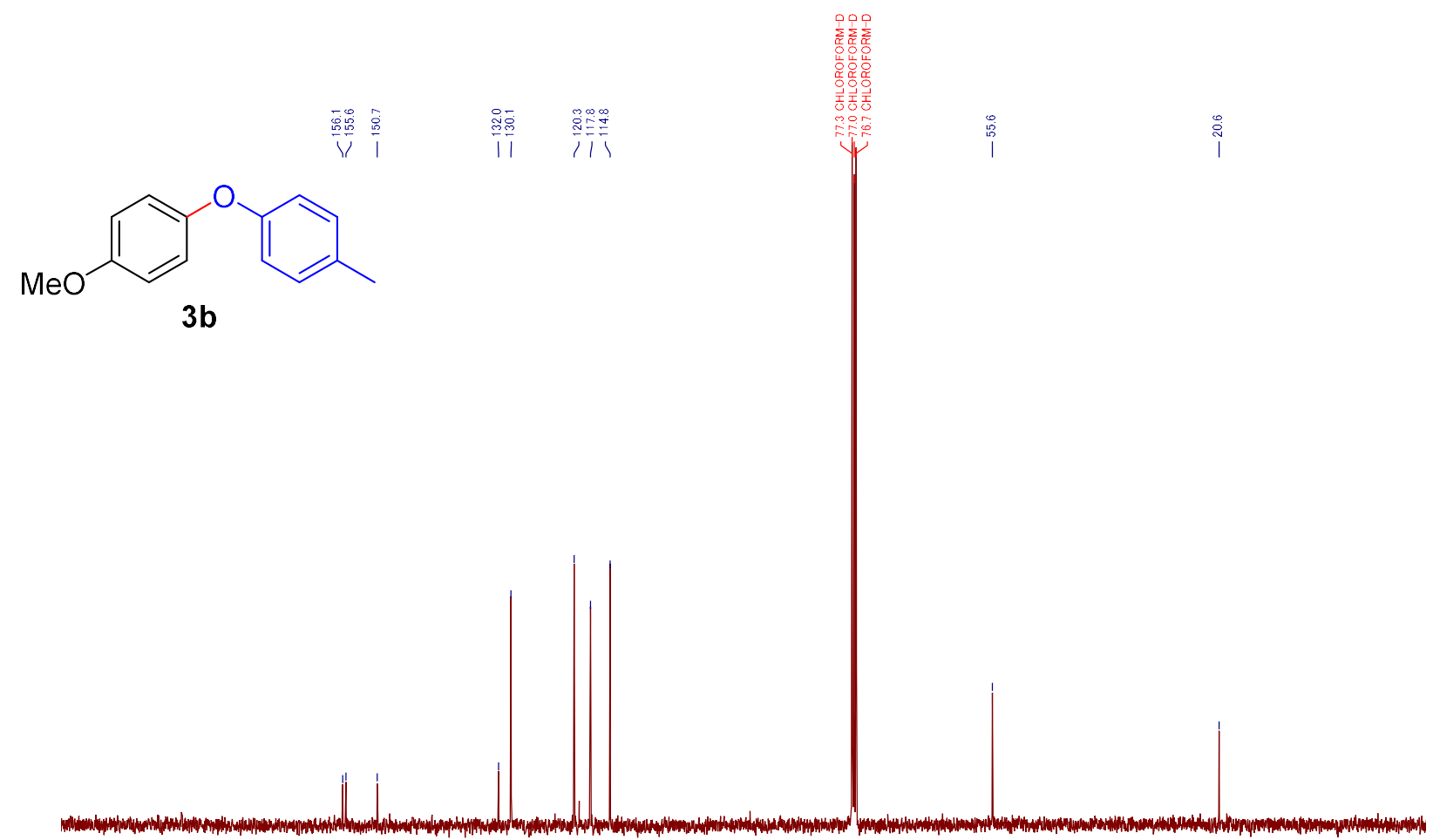

Figure S22. ${ }^{13} \mathrm{C}$ NMR (101 MHz, $\left.\mathrm{CDCl}_{3}\right)$ of 1-methoxy-4-( $p$-tolyloxy)benzene (3b). 

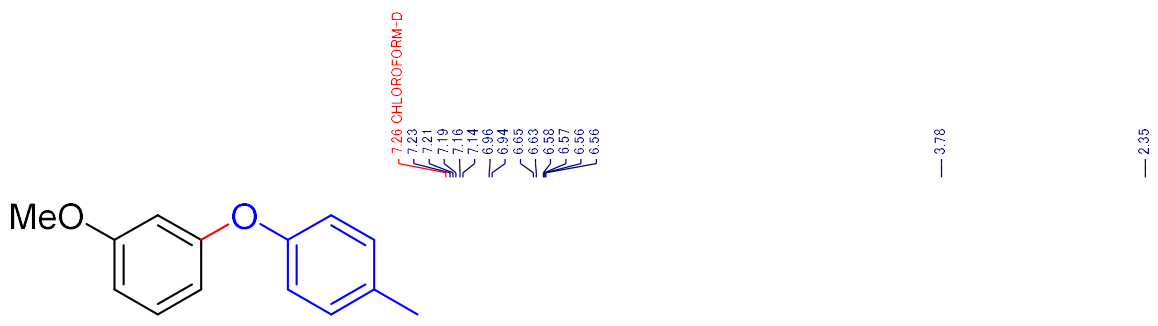

$3 c$

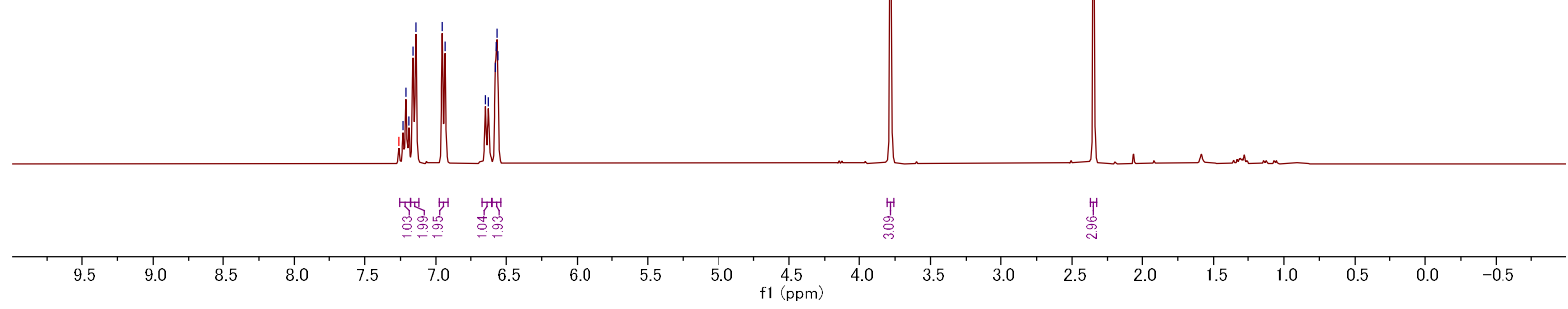

Figure S23. ${ }^{1} \mathrm{H}$ NMR (400 MHz, $\left.\mathrm{CDCl}_{3}\right)$ of 1-methoxy-3-( $p$-tolyloxy)benzene (3c).
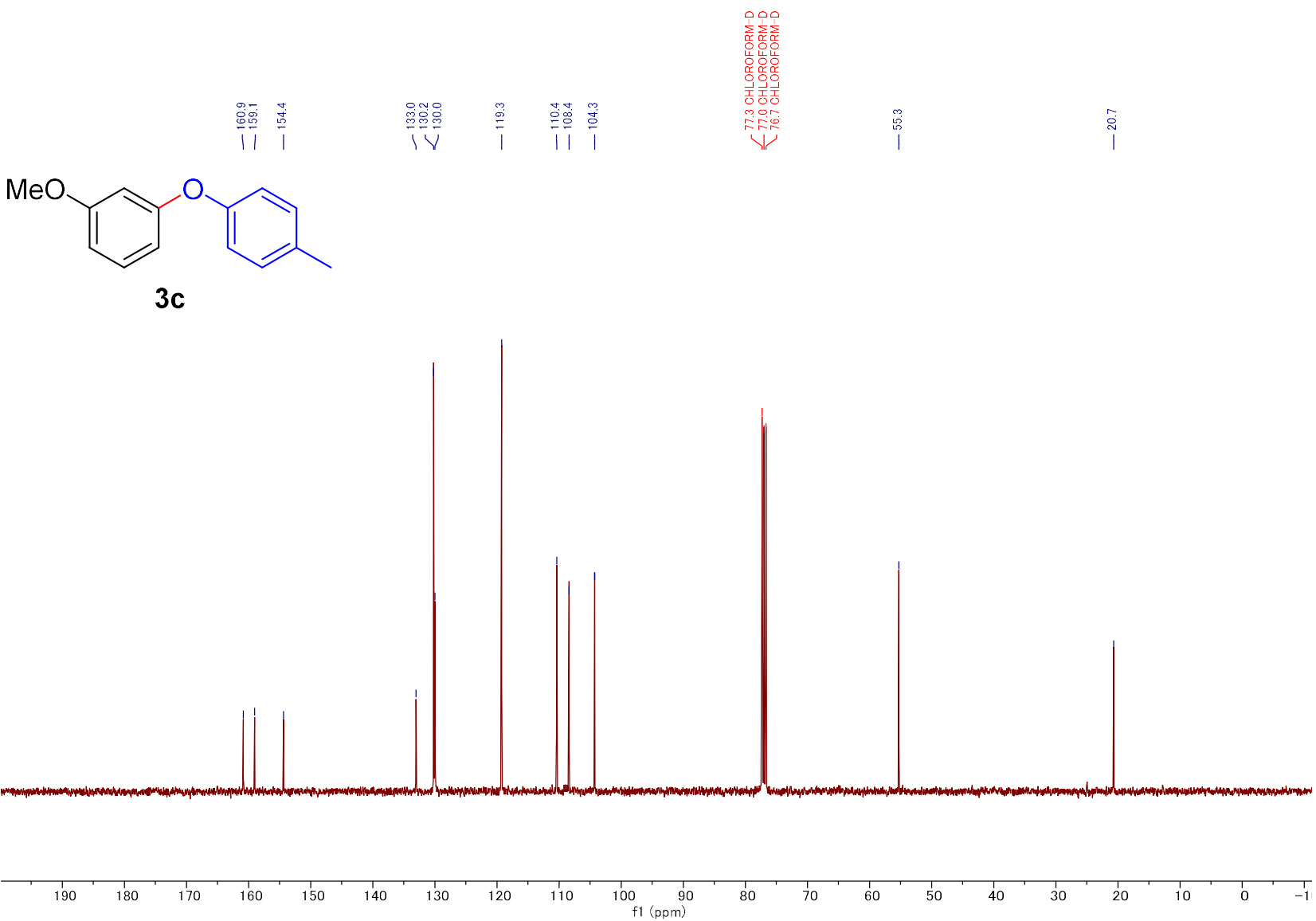

Figure S24. ${ }^{13} \mathrm{C}$ NMR (101 MHz, $\left.\mathrm{CDCl}_{3}\right)$ of 1-methoxy-3-(p-tolyloxy)benzene (3c)

S28 


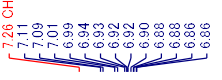

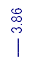

$\stackrel{\overbrace{}}{\tilde{\pi}}$

OMe

$3 d$

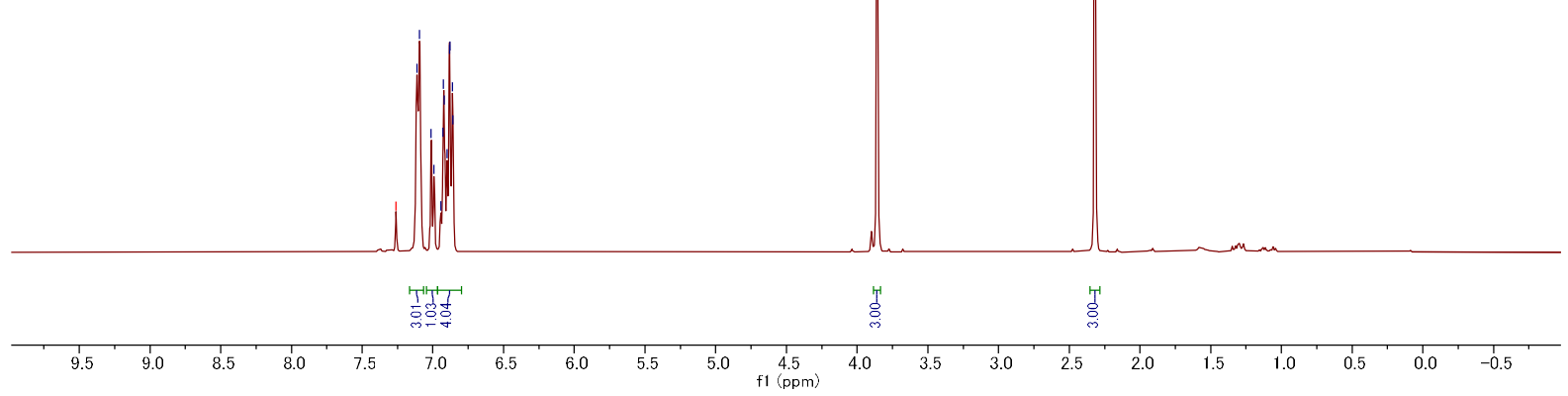

Figure S25. ${ }^{1} \mathrm{H}$ NMR (400 MHz, $\mathrm{CDCl}_{3}$ ) of 1-methoxy-2-( $p$-tolyloxy)benzene (3d).

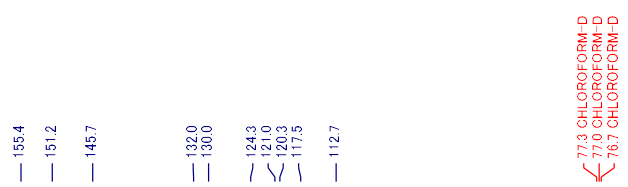

OMe

$3 d$
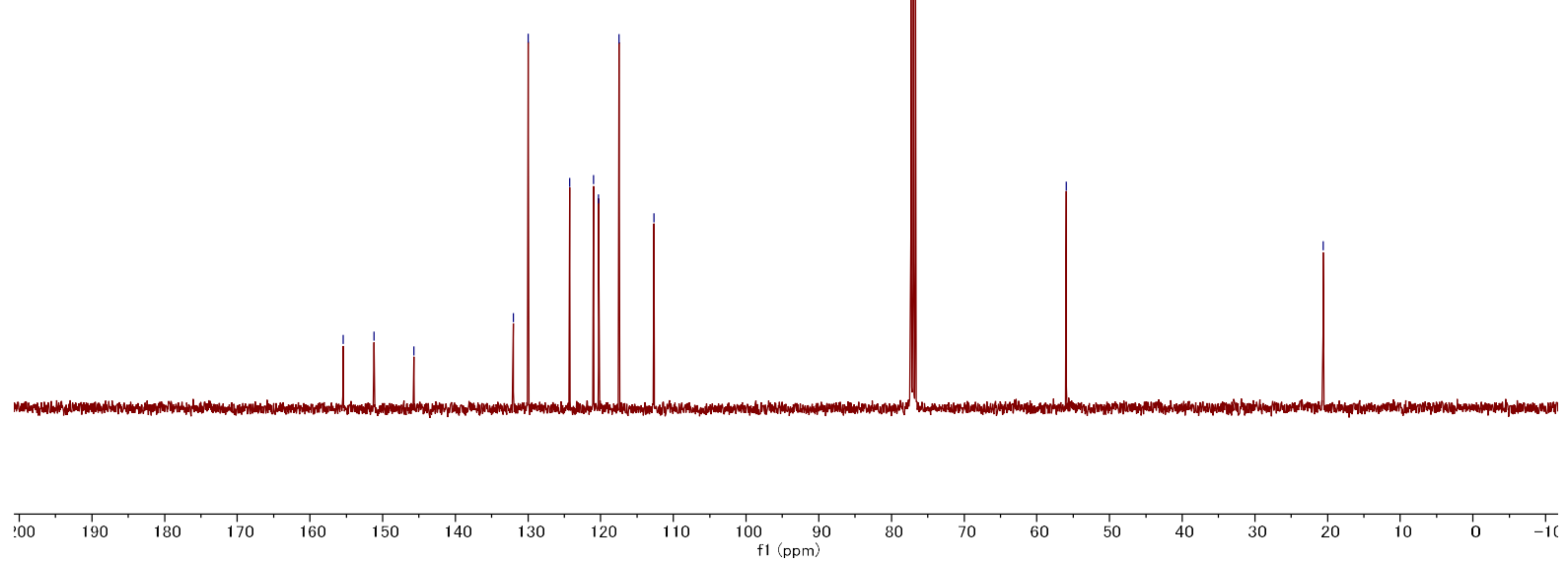

Figure S26. ${ }^{13} \mathrm{C}$ NMR $\left(101 \mathrm{MHz}, \mathrm{CDCl}_{3}\right)$ of 1-methoxy-2-( $p$-tolyloxy)benzene (3d).

S29 


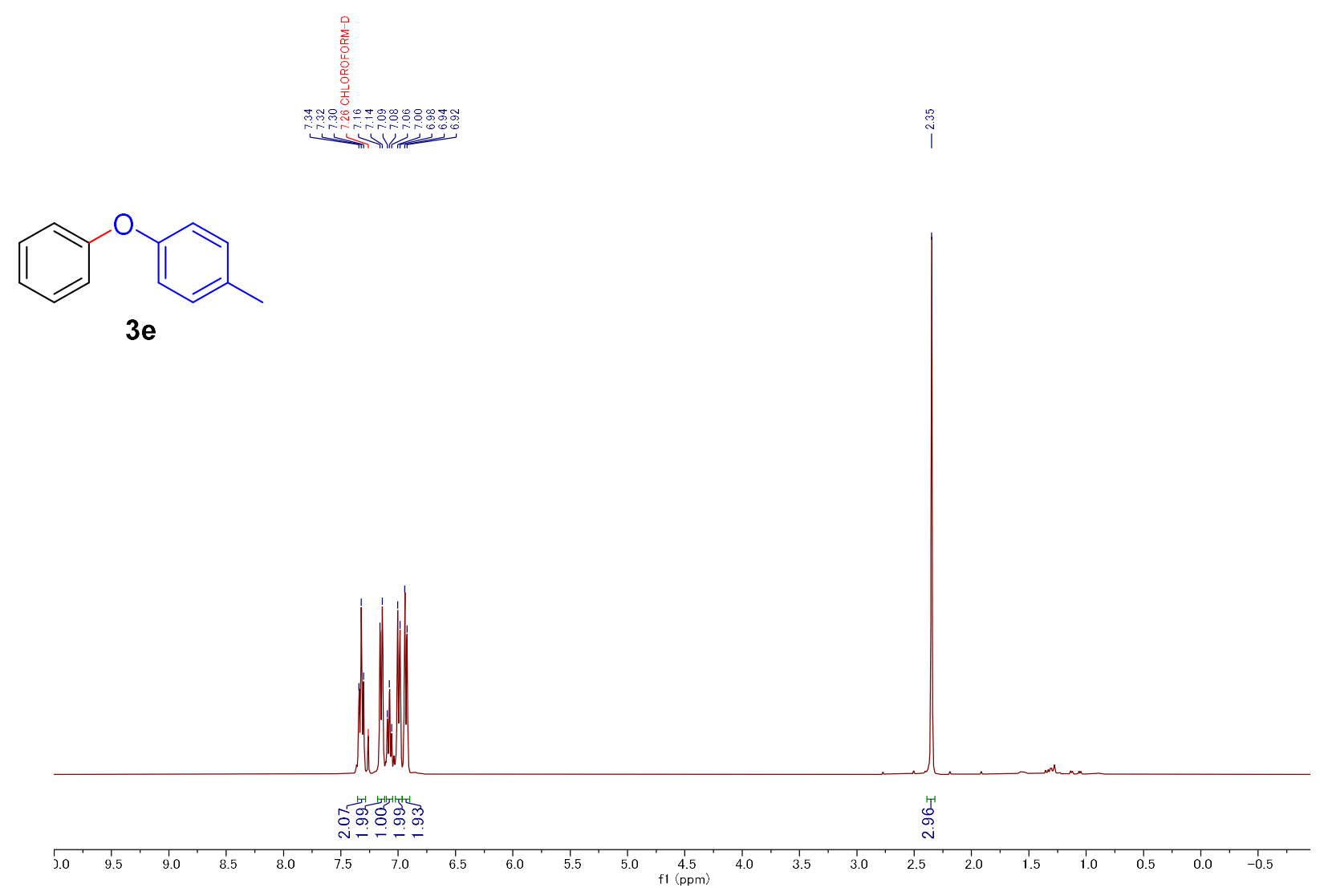

Figure S27. ${ }^{1} \mathrm{H}$ NMR (400 MHz, $\left.\mathrm{CDCl}_{3}\right)$ of 1-methyl-4-phenoxybenzene (3e).

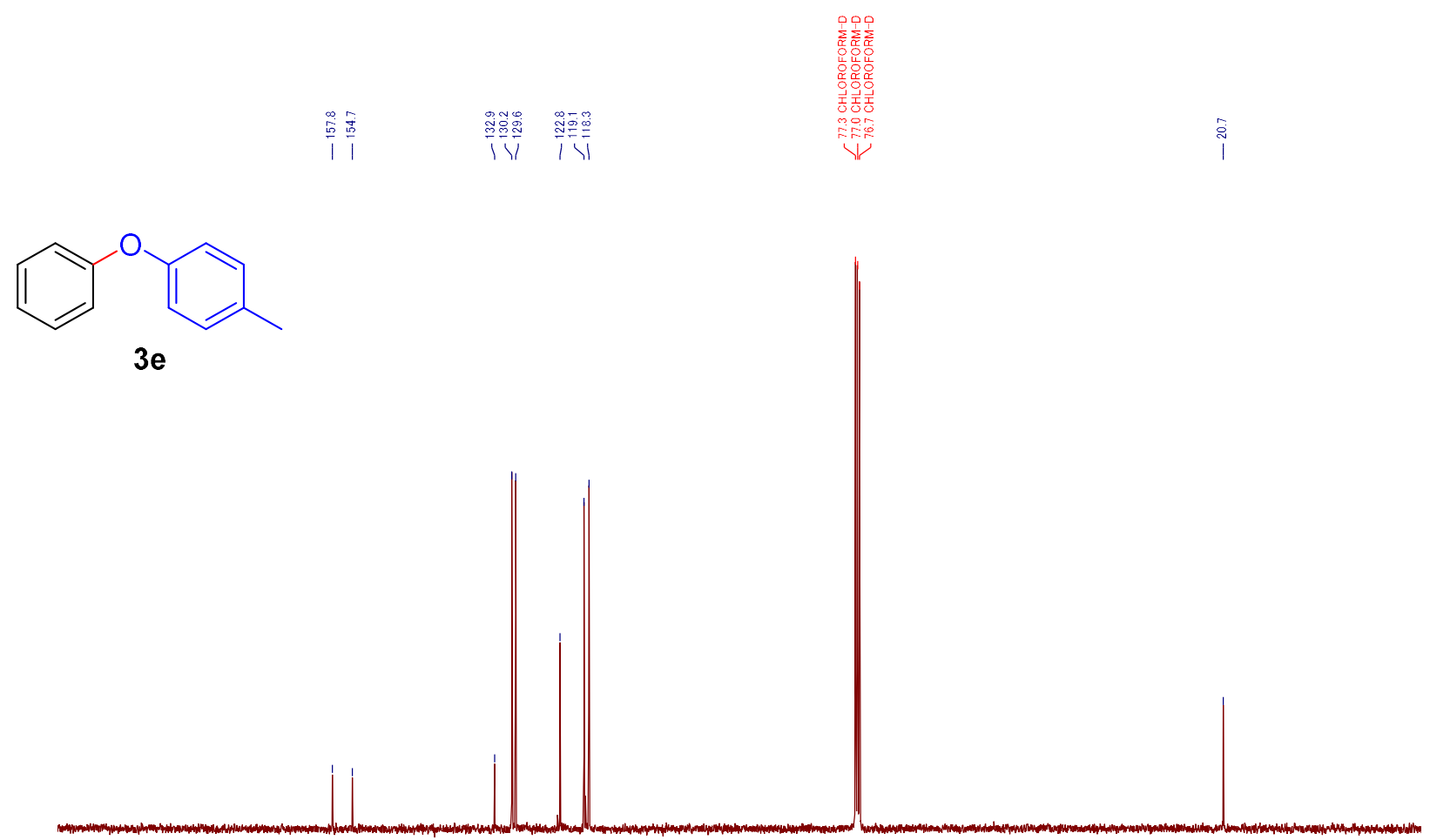

$\begin{array}{lllllllllll}00 & 190 & 180 & 170 & 160 & 150 & 140 & 130 & 120 & 110 & \underset{\mathrm{f} 1(\mathrm{ppm})}{1} 90\end{array}$

Figure S28. ${ }^{13} \mathrm{C}$ NMR (101 MHz, $\left.\mathrm{CDCl}_{3}\right)$ of 1-methyl-4-phenoxybenzene (3e). 


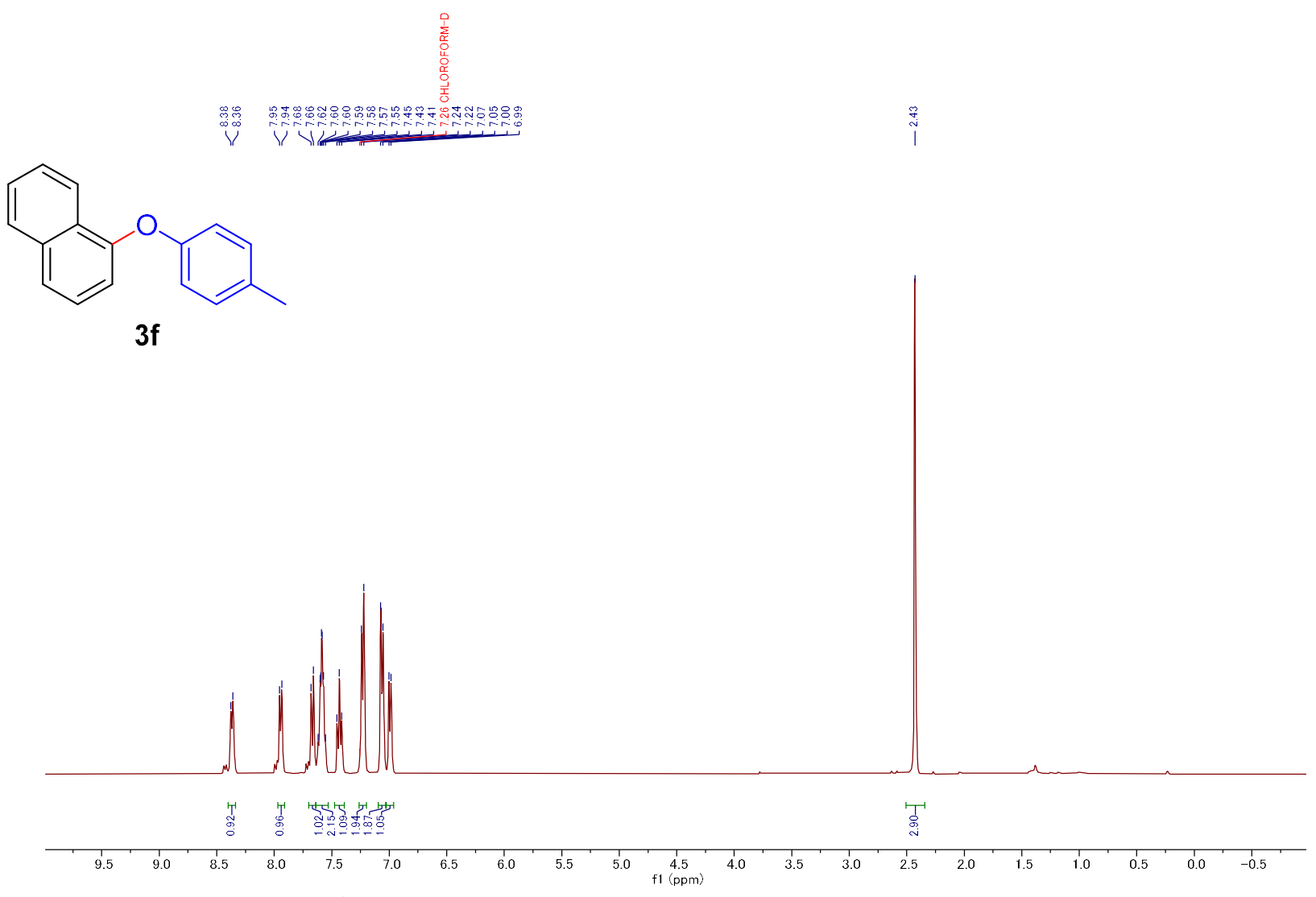

Figure S29. ${ }^{1} \mathrm{H}$ NMR (400 MHz, $\left.\mathrm{CDCl}_{3}\right)$ of 1-( $p$-tolyloxy)naphthalene (3f).

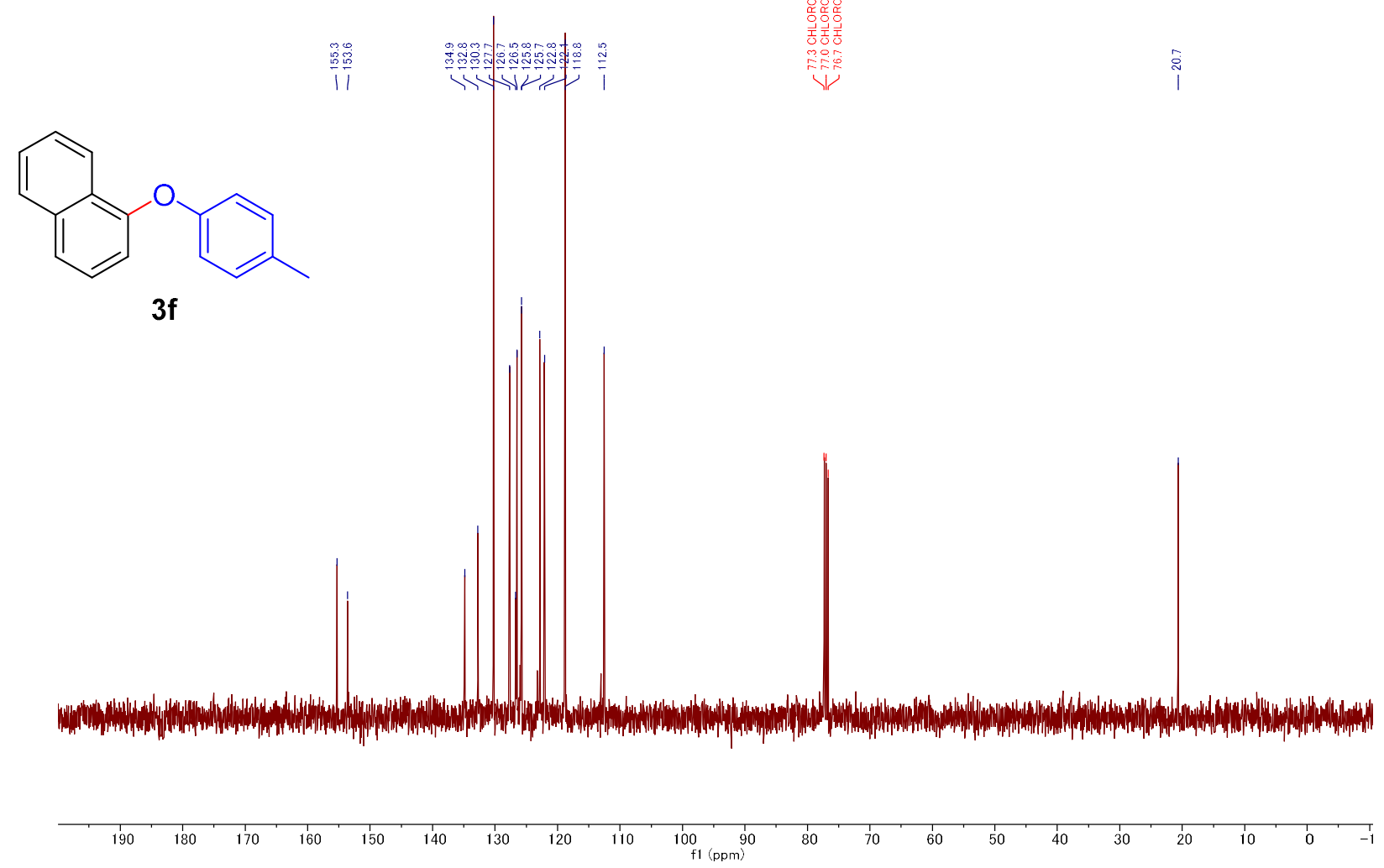

Figure S30. ${ }^{13} \mathrm{C}$ NMR $\left(101 \mathrm{MHz}, \mathrm{CDCl}_{3}\right)$ of 1-(p-tolyloxy)naphthalene (3f). 


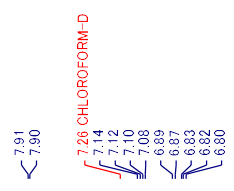<smiles>COc1ncccc1Oc1ccc(C)cc1</smiles>

$3 g$
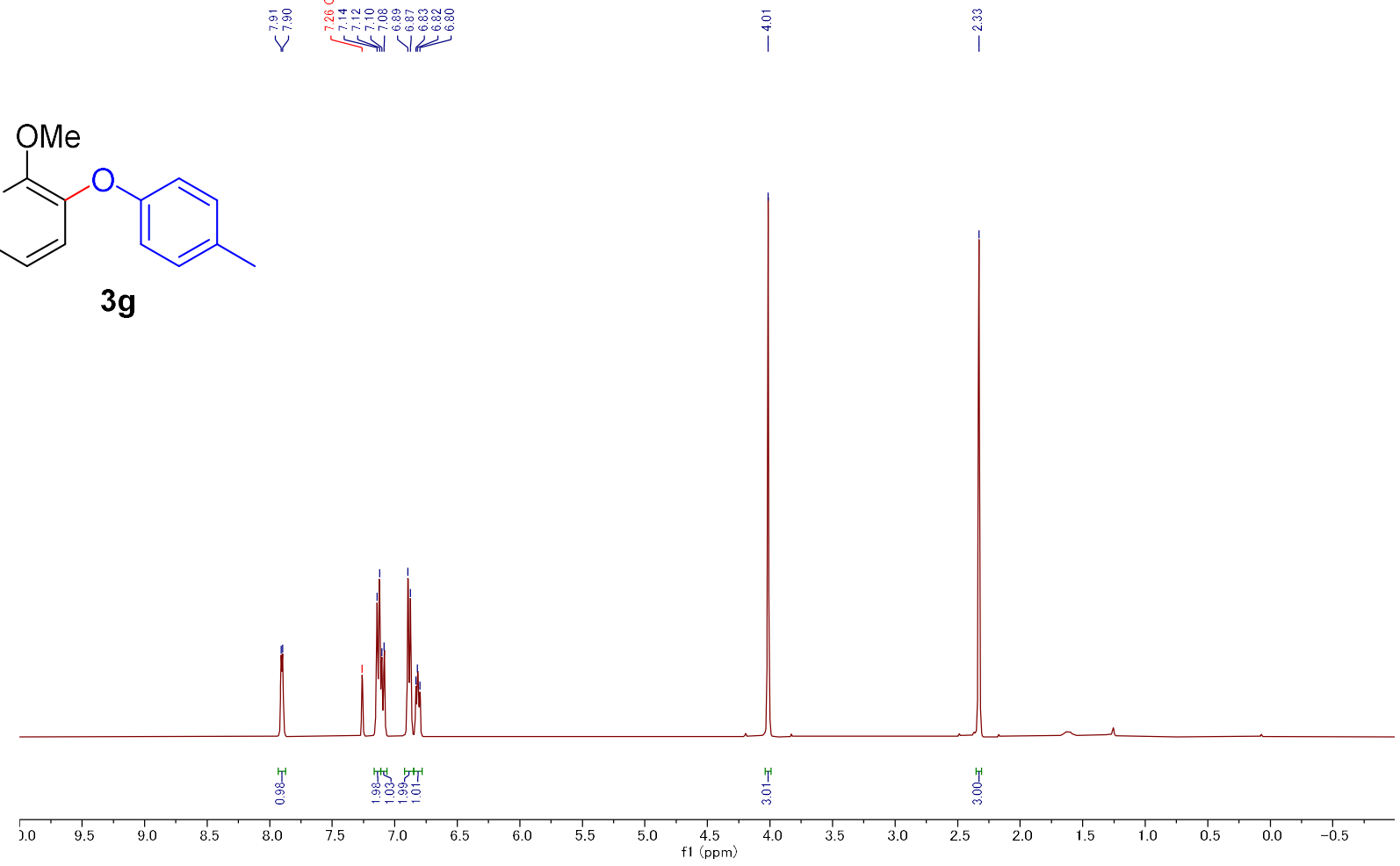

Figure S31. ${ }^{1} \mathrm{H}$ NMR (400 MHz, $\mathrm{CDCl}_{3}$ ) of 2-methoxy-3-(p-tolyloxy)pyridine (3g).

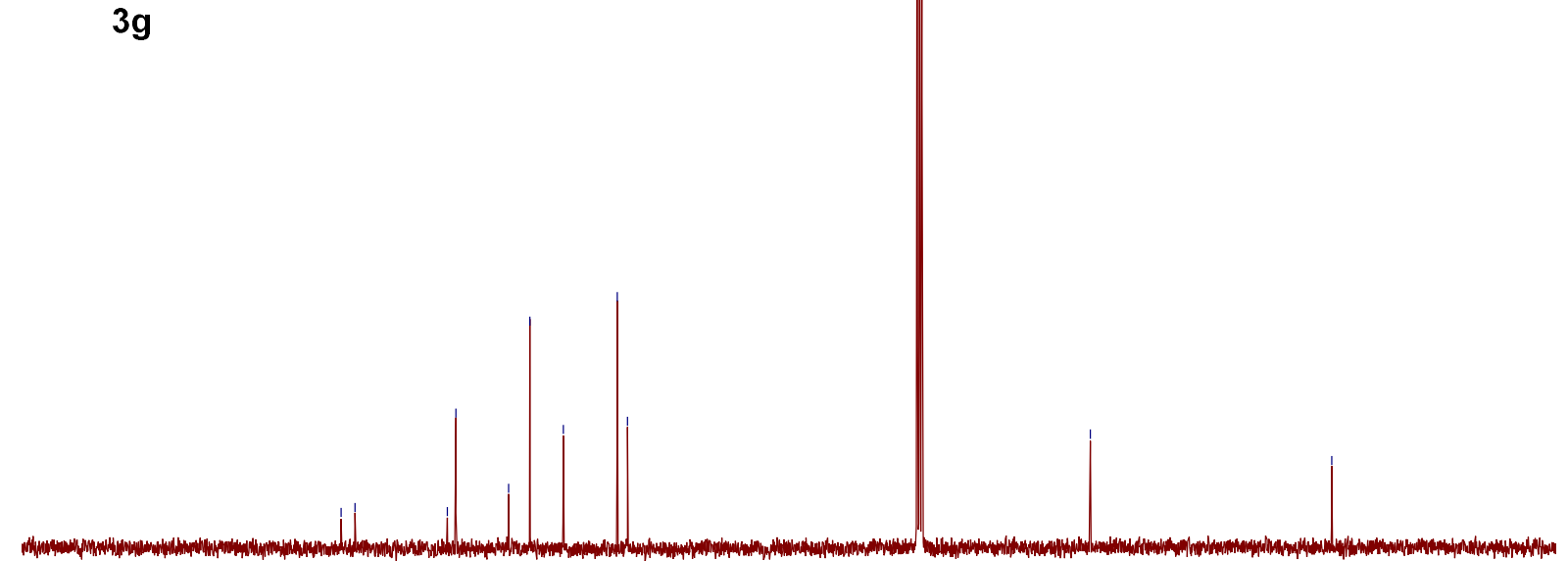<smiles>COc1ncccc1Oc1ccc(C)cc1</smiles>

$3 g$

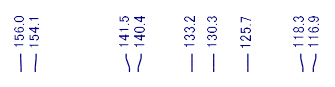

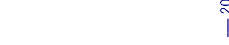

Figure S32. ${ }^{13} \mathrm{C}$ NMR $\left(101 \mathrm{MHz}, \mathrm{CDCl}_{3}\right)$ of 2-methoxy-3-(p-tolyloxy)pyridine (3g). 
<smiles>Cc1ccc(Oc2ccc(C(F)(F)F)cc2)cc1</smiles>

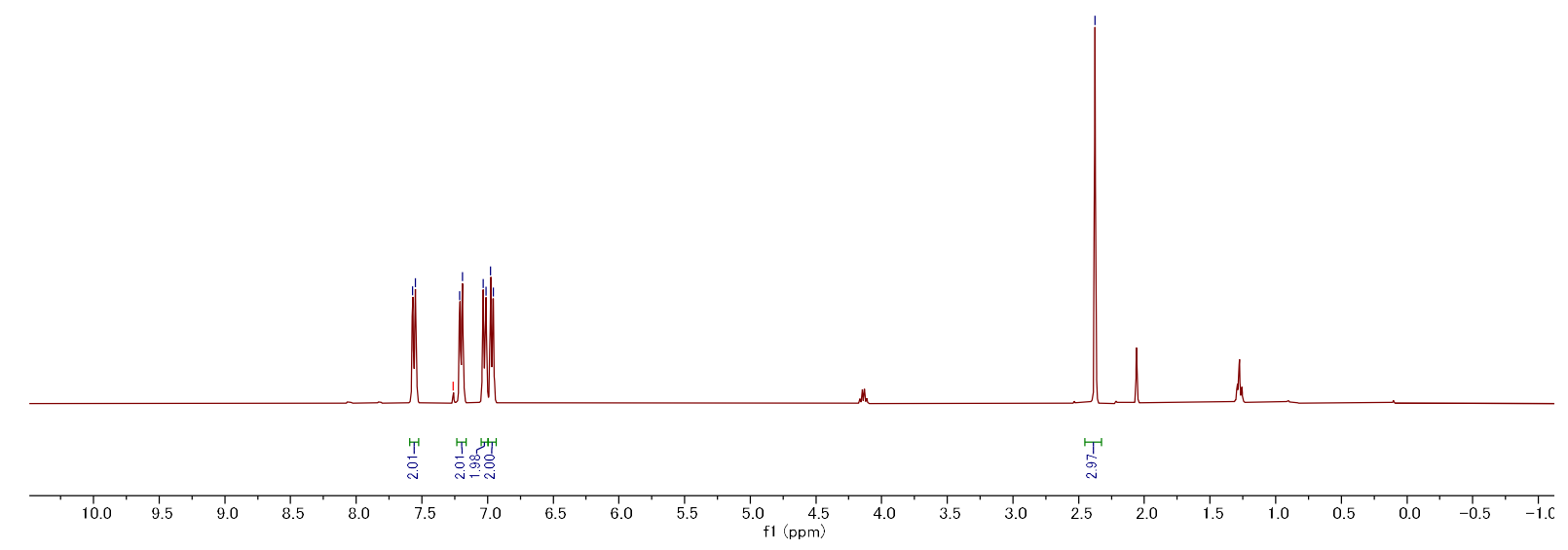

Figure S33. ${ }^{1} \mathrm{H}$ NMR (400 MHz, $\mathrm{CDCl}_{3}$ ) of 1-methyl-4-(4-trifluoromethylphenoxy)benzene (3h).
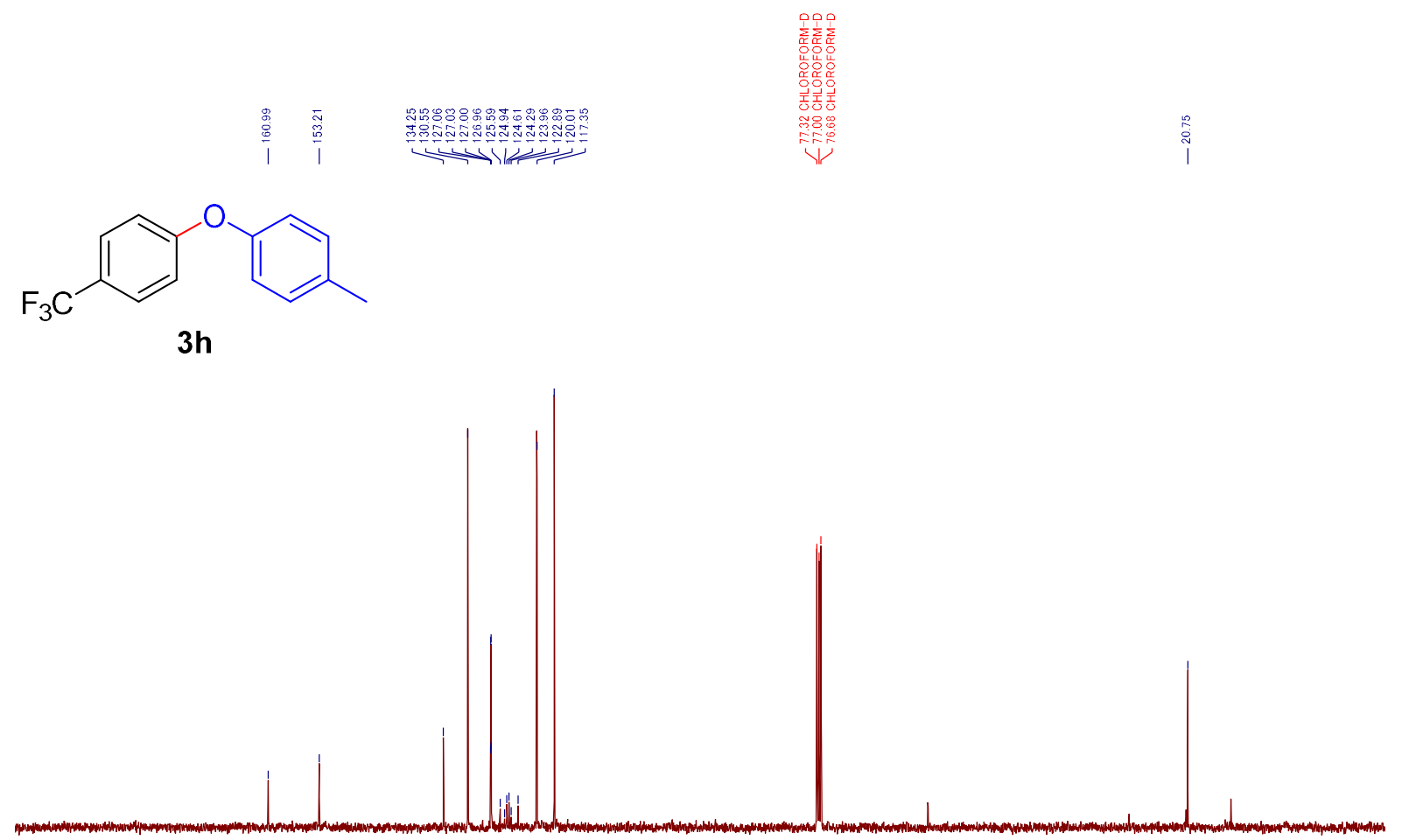

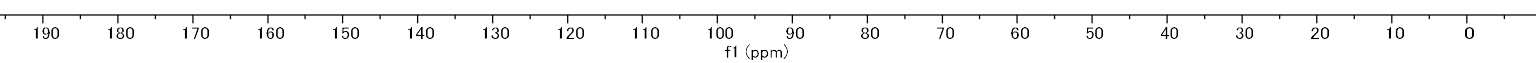

Figure S34. ${ }^{13} \mathrm{C}$ NMR (101 MHz, $\left.\mathrm{CDCl}_{3}\right)$ of 1-methyl-4-(4-trifluoromethylphenoxy)benzene (3h). 
<smiles>Cc1ccc(Oc2ccc(C(F)(F)F)cc2)cc1</smiles>

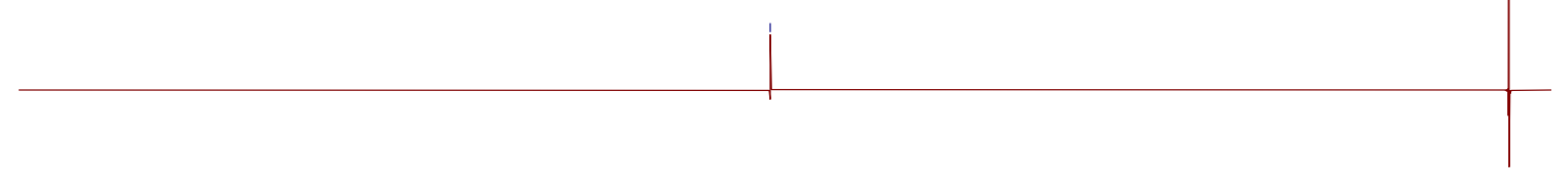

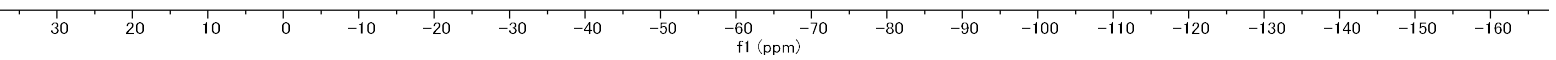

Figure S35. ${ }^{19} \mathrm{~F}$ NMR (376 MHz, $\mathrm{CDCl}_{3}$ ) of 1-methyl-4-(4-trifluoromethylphenoxy)benzene (3h).

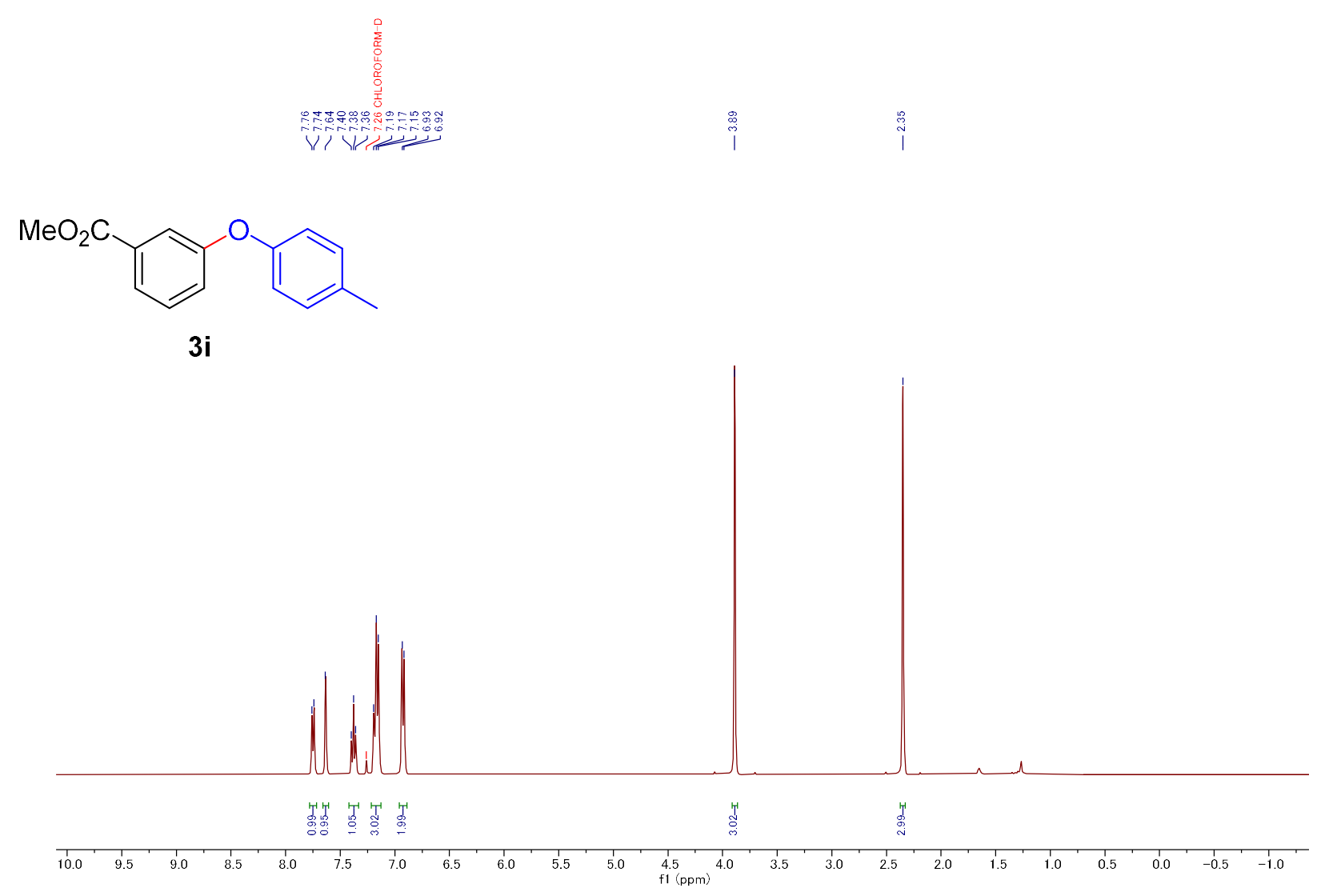

Figure S36. ${ }^{1} \mathrm{H}$ NMR (400 MHz, $\left.\mathrm{CDCl}_{3}\right)$ of methyl 3-(p-tolyloxy)benzoate (3i). 

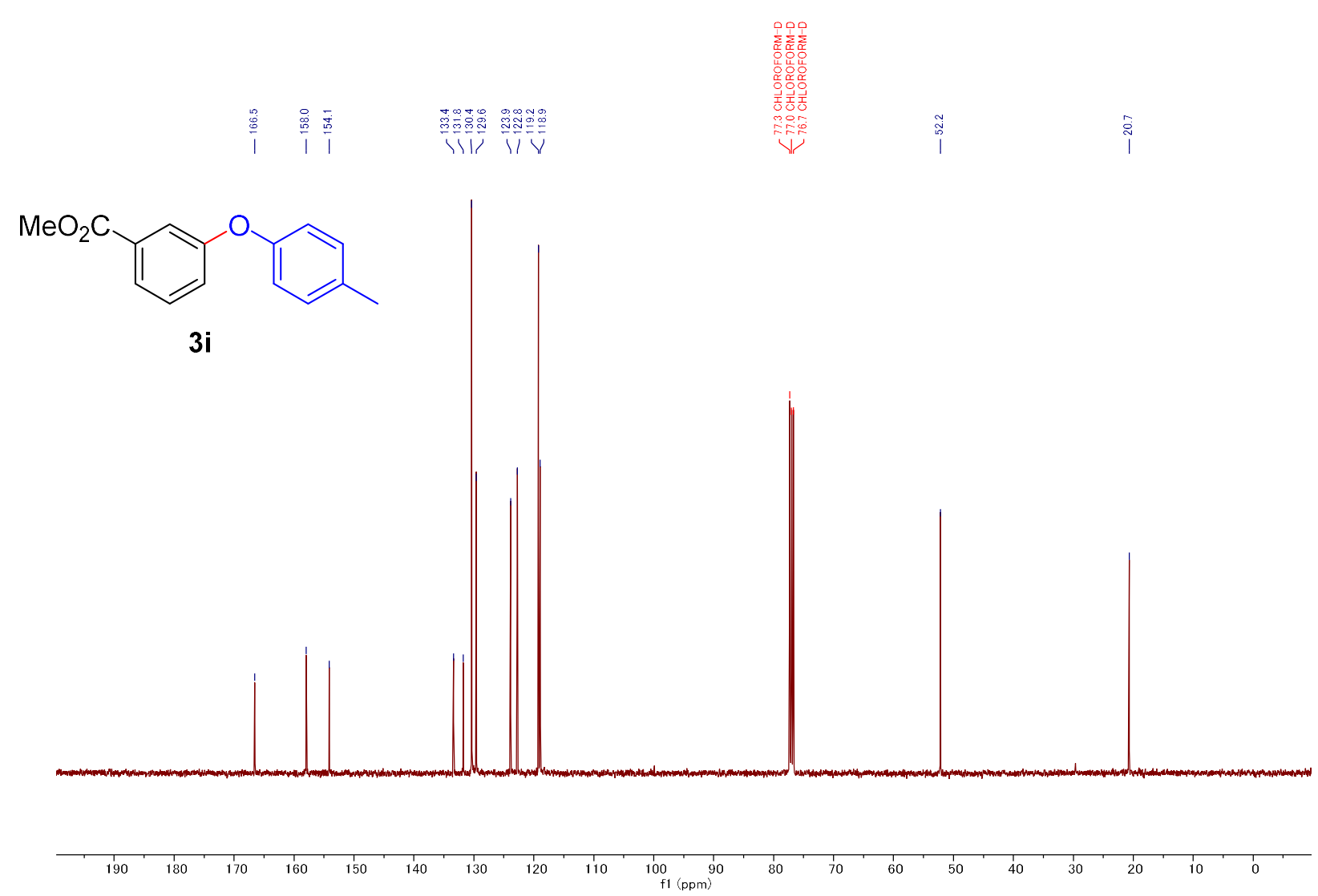

Figure S37. ${ }^{13} \mathrm{C} \mathrm{NMR}\left(101 \mathrm{MHz}, \mathrm{CDCl}_{3}\right)$ of methyl 3-( $p$-tolyloxy)benzoate (3i).

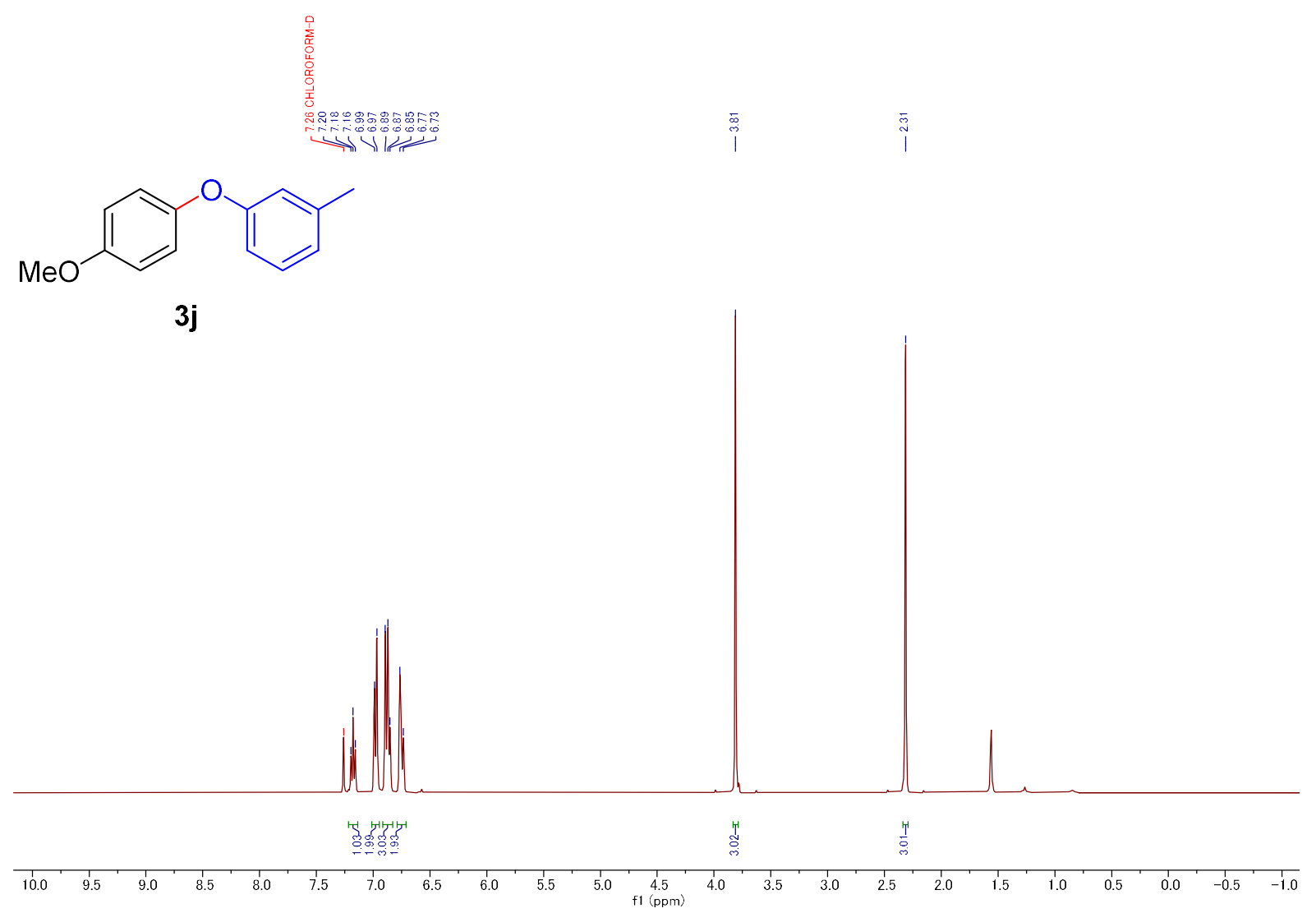

Figure S38. ${ }^{1} \mathrm{H}$ NMR (400 MHz, $\mathrm{CDCl}_{3}$ ) of 1-(4-methoxyphenoxy)-3-methylbenzene (3j). 

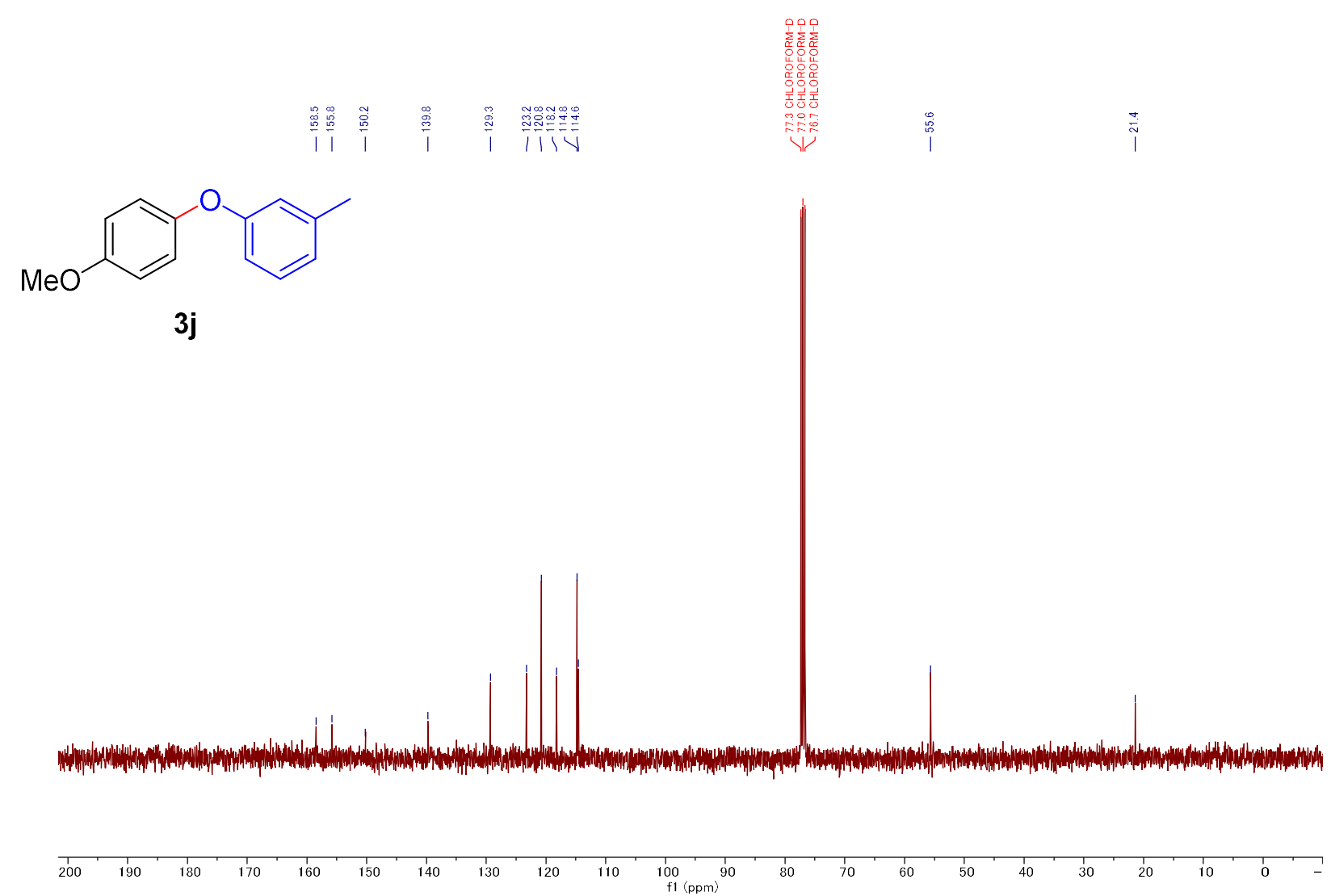

Figure S39. ${ }^{13} \mathrm{C}$ NMR (101 MHz, $\left.\mathrm{CDCl}_{3}\right)$ of 1-(4-methoxyphenoxy)-3-methylbenzene (3j).

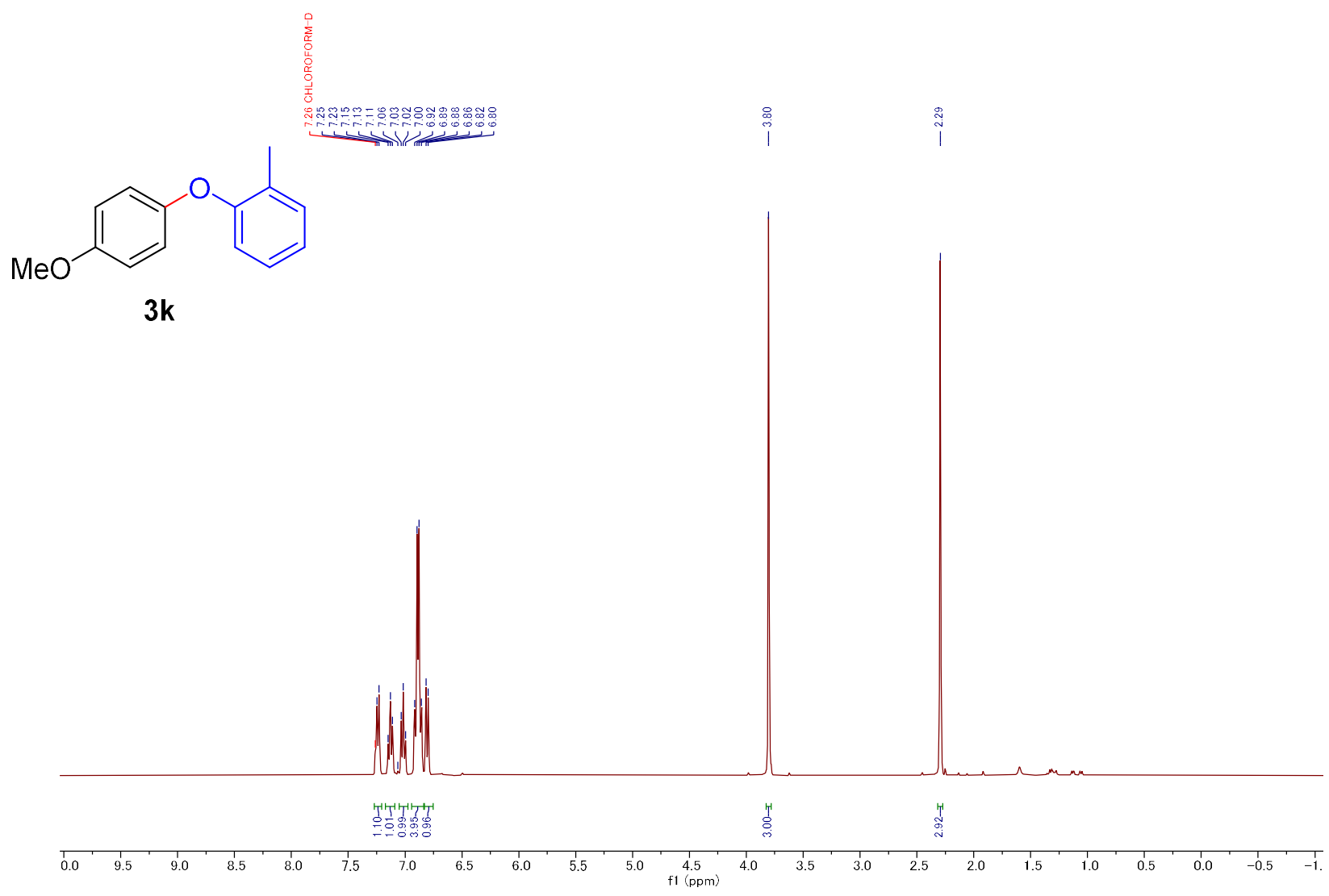

Figure S40. ${ }^{1} \mathrm{H}$ NMR (400 MHz, $\left.\mathrm{CDCl}_{3}\right)$ of 1-(4-methoxyphenoxy)-2-methylbenzene (3k). 

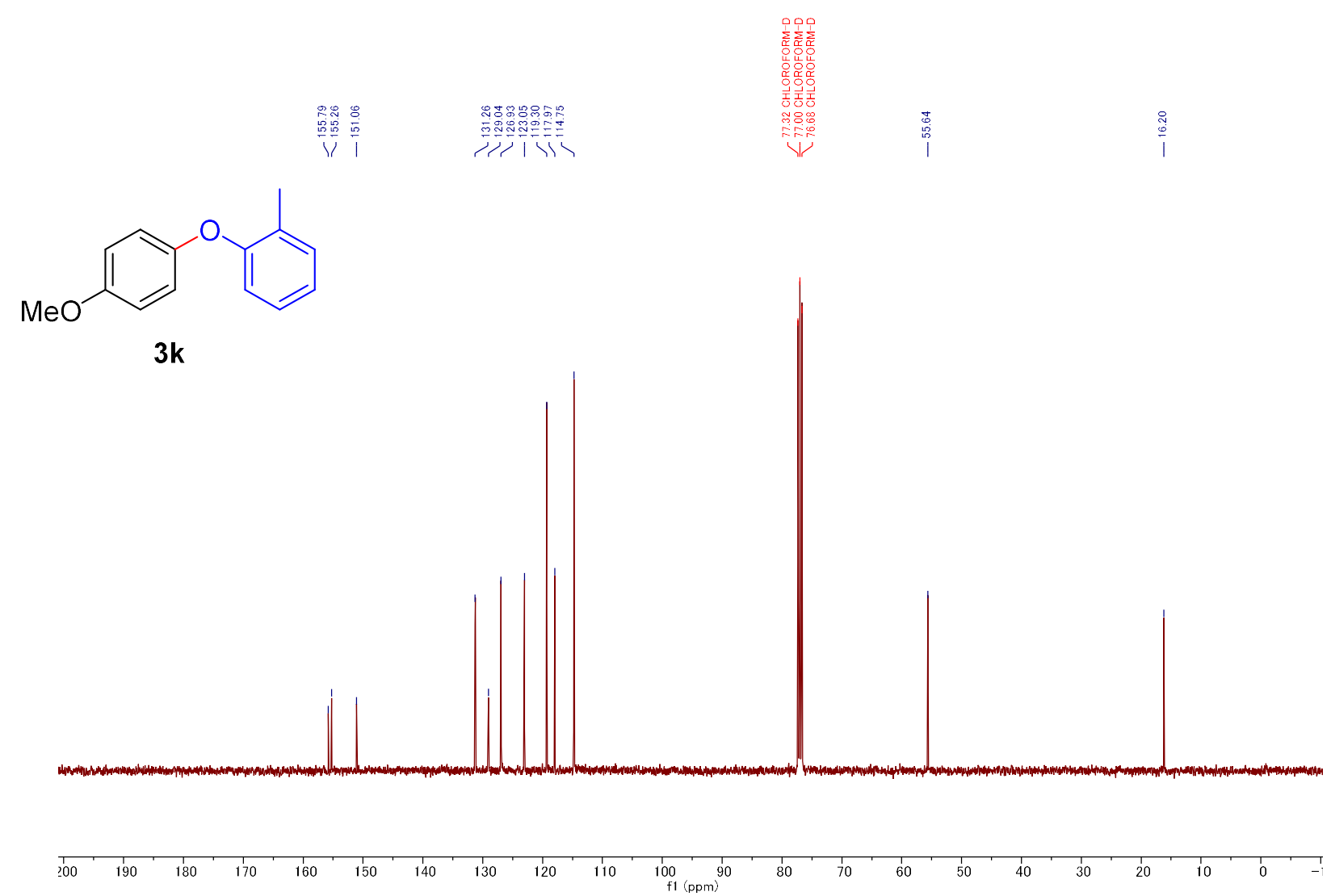

Figure S41. ${ }^{13} \mathrm{C}$ NMR (101 MHz, $\mathrm{CDCl}_{3}$ ) of 1-(4-methoxyphenoxy)-2-methylbenzene (3k).

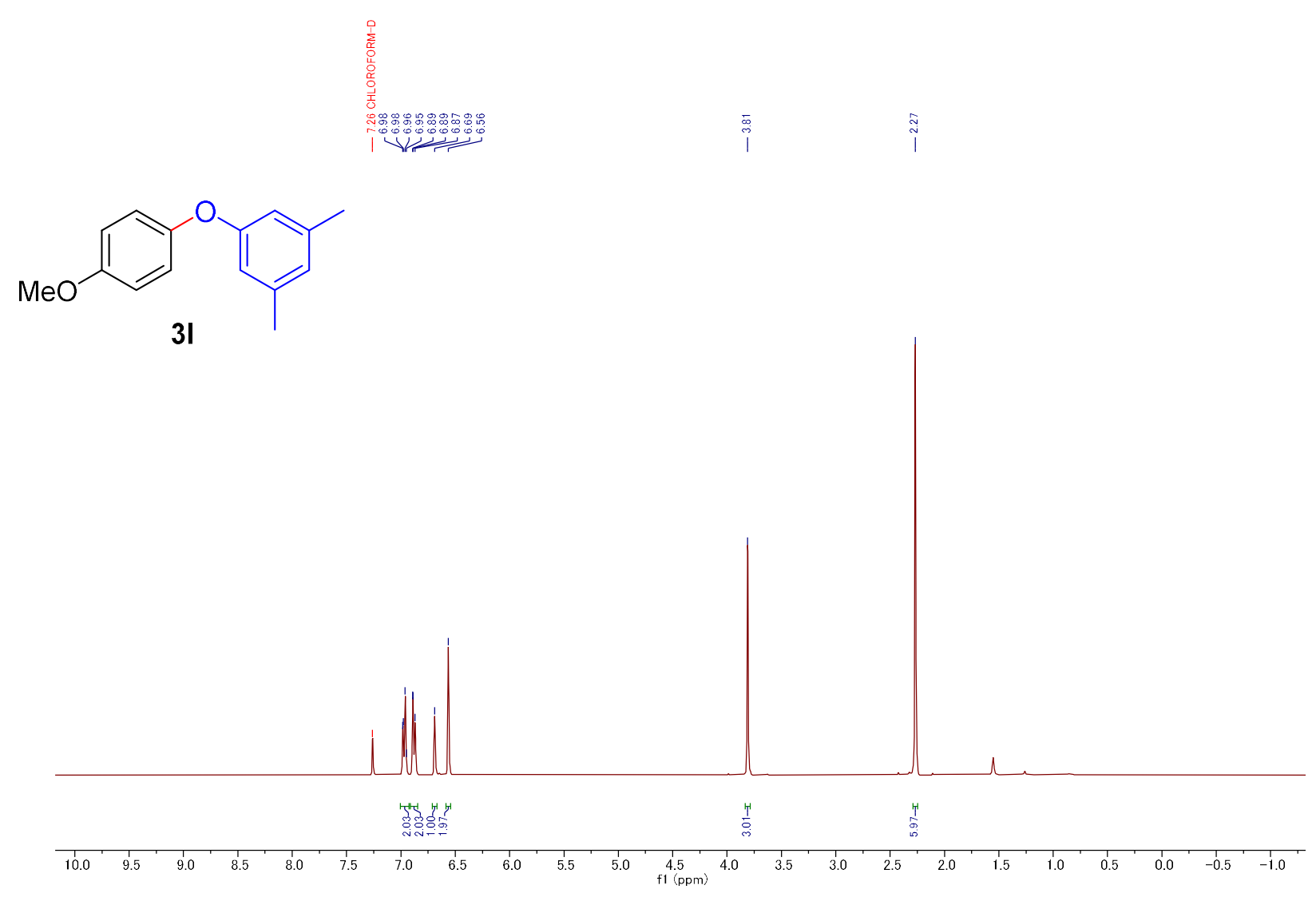

Figure S42. ${ }^{1} \mathrm{H}$ NMR (400 MHz, $\mathrm{CDCl}_{3}$ ) of 1-(4-methoxyphenoxy)-3,5-dimethylbenzene (31). 

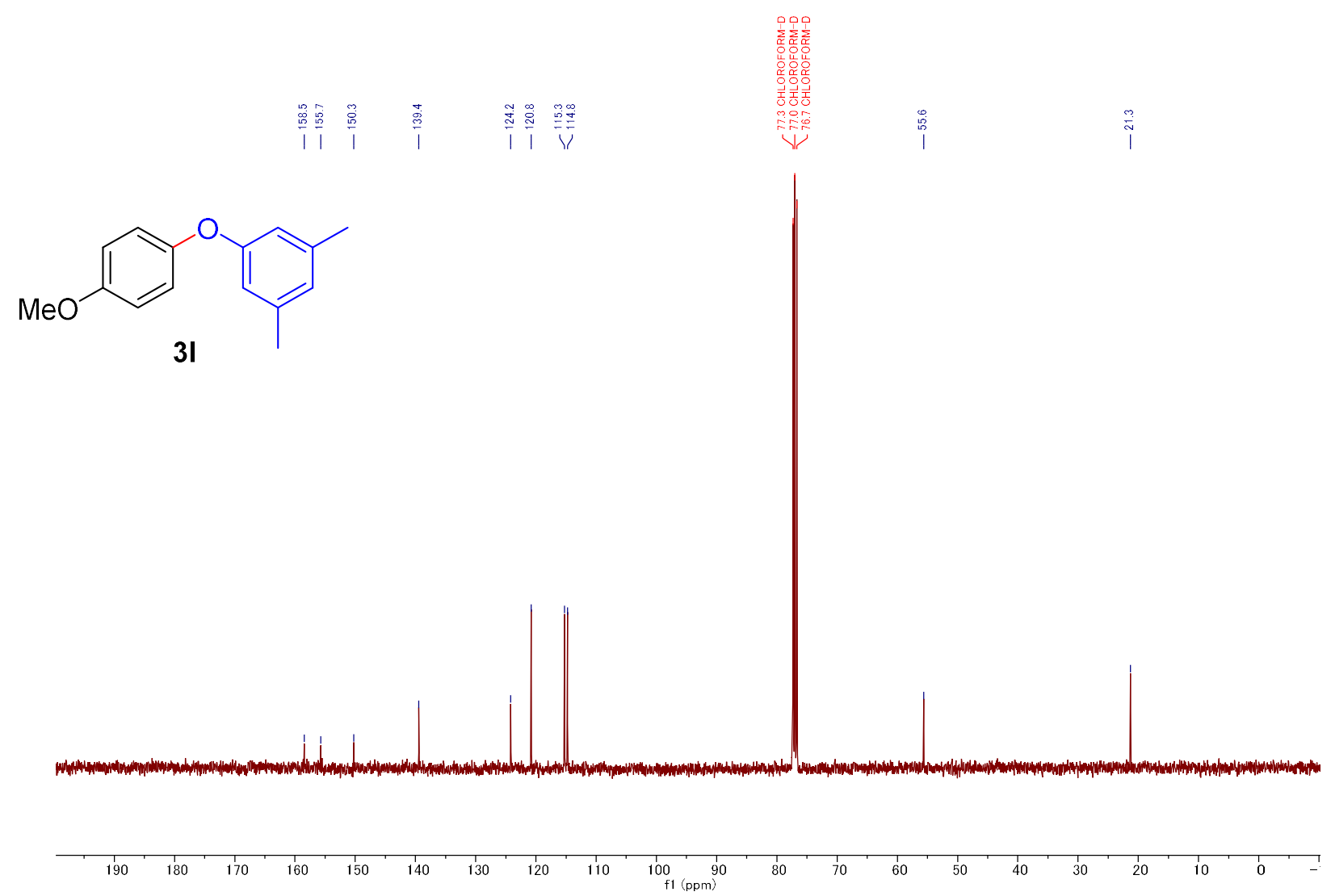

Figure S43. ${ }^{13} \mathrm{C}$ NMR (101 MHz, $\mathrm{CDCl}_{3}$ ) of 1-(4-methoxyphenoxy)-3,5-dimethylbenzene (3I).

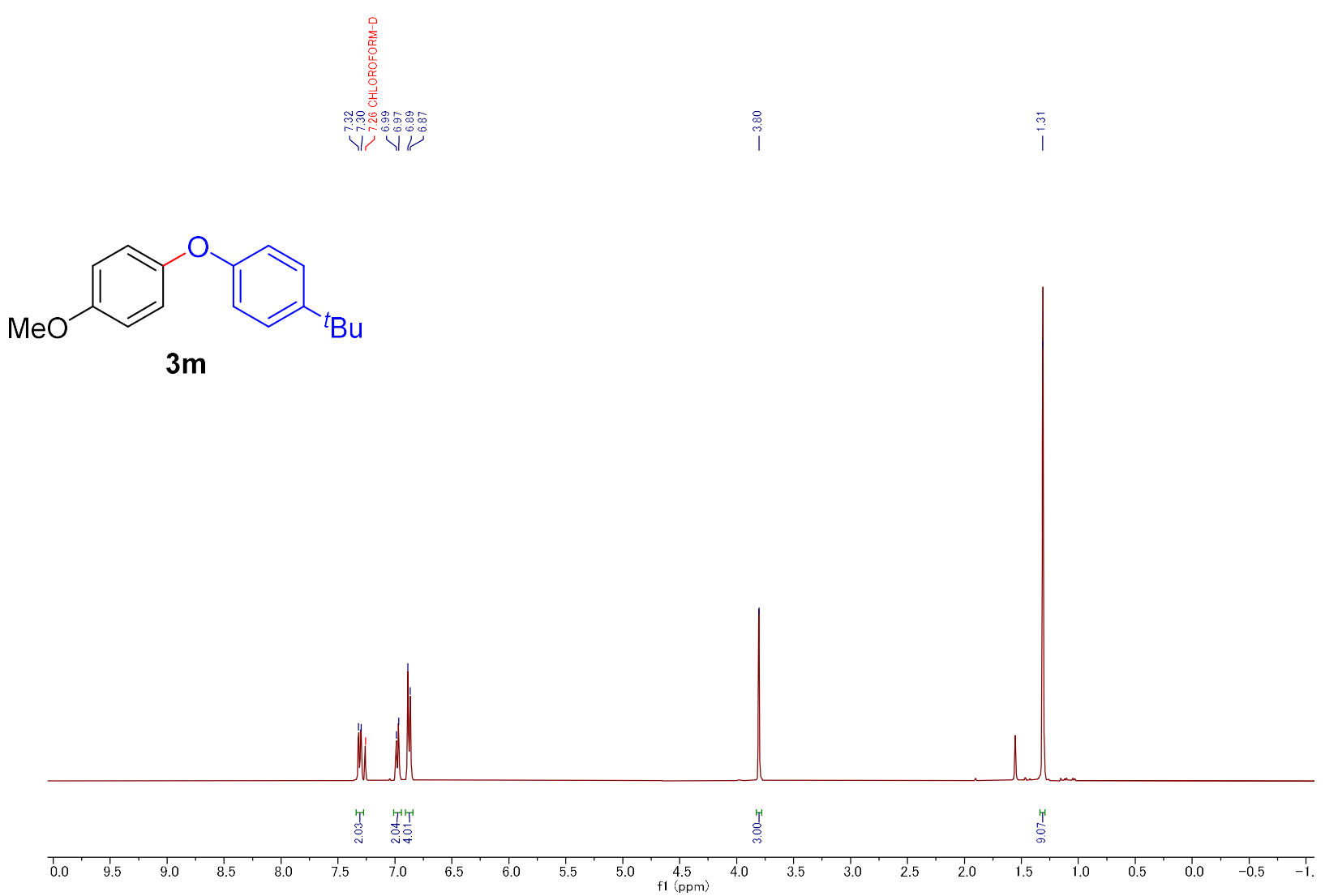

Figure S44. ${ }^{1} \mathrm{H} \mathrm{NMR}\left(400 \mathrm{MHz}, \mathrm{CDCl}_{3}\right)$ of 1-(4-tert-butylphenoxy)-4-methoxybenzene (3m). 

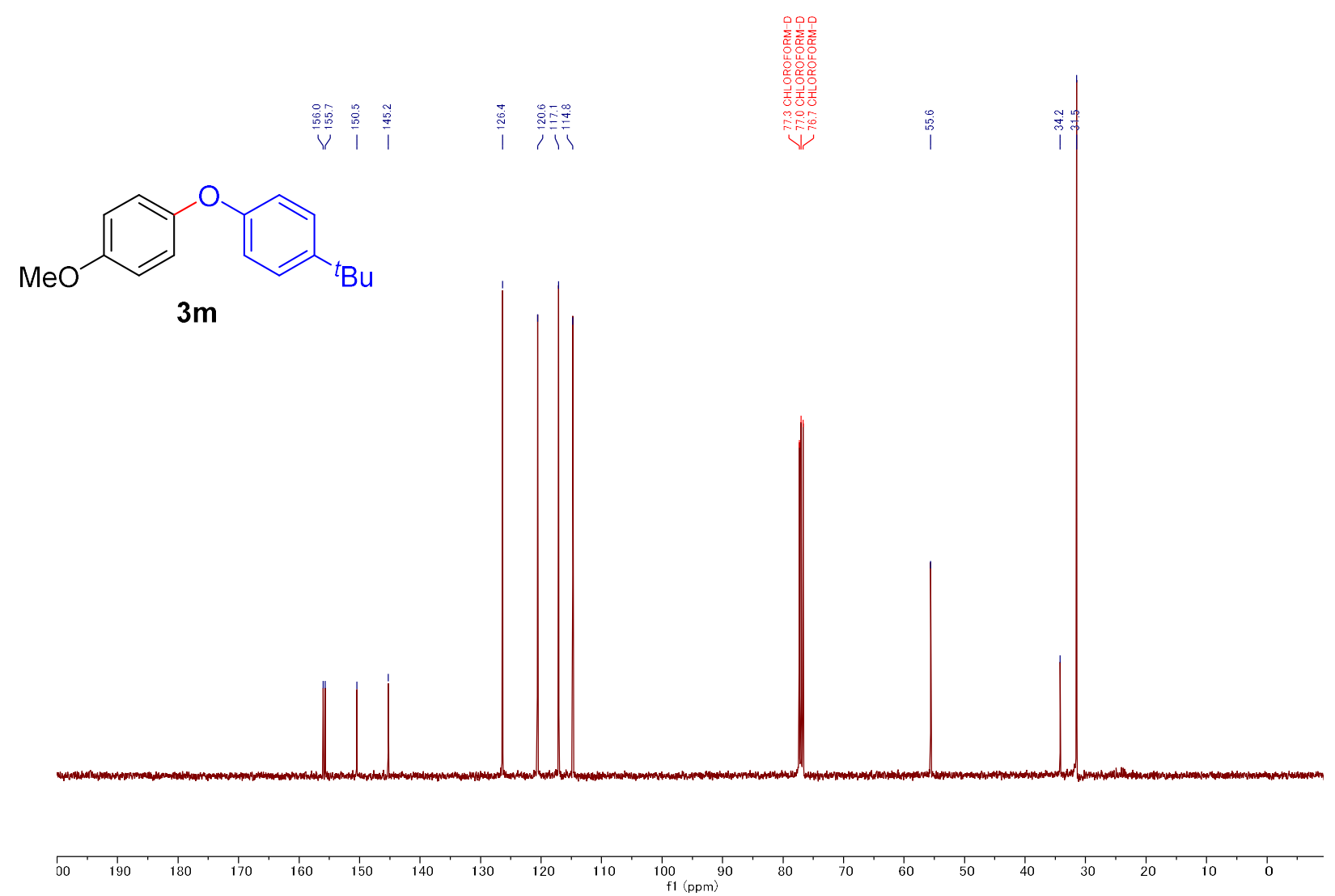

Figure S45. ${ }^{13} \mathrm{C}$ NMR (101 MHz, $\left.\mathrm{CDCl}_{3}\right)$ of 1-(4-tert-butylphenoxy)-4-methoxybenzene (3m).
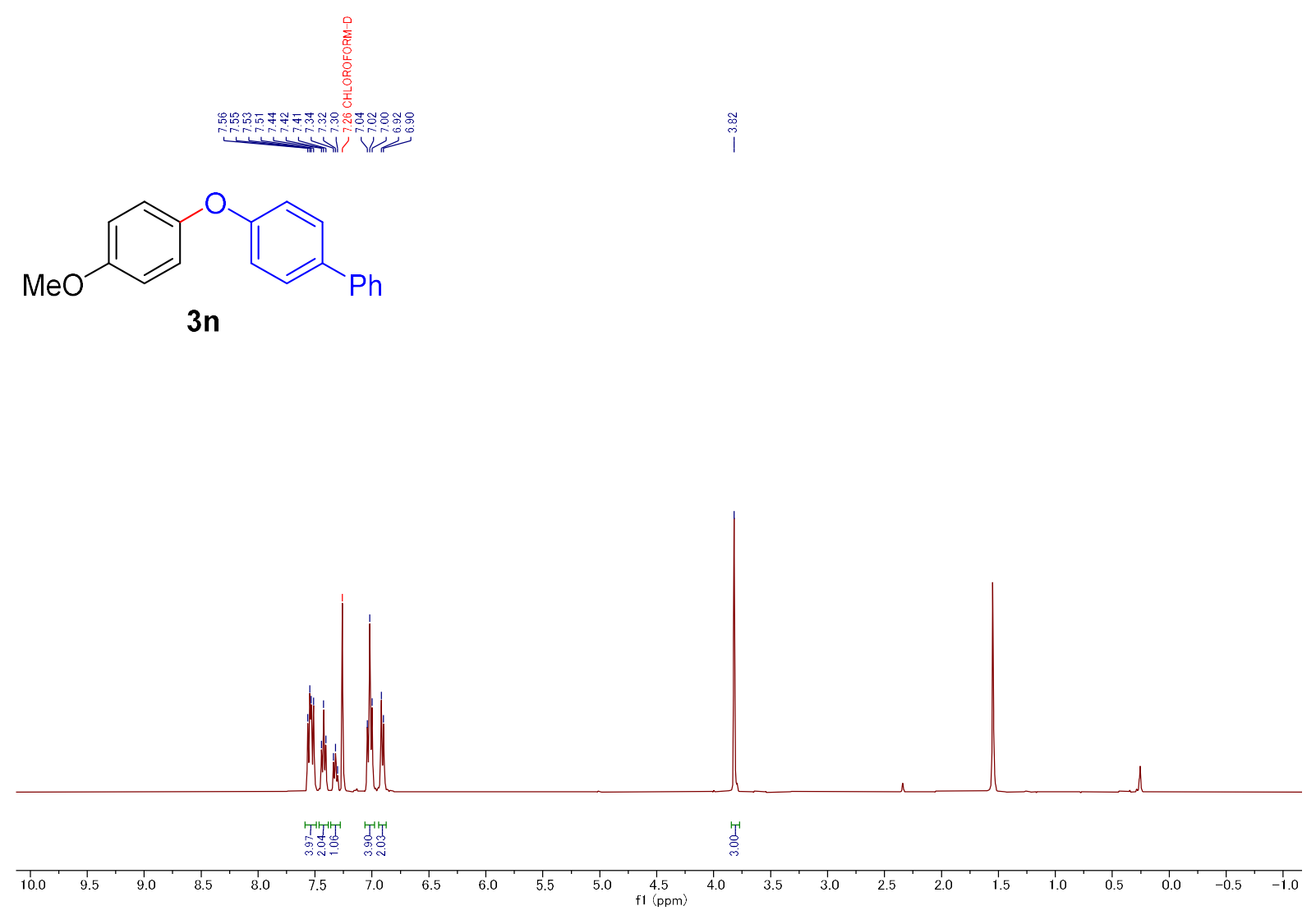

Figure S46. ${ }^{1} \mathrm{H}$ NMR (400 MHz, $\mathrm{CDCl}_{3}$ ) of 4-(4-methoxyphenoxy)-1, 1'-biphenyl (3n). 

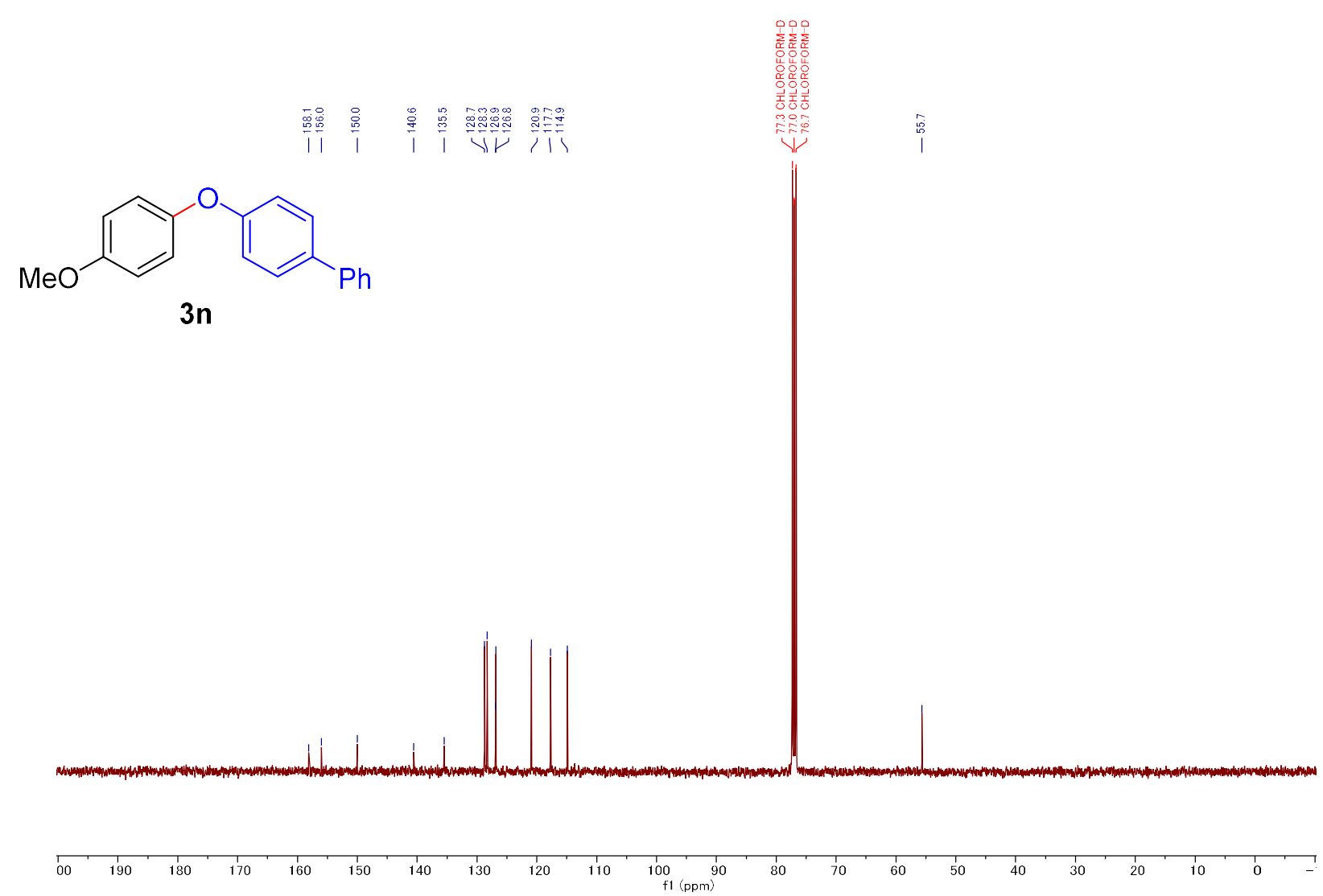

Figure S47. ${ }^{13} \mathrm{C}$ NMR (101 MHz, $\mathrm{CDCl}_{3}$ ) of 4-(4-methoxyphenoxy)-1,1'-biphenyl (3n).

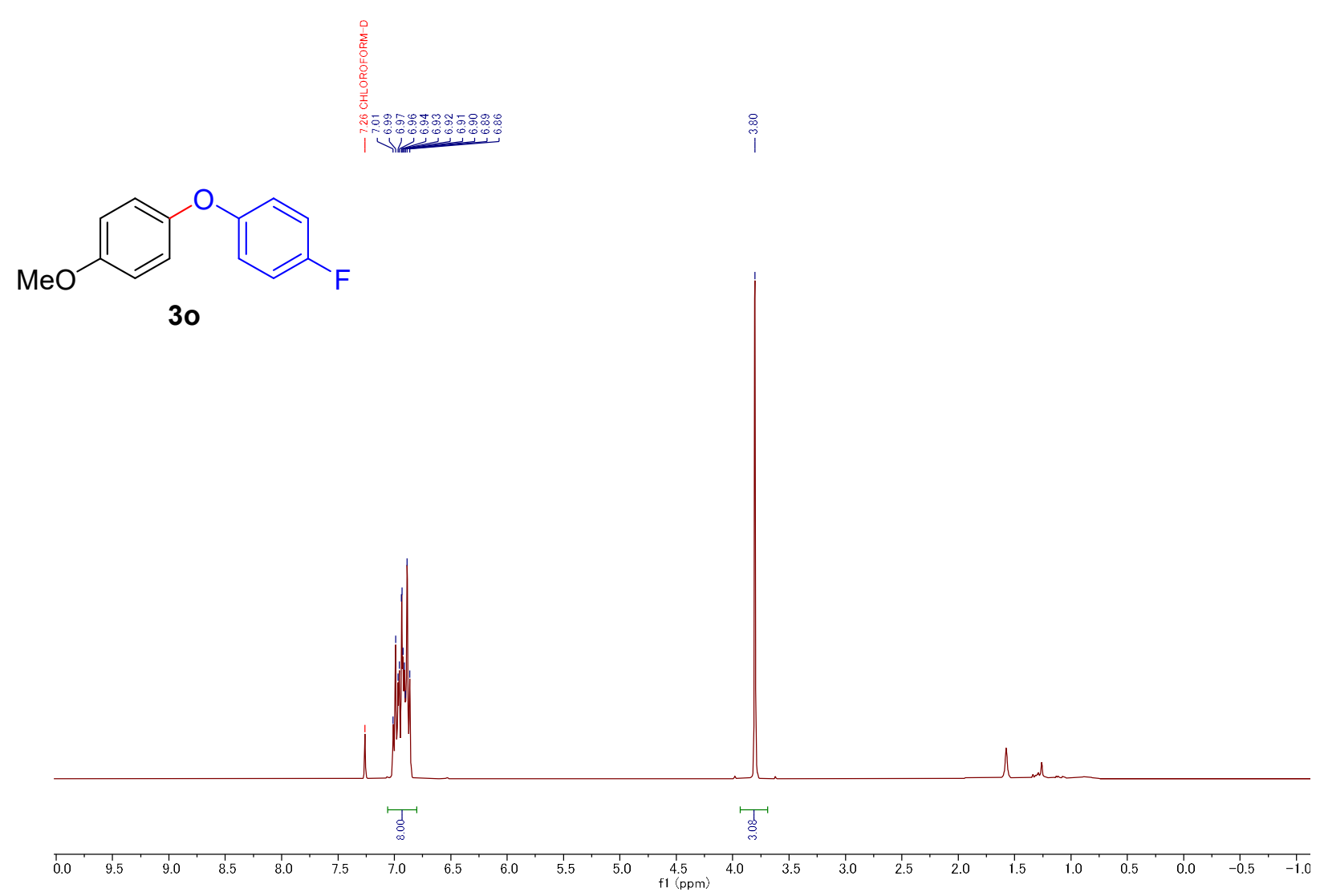

Figure S48. ${ }^{1} \mathrm{H} \mathrm{NMR}\left(400 \mathrm{MHz}, \mathrm{CDCl}_{3}\right.$ ) of 1-fluoro-4-(4-methoxyphenoxy)benzene (3o). 

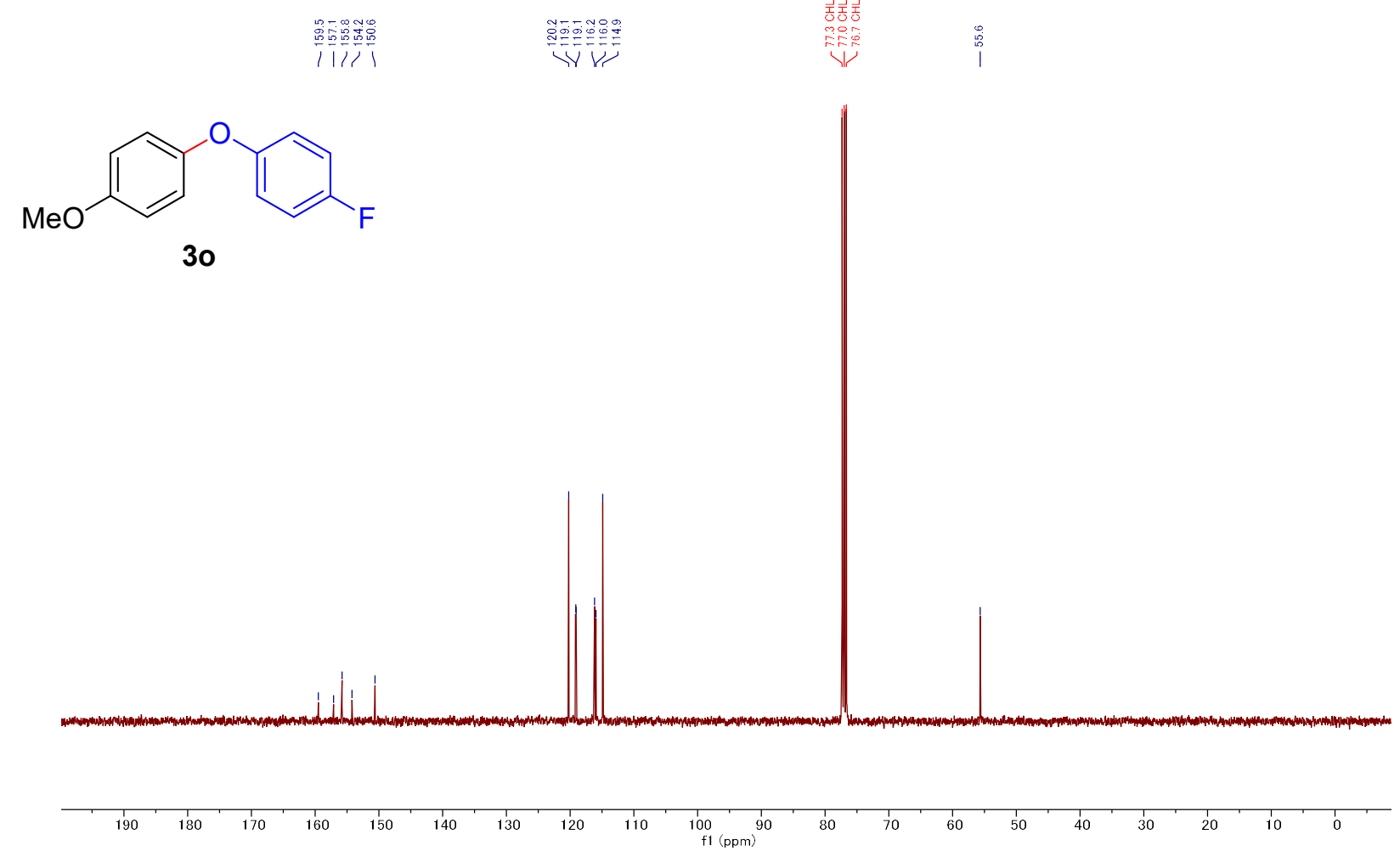

Figure S49. ${ }^{13} \mathrm{C}$ NMR (101 MHz, $\mathrm{CDCl}_{3}$ ) of 1-fluoro-4-(4-methoxyphenoxy)benzene (3o).

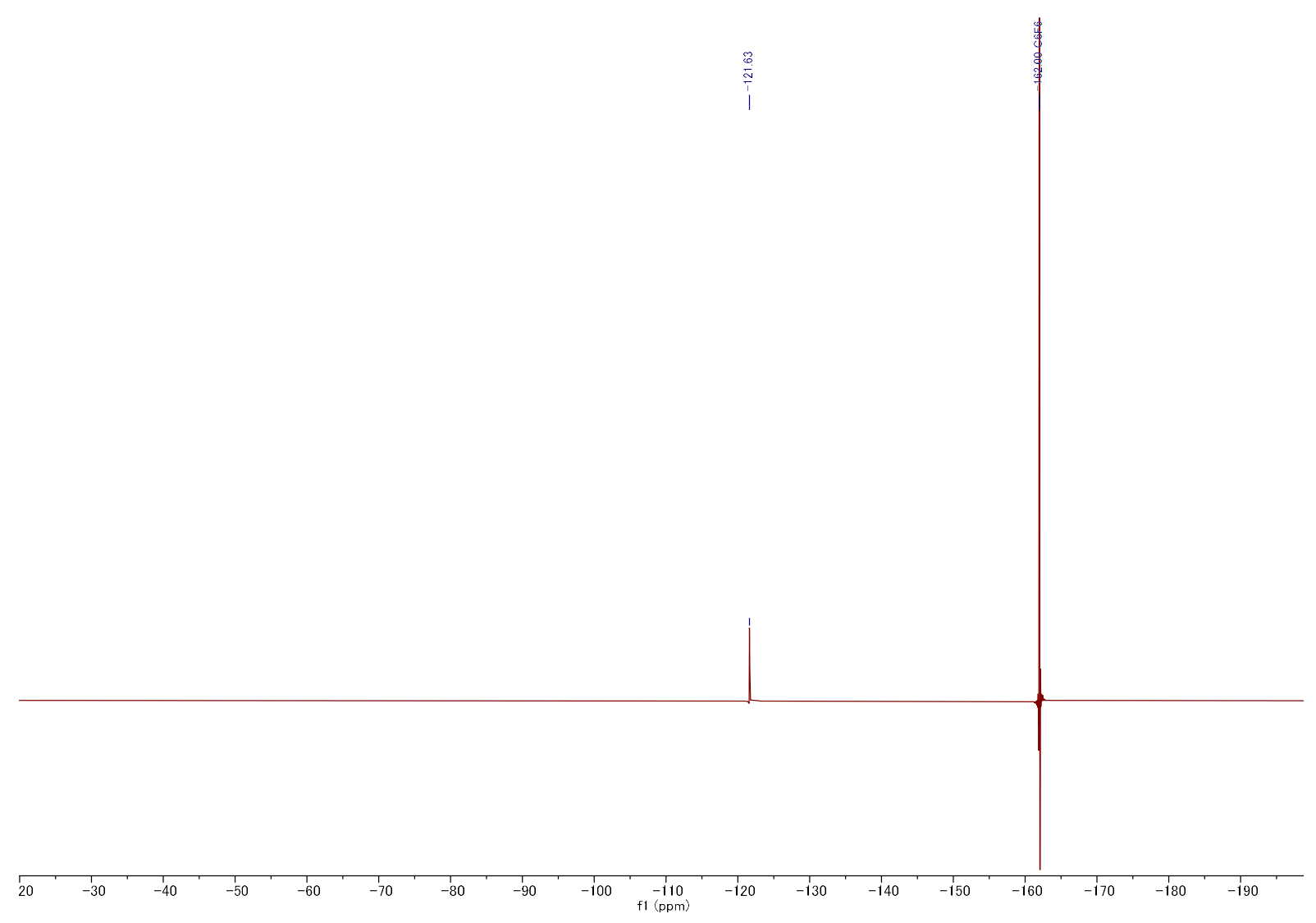

Figure S50. ${ }^{19}$ F NMR (376 MHz, $\mathrm{CDCl}_{3}$ ) of 1-fluoro-4-(4-methoxyphenoxy)benzene (3o). 


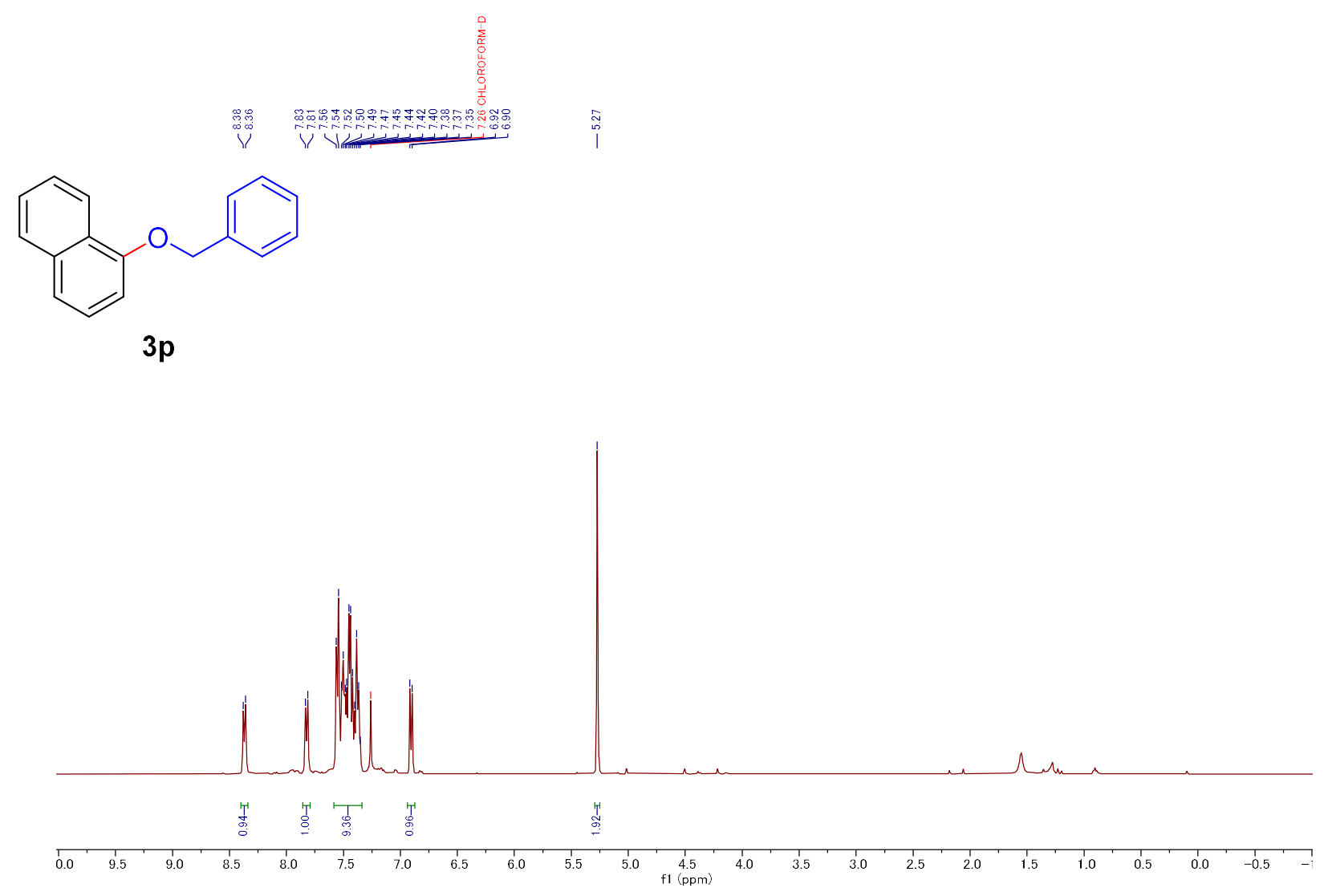

Figure S51. ${ }^{1} \mathrm{H}$ NMR (400 MHz, $\left.\mathrm{CDCl}_{3}\right)$ of 1-(benzyloxy)naphthalene (3p).
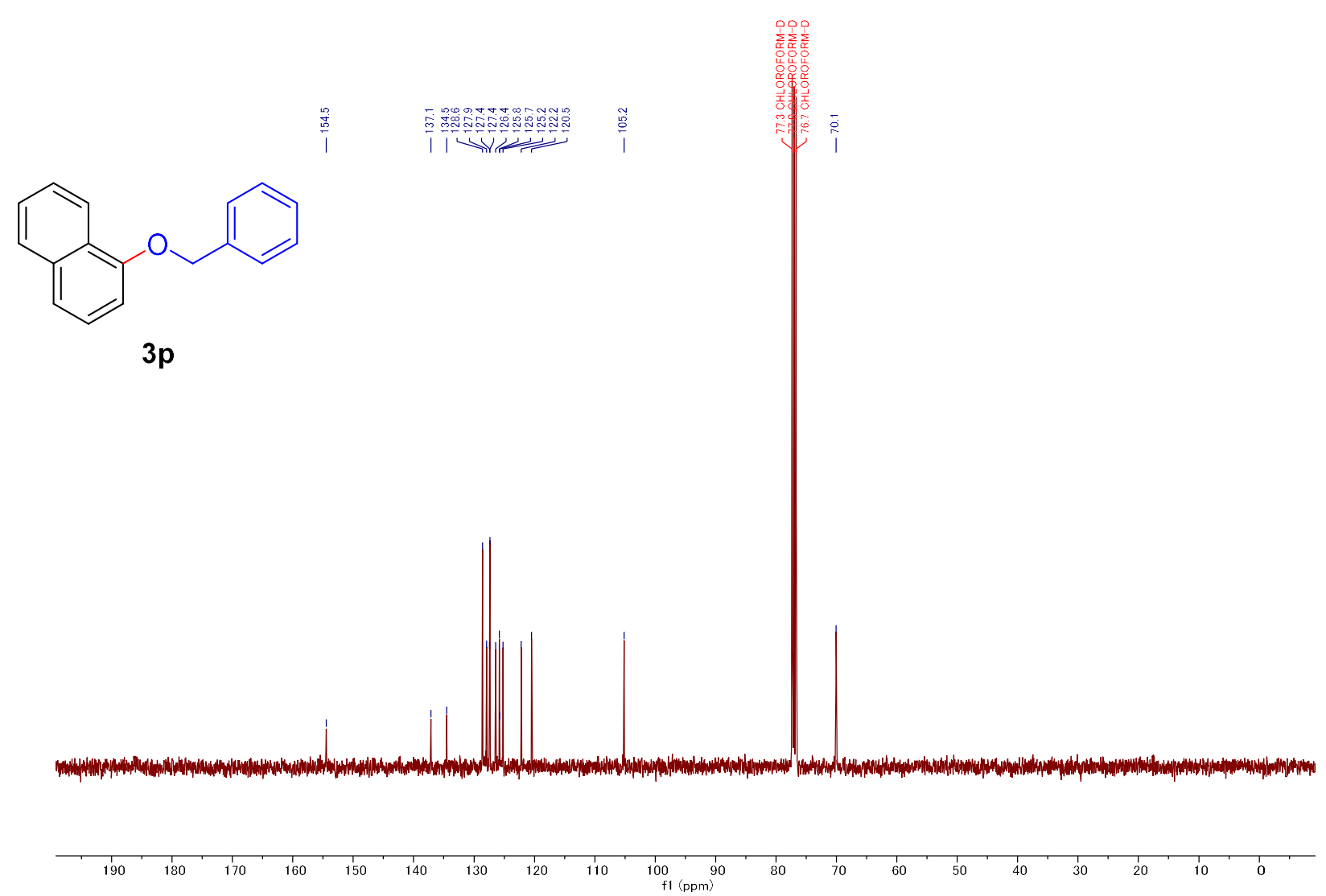

Figure S52. ${ }^{13} \mathrm{C}$ NMR (101 MHz, $\left.\mathrm{CDCl}_{3}\right)$ of 1-(benzyloxy)naphthalene (3p). 


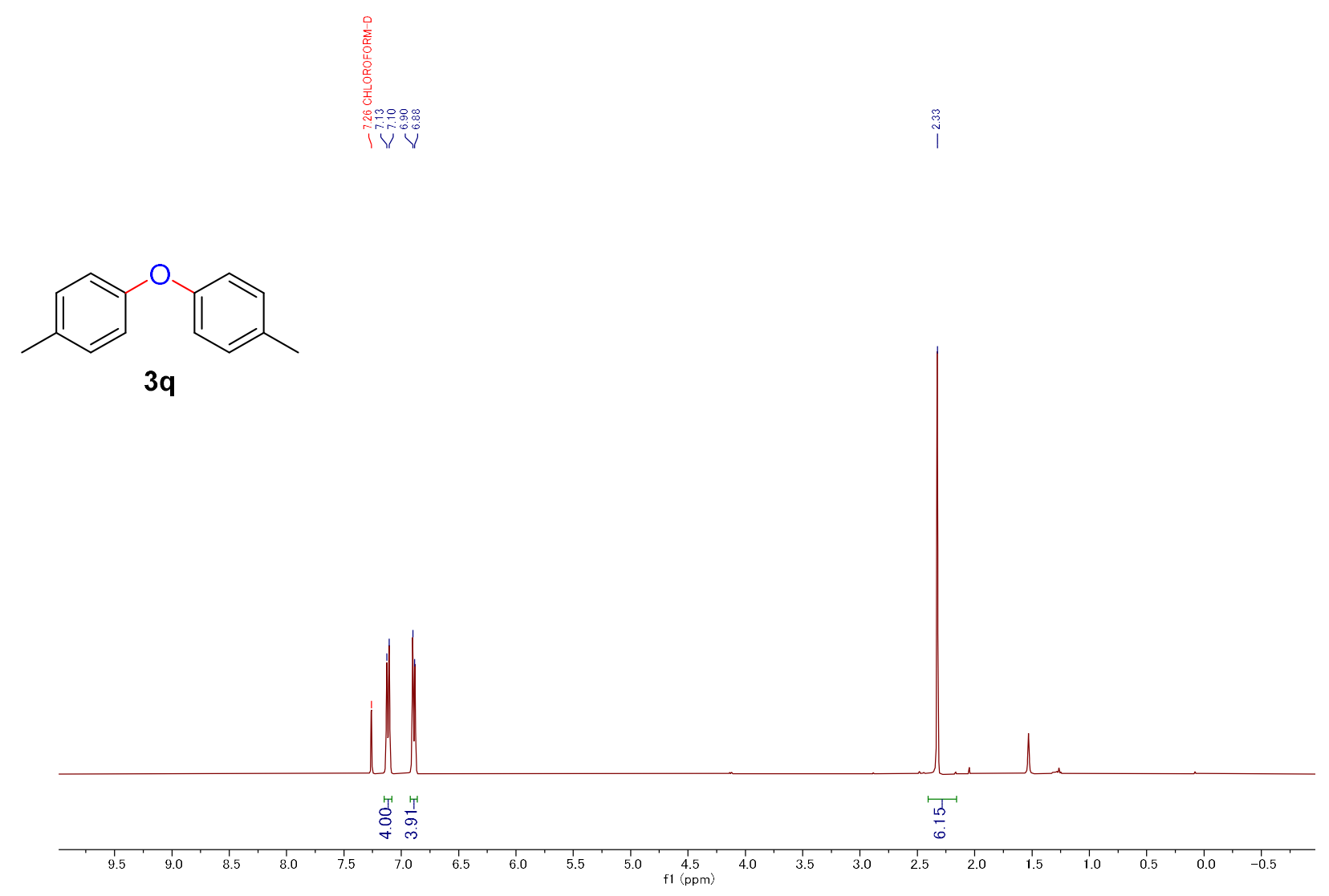

Figure S53. ${ }^{1} \mathrm{H} \mathrm{NMR}\left(400 \mathrm{MHz}, \mathrm{CDCl}_{3}\right)$ of 4,4'-dimethyldiphenyl ether (3q).
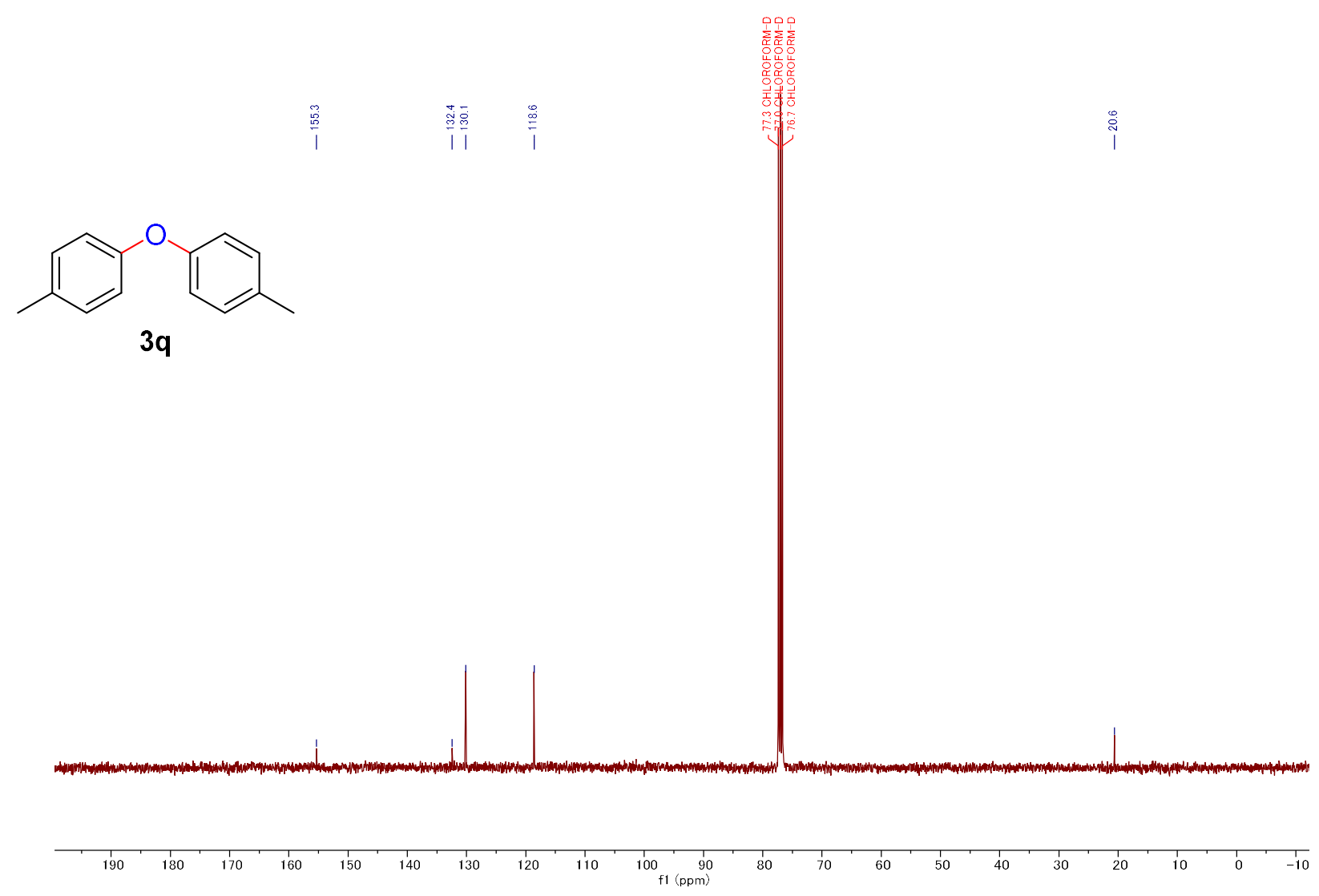

Figure S54. ${ }^{13} \mathrm{C}$ NMR (101 MHz, $\mathrm{CDCl}_{3}$ ) of 4,4'-dimethyldiphenyl ether (3q). 


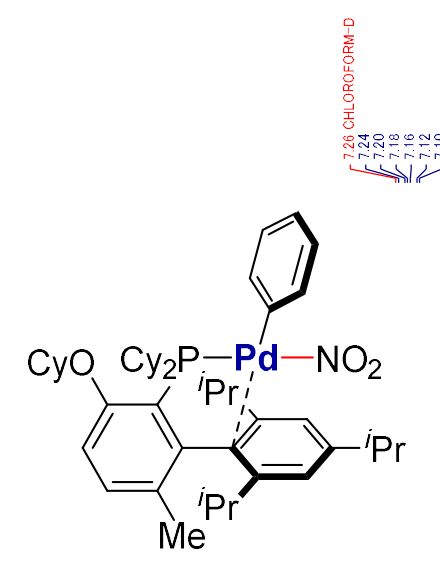

$4 a$

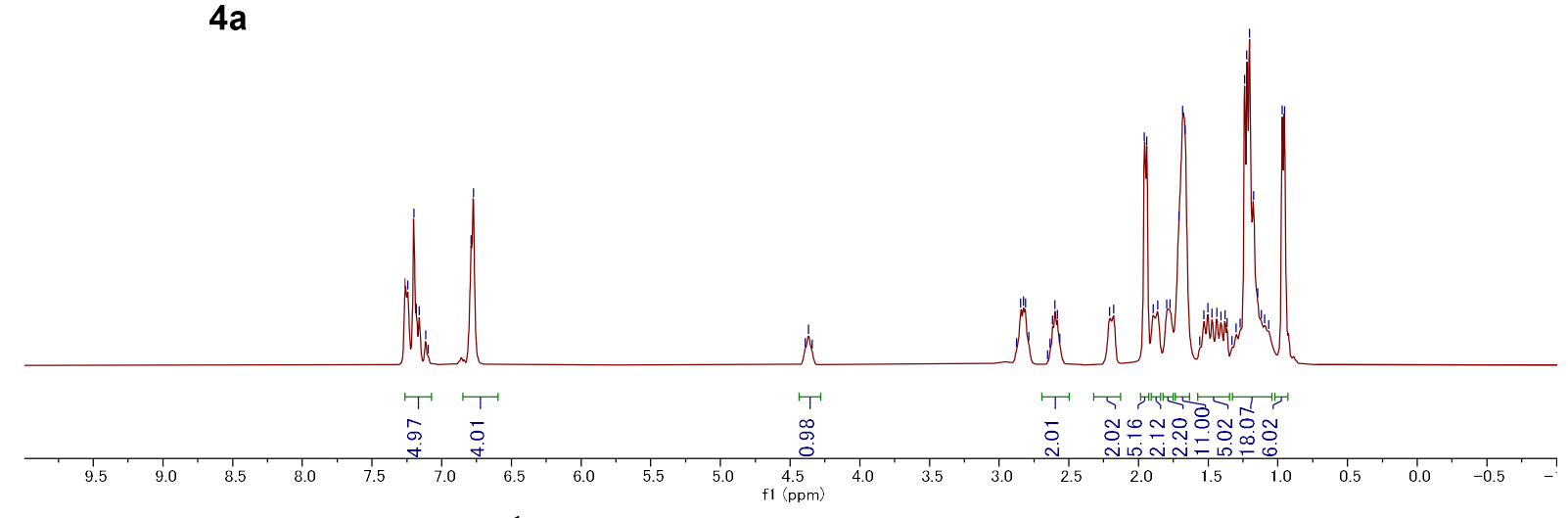

Figure S55. ${ }^{1} \mathrm{H}$ NMR $\left(400 \mathrm{MHz}, \mathrm{CDCl}_{3}\right)$ of $(\mathbf{L 9}) \mathrm{Pd}(\mathrm{Ph})\left(\mathrm{NO}_{2}\right)(\mathbf{4 a})$.

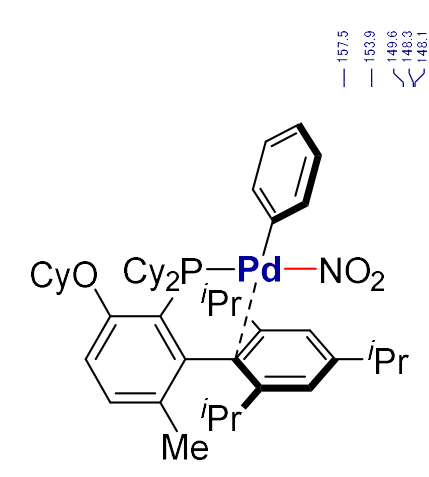

$4 a$

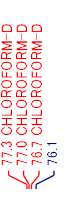

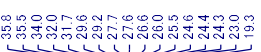

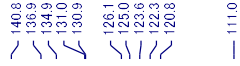

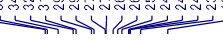

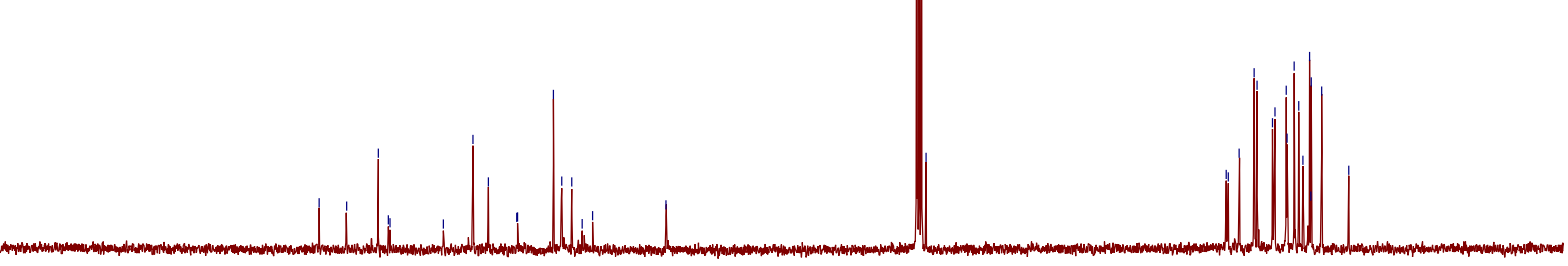

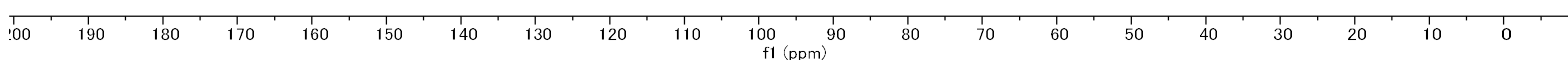

Figure S56. ${ }^{13} \mathrm{C}$ NMR (101 MHz, $\left.\mathrm{CDCl}_{3}\right)$ of $(\mathbf{L 9}) \mathrm{Pd}(\mathrm{Ph})\left(\mathrm{NO}_{2}\right)(\mathbf{4 a})$. 

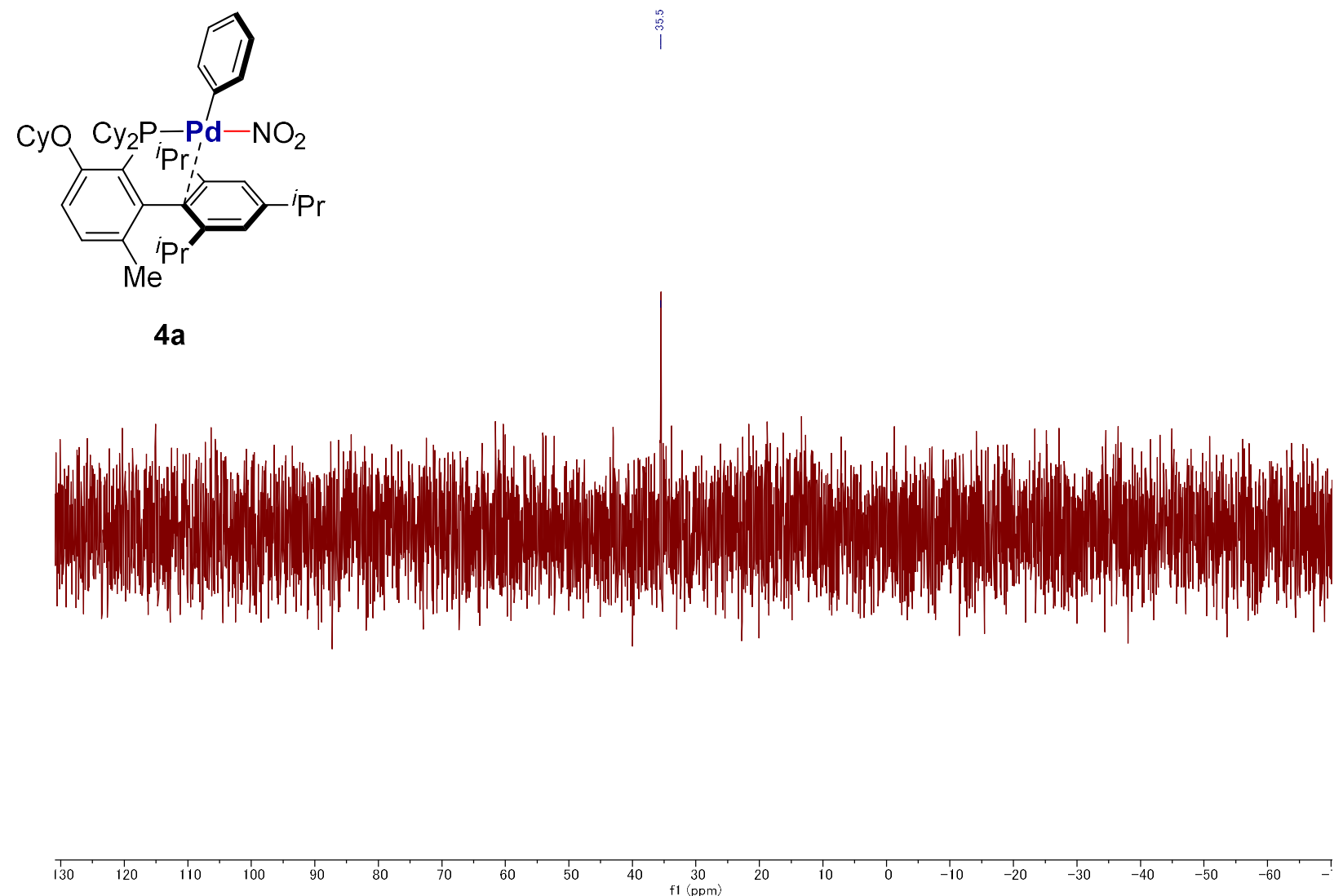

Figure S57. ${ }^{31} \mathrm{P}$ NMR $\left(162 \mathrm{MHz}, \mathrm{CDCl}_{3}\right)$ of $(\mathbf{L 9}) \mathrm{Pd}(\mathrm{Ph})\left(\mathrm{NO}_{2}\right)(\mathbf{4 a})$.

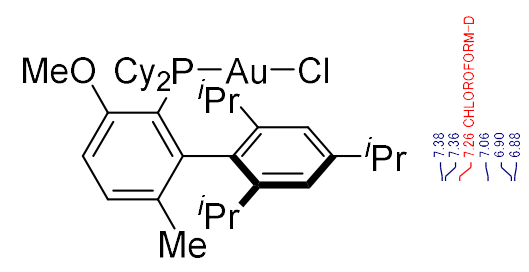

$6 b$
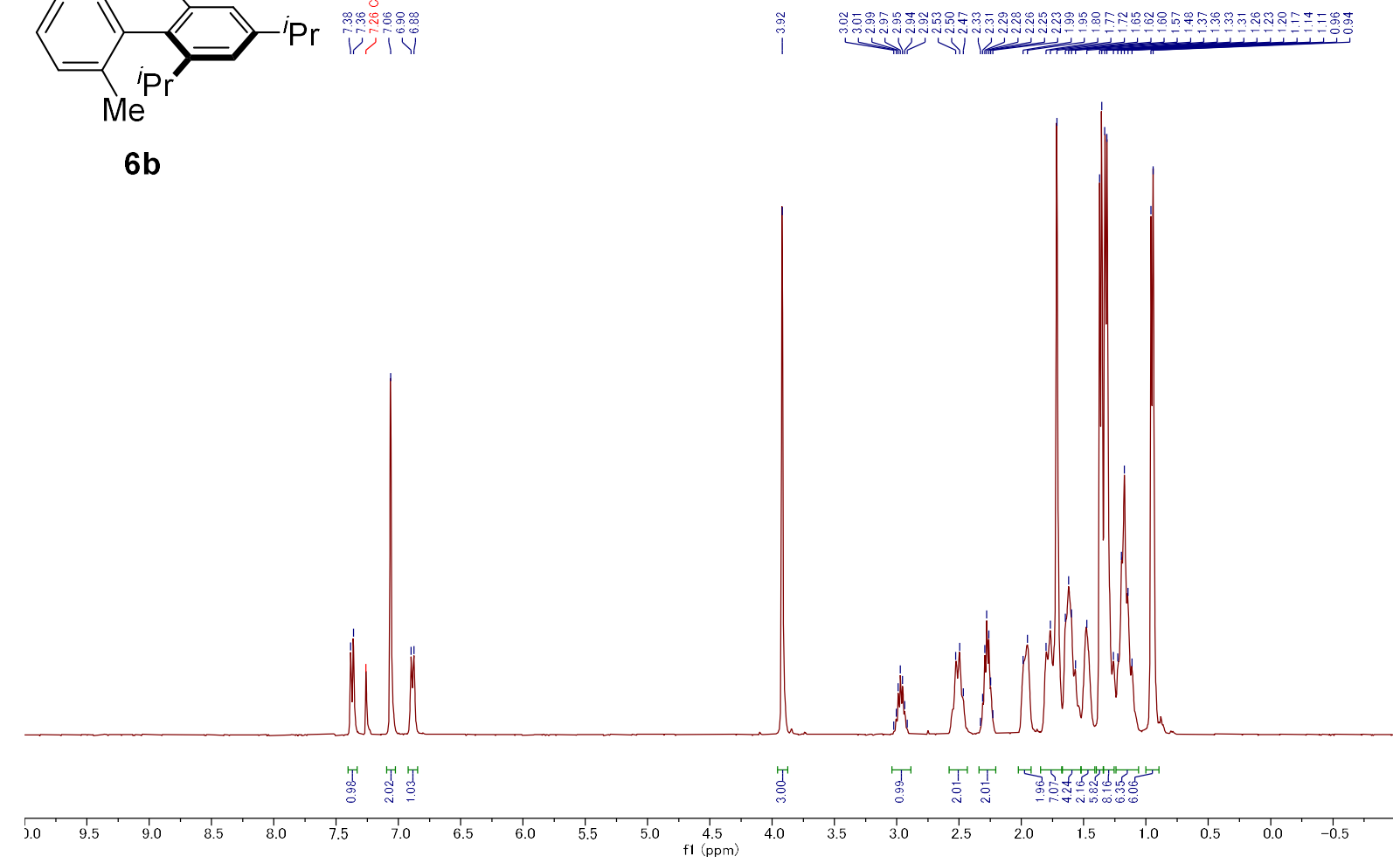

Figure S58. ${ }^{1} \mathrm{H}$ NMR (400 MHz, $\left.\mathrm{CDCl}_{3}\right)$ of (L5) $\mathrm{AuCl}(\mathbf{6 b})$. 

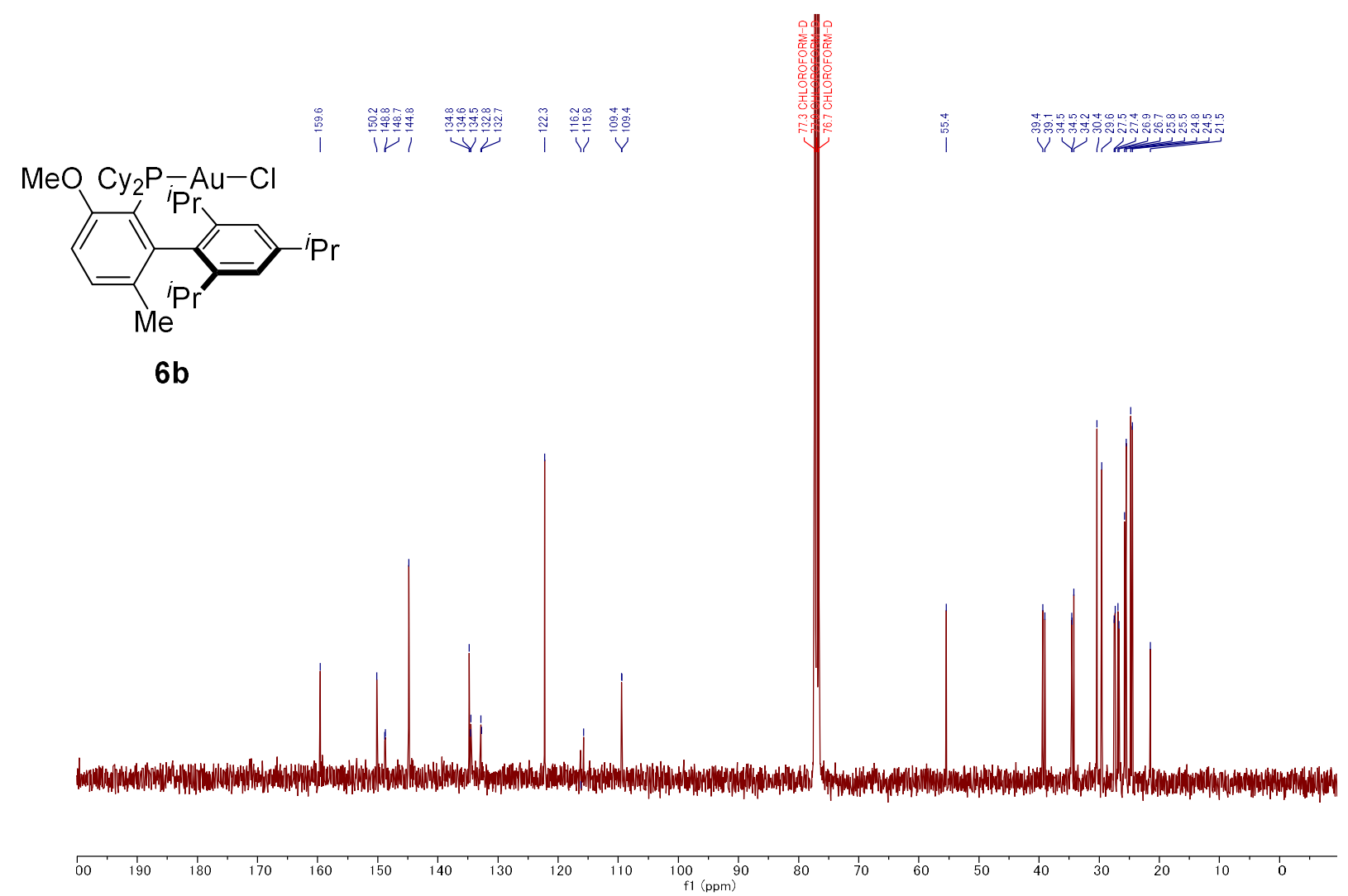

Figure S59. ${ }^{13} \mathrm{C} \mathrm{NMR}\left(101 \mathrm{MHz}, \mathrm{CDCl}_{3}\right)$ of (L5) $\mathrm{AuCl}(\mathbf{6 b})$.

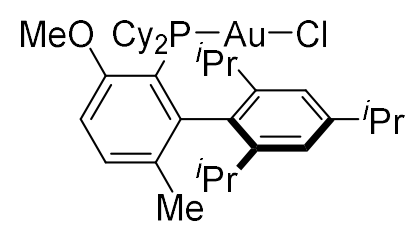

$6 b$

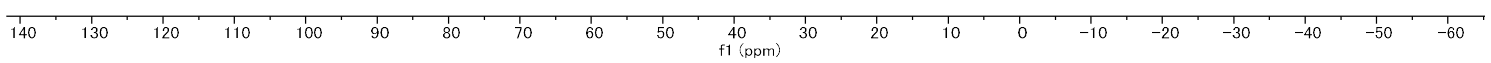

Figure S60. ${ }^{31} \mathrm{P} \mathrm{NMR}\left(162 \mathrm{MHz}, \mathrm{CDCl}_{3}\right)$ of (L5)AuCl (6b). 


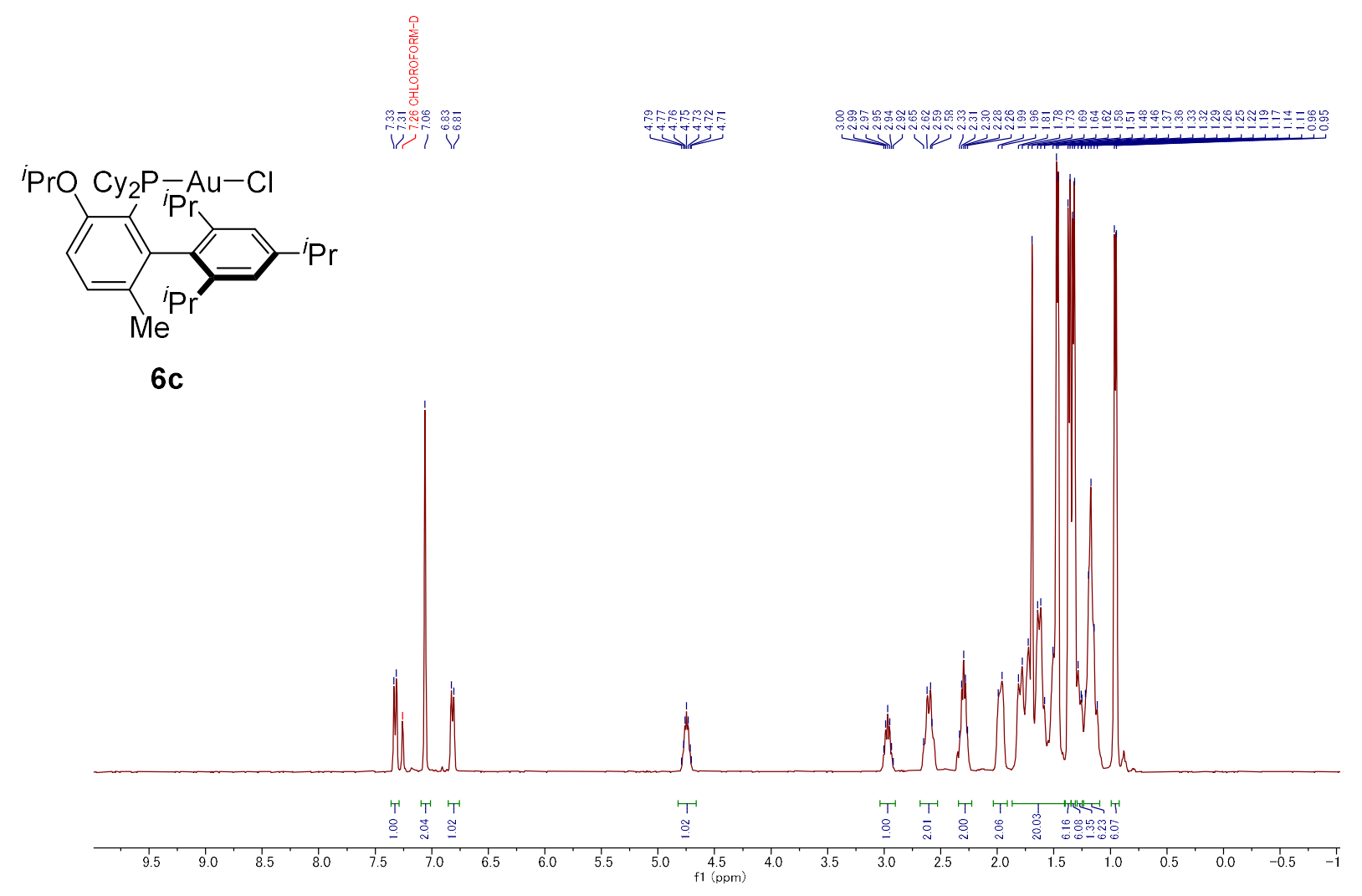

Figure S61. ${ }^{1} \mathrm{H} \mathrm{NMR}\left(400 \mathrm{MHz}, \mathrm{CDCl}_{3}\right)$ of $(\mathbf{L 7}) \mathrm{AuCl}(\mathbf{6 c})$
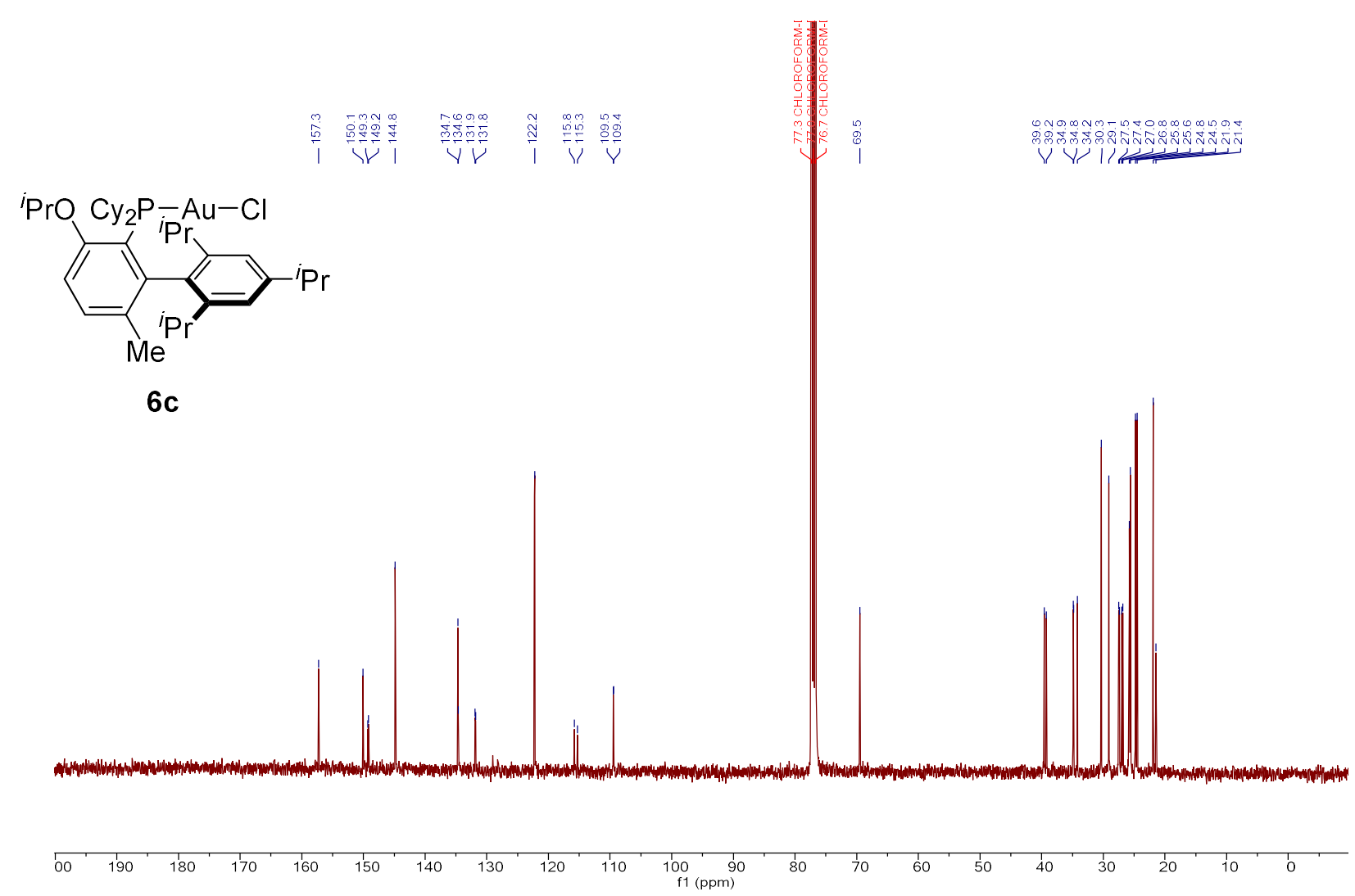

Figure S62. ${ }^{13} \mathrm{C} \mathrm{NMR}\left(101 \mathrm{MHz}, \mathrm{CDCl}_{3}\right)$ of $(\mathbf{L 7}) \mathrm{AuCl}(\mathbf{6 c})$. 


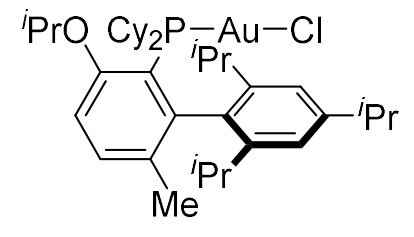

$6 c$

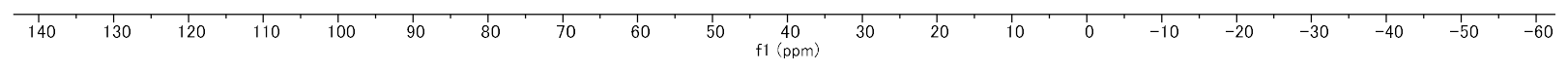

Figure S63. ${ }^{31} \mathrm{P}$ NMR $\left(162 \mathrm{MHz}, \mathrm{CDCl}_{3}\right)$ of (L7) $\mathrm{AuCl}(\mathbf{6 c})$.

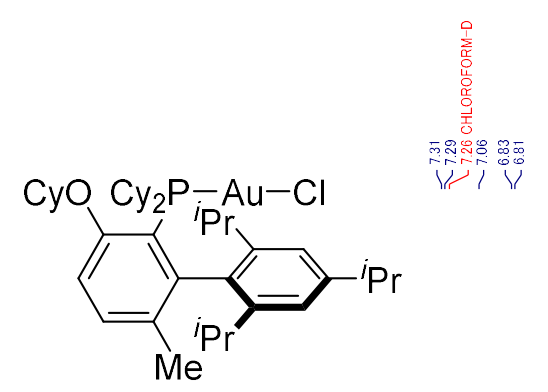

$6 d$

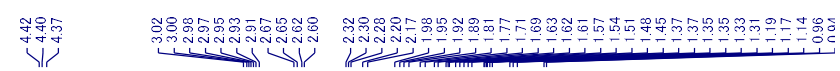

$\underbrace{-4}$
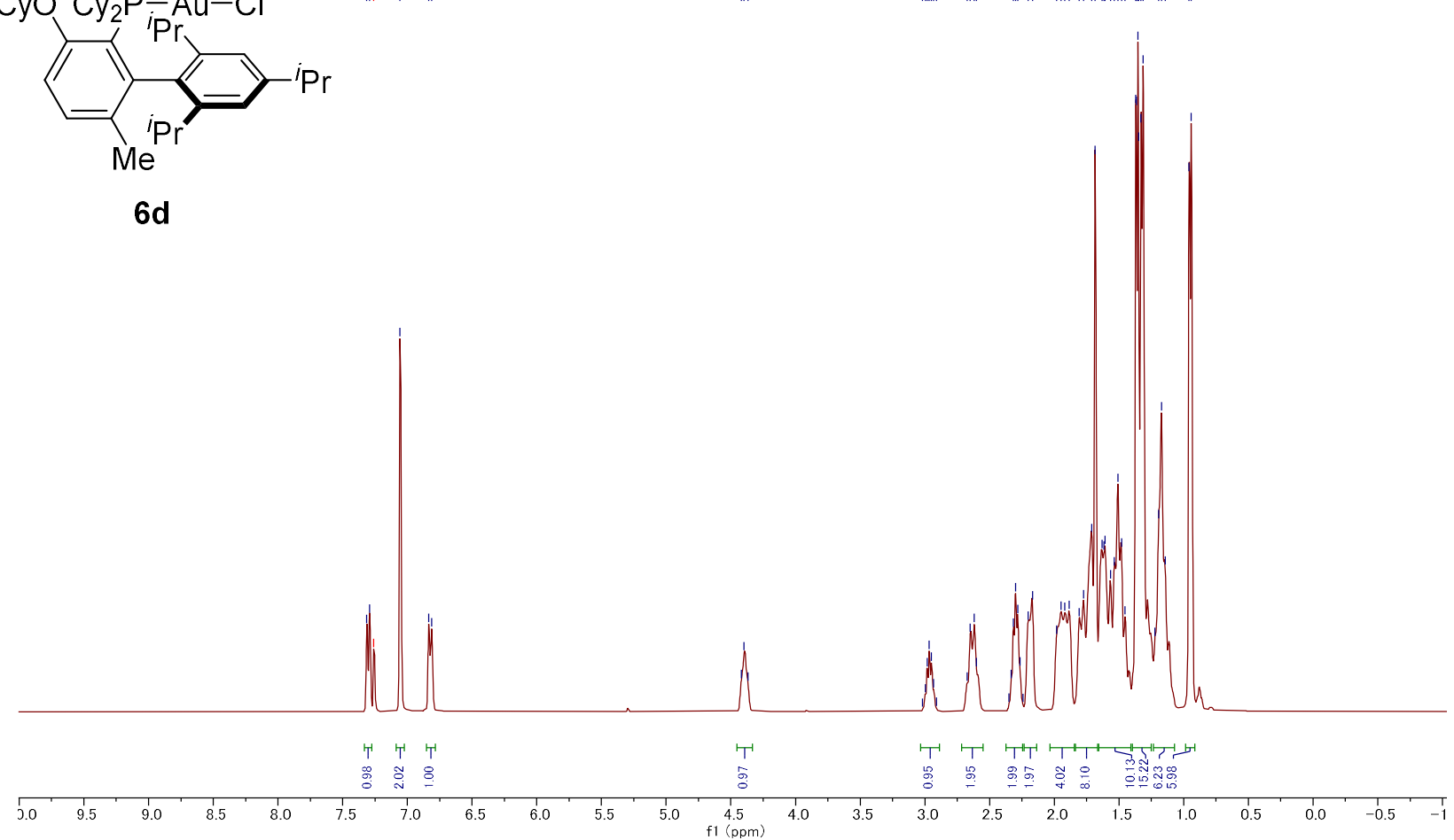

Figure S64. ${ }^{1} \mathrm{H}$ NMR (400 MHz, $\left.\mathrm{CDCl}_{3}\right)$ of (L9) $\mathrm{AuCl}(\mathbf{6 d})$. 

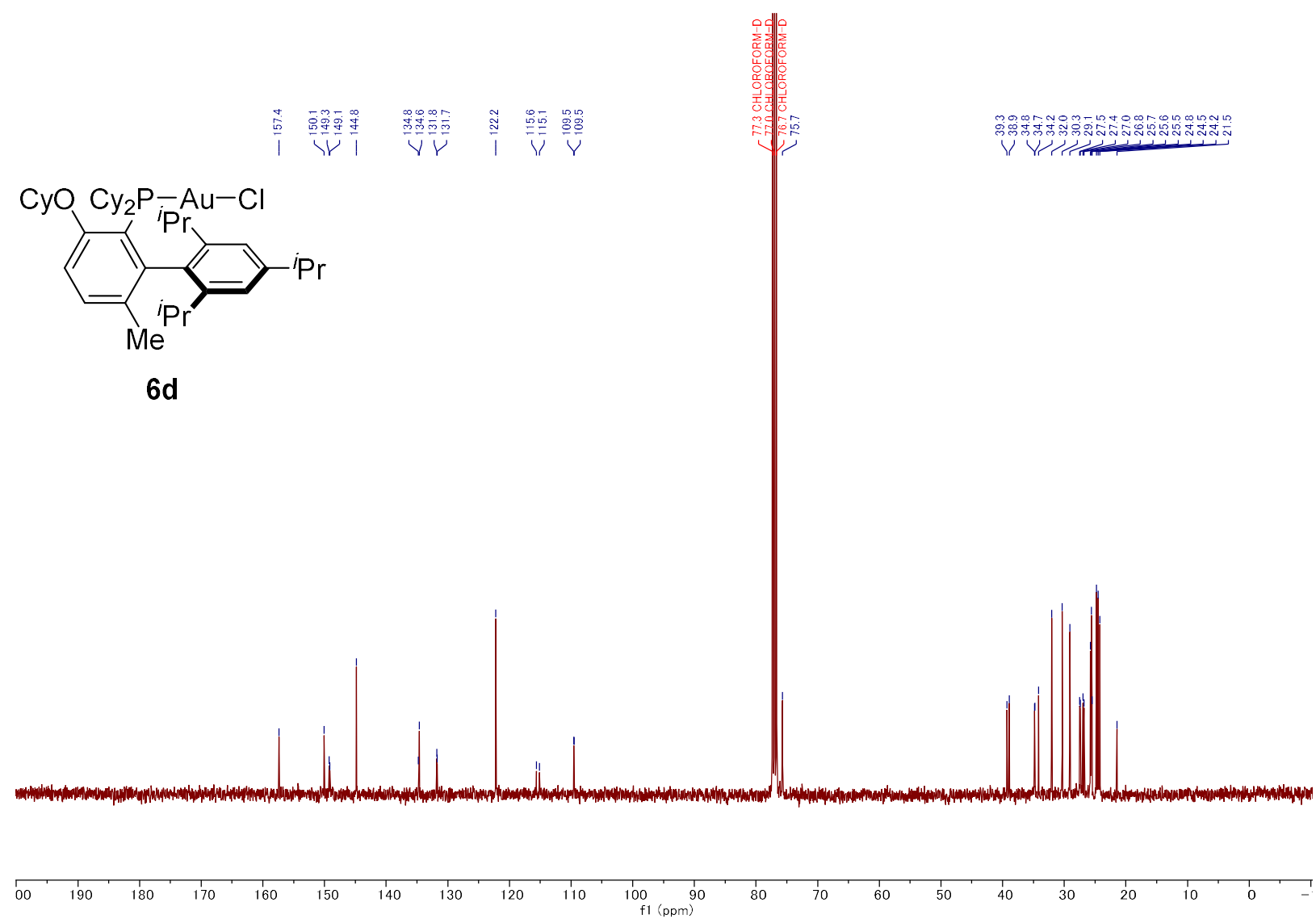

Figure S65. ${ }^{13} \mathrm{C} \mathrm{NMR}\left(101 \mathrm{MHz}, \mathrm{CDCl}_{3}\right)$ of (L9) $\mathrm{AuCl}(\mathbf{6 d})$.

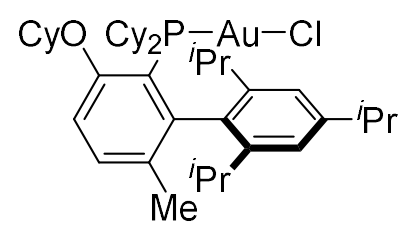

$6 d$

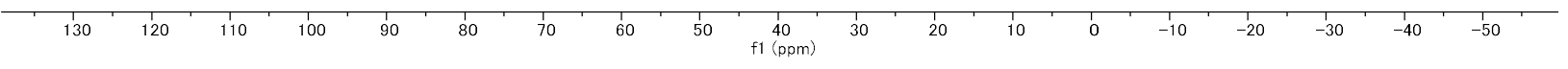

Figure S66. ${ }^{31} \mathrm{P} \mathrm{NMR}\left(162 \mathrm{MHz}, \mathrm{CDCl}_{3}\right)$ of (L9) $\mathrm{AuCl}(\mathbf{6 d})$. 


\section{References}

(1) Pangborn, A. B.; Giardello, M. A.; Grubbs, R. H.; Rosen, R. K.; Timmers, F. J. Safe and Convenient Procedure for Solvent Purification. Organometallics 1996, 15, 1518-1520.

(2) Olsen, E. P. K.; Arrechea, P. L.; Buchwald, S. L. Mechanistic Insight Leads to a Ligand Which Facilitates the Palladium-Catalyzed Formation of 2-(Hetero)Arylaminooxazoles and 4(Hetero)Arylaminothiazoles. Angew. Chem. Int. Ed. 2017, 56, 10569-10572.

(3) Wu, X.; Fors, B. P.; Buchwald, S. L. A Single Phosphine Ligand Allows Palladium-Catalyzed Intermolecular $\mathrm{C}-\mathrm{O}$ Bond Formation with Secondary and Primary Alcohols. Angew. Chem. Int. Ed. 2011, 50, 9943-9947.

(4) Kim, H. J.; Kim, M.; Chang, S. Rhodium(NHC)-Catalyzed $O$-Arylation of Aryl Bromides. Org. Lett. 2011, 13, 2368-2371.

(5) Naidu, A. B.; Jaseer, E. A.; Sekar, G. General, Mild, and Intermolecular Ullmann-Type Synthesis of Diaryl and Alkyl Aryl Ethers Catalyzed by Diol-Copper(I) Complex. J. Org. Chem. 2009, 74, 3675-3679.

(6) Chiang, G. C. H.; Olsson, T. Polymer-Supported Copper Complex for C-N and C-O CrossCoupling Reactions with Aryl Boronic Acids. Org. Lett. 2004, 6, 3079-3082.

(7) Zhang, Q.; Wang, D.; Wang, X.; Ding, K. (2-Pyridyl)Acetone-Promoted Cu-Catalyzed $O$ Arylation of Phenols with Aryl Iodides, Bromides, and Chlorides. J. Org. Chem. 2009, 74, 7187-7190.

(8) Mino, T.; Yagishita, F.; Shibuya, M.; Kajiwara, K.; Shindo, H.; Sakamoto, M.; Fujita, T. Copper(I)-Catalyzed C-C and C-O Coupling Reactions Using Hydrazone Ligands. Synlett 2009, 2457-2460.

(9) Tlili, A.; Monnier, F.; Taillefer, M. Selective One-Pot Access to Symmetrical or Unsymmetrical Diaryl Ethers by Copper-Catalyzed Double Arylation of a Simple Oxygen Source. Chem. Eur. J. 2010, 16, 12299-12302.

(10) Xing, L.; Wang, X.; Cheng, C.; Zhu, R.; Liu, B.; Hu, Y. A Solvent-Controlled Highly Efficient $\mathrm{Pd}-\mathrm{C}$ Catalyzed Hydrogenolysis of Benzaldehydes to Methylbenzenes via a Novel "Acetal Pathway." Tetrahedron 2007, 63, 9382-9386.

(11) Yang, K.; Li, Z.; Wang, Z.; Yao, Z.; Jiang, S. Highly Efficient Synthesis of Phenols by CopperCatalyzed Hydroxylation of Aryl Iodides, Bromides, and Chlorides. Org. Lett. 2011, 13, 4340 4343.

(12) Rigaku Corporation, 1999; CrystalClear Software User's Guide, Molecular Structure Corporation, 2000.

(13) Pflugrath, J. W. The finer things in X-ray diffraction data collection. Acta Crystallogr. Sect. D Biol. Crystallogr. 1999, 55, 1718-1725.

(14) Sheldrick, G. M. SHELXT - Integrated Space-Group and Crystal-Structure Determination. Acta Crystallogr. Sect. A Found. Crystallogr. 2015, 71, 3-8.

(15) Sheldrick, G. M. Crystal Structure Refinement with SHELXL. Acta Crystallogr. Sect. C Struct. Chem. 2015, 71, 3-8.

(16) Altomare, A. Cascarano, G. Giacovazzo, C. Guagliardi, A. Completion and refinement of crystal structures with SIR92. J. Appl. Cryst. 1993, 26, 343-350.

(17) Sheldrick, G. M. A short history of SHELX. Acta Crystallogr. Sect. A Found. Crystallogr. 2008, $64,112-122$.

(18) (a) Becke, A. D. Density-functional exchange-energy approximation with correct asymptotic behavior. Phys. Rev. A 1988, 38, 3098-3100. (b) Perdew, J. P.; Wang, Y. Accurate and simple analytic representation of the electron-gas correlation energy. Phys. Rev. B 1992, 45, 13244 13249. (c) Becke, A. D. Density-functional thermochemistry. III. The role of exact exchange. J. Chem. Phys. 1993, 98, 5648-5652. (d) Perdew, J. P.; Burke, K.; Wang, Y. Generalized gradient approximation for the exchange-correlation hole of a many-electron system. Phys. Rev. $B$ 1996, 54, 16533-16539. (e) Perdew, J. P. In Electronic Structure of Solids, 91th ed.; Ziesche, P.; Eschrig, H. Eds.; Akademie Verlag: Berlin, 1991. (f) Burke, K.; Perdew, J. P.; Wang, Y. In 
Electronic Density Functional Theory: Recent Progress and New Directions; Dobson, J. F.; Vignale, G.; Das, M. P. Eds.; Plenum: New York, 1998.

(19) (a) Grimme, S.; Ehrlich, S.; Goerigk, L. Effect of Damping Function in Dispersion Corrected Density Functional Theory J. Comput. Chem. 2011, 32, 1456-1465. (b) Grimme, S.; Antony, J.; Ehrlich, S.; Krieg, H. A. A consistent and accurate $a b$ initio parametrization of density functional dispersion correction (DFT-D) for the 94 elements $\mathrm{H}-\mathrm{Pu} . J$. Chem. Phys. 2010, 132, 154104-154119.

(20) Hay, P.; Jeffrey, W. W. R. Ab initio effective core potentials for molecular calculations. Potentials for K to Au including the outermost core orbitals. J. Chem. Phys. 1985, 82, 299-310.

(21) (a) Ditchfield, R.; Hehre, W. J.; Pople, J. A. Self-Consistent Molecular-Orbital Methods. IX. An Extended Gaussian-Type Basis for Molecular-Orbital Studies of Organic Molecules. J. Chem. Phys. 1971, 54, 724-728. (b) Francl, M. M.; Pietro, W. J.; Hehre, W. J.; Binkley, J.; Stephen, G. M. S.; DeFrees, D. J.; Pople, J. A. Self-consistent molecular orbital methods. XXIII. A polarization-type basis set for second-row elements. J. Chem. Phys. 1982, 77, 3654-3665. (c) Gordon, M. S.; Binkley, J.; Stephen, P, J. A.; Pietro, W. J.; Hehre, W. J. Self-consistent molecular-orbital methods. 22. Small split-valence basis sets for second-row elements. J. Am. Chem. Soc. 1982, 104, 2797-2803. (d) Hariharan, P. C.; Pople, J. A. The influence of polarization functions on molecular orbital hydrogenation energies. Theor. Chim. Acta 1973, 28, 213-222. (e) Hehre, W. J.; Ditchfield, R.; Pople, J. A. Self-Consistent Molecular Orbital Methods. XII. Further Extensions of Gaussian-Type Basis Sets for Use in Molecular Orbital Studies of Organic Molecules. J. Chem. Phys. 1972, 56, 2257-2261.

(22) Frisch, M. J.; Trucks, G. W.; Schlegel, H. B.; Scuseria, G. E.; Robb, M. A.; Cheeseman, J. R.; Scalmani, G.; Barone, V.; Petersson, A.; Nakatsuji, H.; Li, X.; Caricato, M.; Marenich, M. V.; Bloino, J.; Janesko, B. G.; Gomperts, R.; Mennucci, B.; Hratchian, H. P.; Ortiz, J. V.; Izmaylov, A. F.; Sonnenberg, J. L.; Williams-Young, D.; Ding, F.; Lipparini, F.; Egidi, F.; Goings, J.; Peng, B.; Petrone, A.; Henderson, T.; Ranasinghe, D.; Zakrzewski, V. G.; Gao, J.; Rega, N.; Zheng, G.; Liang, W.; Hada, M.; Ehara, M.; Toyota, K.; Fukuda, R.; Hasegawa, J.; Ishida, M.; Nakajima, T.; Honda, T.; Kitao, O.; Nakai, H.; Vreven, T.; Throssell, K.; Montgomery, J. A.; Peralta, Jr. J. E.; Ogliaro, F.; Bearpark, M. J.; Heyd, J. J.; Brothers, E. N.; Kudin, K. N.; Staroverov, V. N.; Keith, T. A.; Kobayashi, R.; Normand, J.; Raghavachari, K.; Rendell, A. P.; Burant, J. C.; Iyengar, S. S.; Tomasi, J.; Cossi, M.; Millam, J. M.; Klene, M.; Adamo, C.; Cammi, R.; Ochterski, J. W.; Martin, R. L.; Morokuma, K.; Farkas, O.; Foresman, J. B.; Fox, D. J. Gaussian 16, revision B.01; Gaussian, Inc.: Wallingford, CT, 2016. 